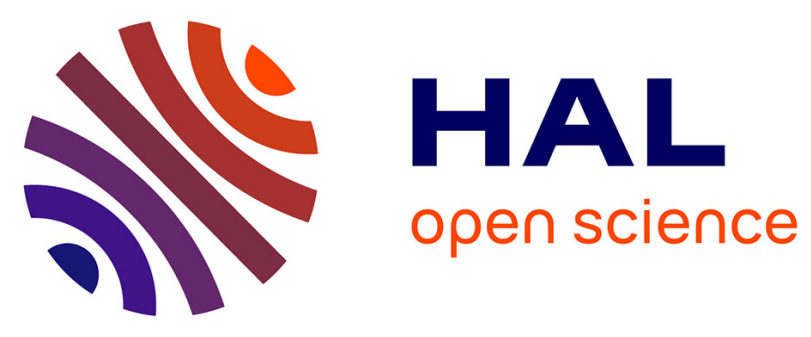

\title{
Experimental studies on the instantaneous fluid-structure interaction of an air-inflated flexible membrane in turbulent flow
}

\author{
Jens Nikolas Wood, Michael Breuer, Guillaume de Nayer
}

\section{To cite this version:}

Jens Nikolas Wood, Michael Breuer, Guillaume de Nayer. Experimental studies on the instantaneous fluid-structure interaction of an air-inflated flexible membrane in turbulent flow. Journal of Fluids and Structures, 2018, 80, pp.405 - 440. 10.1016/j.jfluidstructs.2018.02.006 . hal-01861431

\author{
HAL Id: hal-01861431 \\ https://hal.science/hal-01861431
}

Submitted on 27 Aug 2018

HAL is a multi-disciplinary open access archive for the deposit and dissemination of scientific research documents, whether they are published or not. The documents may come from teaching and research institutions in France or abroad, or from public or private research centers.
L'archive ouverte pluridisciplinaire HAL, est destinée au dépôt et à la diffusion de documents scientifiques de niveau recherche, publiés ou non, émanant des établissements d'enseignement et de recherche français ou étrangers, des laboratoires publics ou privés.

\section{(2)(1) $\$$}

Distributed under a Creative Commons Attribution - NonCommercial - NoDerivatives $\mid 4.0$ 


\title{
Experimental Studies on the Instantaneous Fluid-Structure Interaction of an Air-Inflated Flexible Membrane in Turbulent Flow
}

\author{
J. N. Wood, M. Breuer*, G. De Nayer \\ Professur für Strömungsmechanik, Helmut-Schmidt-Universität Hamburg, D-22043 Hamburg, Germany
}

\begin{abstract}
The present paper investigates the interaction between a turbulent fluid flow and a flexible membrane structure. Such flexible structures are of increasing interest for modern engineering applications due to their adaptable utilization. Highly flexible membranes under turbulent flow conditions still bare fundamental challenges such as the structural response to fluid loads leading to the motivation of the present study. It investigates the fluid-structure interaction of a flexible membranous structure in the shape of a hemisphere. The air-inflated structure is placed in the test section of a wind tunnel and is exposed to a turbulent boundary layer flow. The properties of the turbulent boundary layer are clearly defined so that the test case is reproducible by numerical simulations. Three Reynolds numbers (50,000, 75,000 and 100,000) are chosen to examine the interaction between the turbulent flow and the pressurized membrane. Special emphasis is put on the instantaneous effects. Furthermore, the flow field around an equally sized rigid hemisphere is measured under identical conditions serving as a reference for the flexible case. The experiments are conducted by combining particle-image-velocimetry for the flow field and high-speed digital-image correlation measurements for the deformation of the oscillating membrane. Furthermore, a constant-temperature anemometer is used for evaluating the velocity spectra at locations close to the wall to connect the independently performed fluid and structure measurements. A thorough analysis of the comprehensive data sets for the fluid flow and the displacements of the structure leads to the characterization of the behavior of the flexible structure under changing flow conditions.
\end{abstract}

Keywords: fluid-structure interaction; hemisphere; membrane; turbulent flow; particle-image-velocimetry; digital-image correlation; fluid load

\section{Introduction}

Flexible structures interacting with fluid flows are fundamental physical phenomena omnipresent in nature and technical applications. A deeper understanding of the general characteristics of coupled systems is of common interest. Thus, multi-physical approaches are developed and applied to investigate fluid-structure interaction (FSI) problems in various fields of science.

In modern engineering thin flexible structures are essential construction elements. . Thin and shapeable materials are often taken into account when weight reduction, space-saving and individualized design are major objectives of a project. These requirements are often satisfied by membrane structures that feature a cost-efficient solution for various applications . As an example Fig. 1(a) illustrates typical shapes of membranes used as main structural components

*Corresponding author

Email address: breuer@hsu-hh.de (M. Breuer)

(C)2018. This manuscript version is made available under the CC-BY-NC-ND 4.0 license

http://creativecommons.org/licenses/by-nc-nd/4.0/ 
for light-weight buildings. A few examples of membrane applications are selected from the literature to outline their operation spectrum.

First fundamental studies on inflated spherically shaped structures under wind loads were carried out by Newman et al. (1984). The study targeted at a proposal on the safe operation of air-inflated buildings exposed to wind. A critical parameter for this is the gauge pressure inside such buildings keeping the inflated structure stable. The research was divided into two sections: First, the flow around three rigid domes with different height-to-base-radius ratios $(h /(D / 2)=$ $0.5,0.37$ and 0.25$)$ made of Plexiglas were measured in a boundary layer wind tunnel mainly focusing on the surface pressure distribution and smoke visualization of the flow patterns at $\operatorname{Re}=50,800$, based on the base diameter of the domed structure. The results of the pressure measurements were afterwards transferred to a numerical simulation based on a finite-element solver in order to obtain the stresses in the plane of the membrane. The simulation was used to predict the position and orientation of first buckling onset while reducing the gauge pressure step-wise. To evaluate the numerical results obtained, the rigid models were exchanged by inflatable models made of impervious, effectively unstretchable light cloth. After reducing the inner pressure during wind tunnel operation, it was possible to observe the deformations on each flexible model. Based on this experiment, the critical gauge pressure was estimated to be about 0.65 times the dynamic pressure measured at the apex of the dome. Dynamic effects of the wind load excitations of the flexible structure were not taken into account.

Gong et al. (2010) presented the further utilization of the China National Stadium which was built for the 29th Olympic Games in Beijing. An air-inflated membrane is suggested to cover the open roof construction of the stadium in order to protect the inside from external factors like rain or extensive sun. In general, inflated membranous structures can be used as permanent or temporary installations. A model at a scale of 1:20 was manufactured to validate analytic results by experimental measurements. After testing the mechanical behavior of the inflatable structure by different static loads, the overall construction seems to be suitable for the intended purpose. However, unsteady wind loads are not taken into account because the overall size of the model $(9.12 \mathrm{~m} \times 6.2 \mathrm{~m} \times 1.362 \mathrm{~m})$ is difficult to transfer to a suitable test facility.

Another inflatable application is a portable ultra light-weight emergency shelter conducted by the "uLites" research program ${ }^{1}$. The main design feature focuses on the fast assembly of temporary housings in regions that are exposed to natural disasters, where a quick deployment of adequate first supply is mandatory. The construction consists of air-inflatable arc-shaped membranous tubes that can be connected to a conjoint module of desired length. For the fast accessibility, the hangar-like building is open at both sides. This makes it vulnerable to unsteady wind loads that can cause high lift forces. To estimate this load, wind tunnel experiments with down-scaled models were conducted by Larese et al. (2014a,b). In parallel, a virtual wind tunnel for advanced coupled simulations of membrane systems was implemented by Rossi (2013) to validate the experimental data and to reduce the costs of large-scale tests. Similarly, Ligaro and Barsotti (2013) presented a numerical study of a thin hemispherical dome which is composed of a lattice of interacting inflatable beams and panels. The internal pressure of the inflated beams induce a pre-stress in the panels stabilizing the dome. This case differs from an ordinary inflated structure, where the whole inner domain of the building is pressurized. One advantage of inflated beams is the weight reduction in comparison to standard metallic elements. Several load cases, such as a static wind load approximated by the guidelines of

\footnotetext{
${ }^{1}$ http://www. cimne.com/websasp/ulites/default.asp

${ }^{2}$ http://www .architen.com/wp-content/uploads/2009/03/basic-theories-image-2.jpg

${ }^{3}$ http://www.ketchum.org/shellpix.html
} 

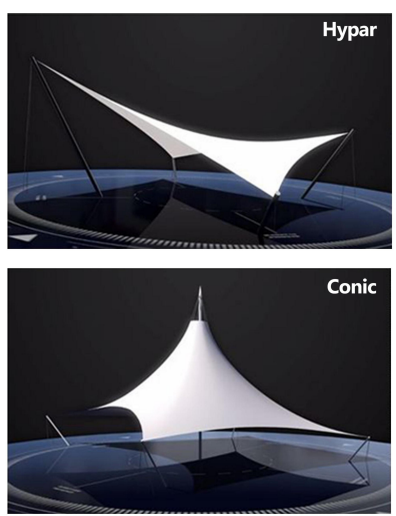

(a) Possible shapes of membranous structures ${ }^{2}$.
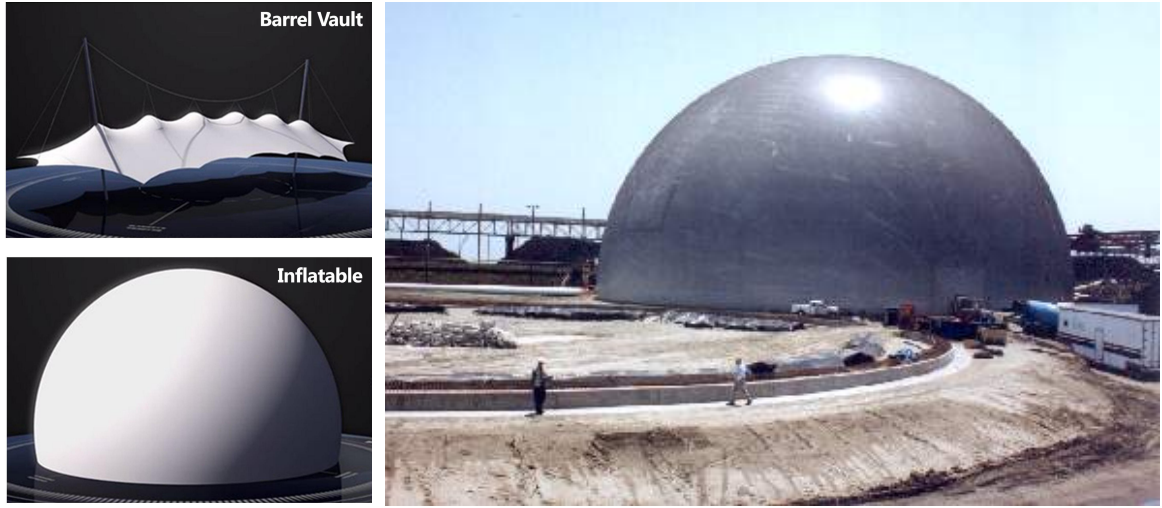

(b) Air-inflated hemisphere (Los Angeles) $)^{3}$.

Figure 1: Examples of flexible membranous structures.

the Italian NTC2008 norm (Norm Tecniche Costruzioni 2008), were applied to examine the mechanical behavior of the structure. It is claimed that the composite of inflated beams and panels is less vulnerable to incidental damage than standard fully inflated structures.

A detailed experimental investigation of the unsteady aeroelastic mechanism of pre-tensioned membrane structures exposed to uniform flow was carried out by $\mathrm{Wu}$ et al. (2015). The wind tunnel models were saddle-shaped with a rigid primary structure and a flat membranous top made of a $0.4 \mathrm{~mm}$ thin latex sheet. Laser displacement sensors tracked the deformation of the membrane at certain surface points, while the structure was excited by unsteady flow conditions. The flow field was recorded by a hot-wire probe. Detailed analyses of the frequency response of the flexible structure were presented and set into correlation with the flow field measurements. It was observed that the on-coming wind load deforms the membranous roof which results in a quasi-static deflection. Superimposed to this deflection the structure vibrated in several modes. The amplitude of the excitation depends on the flow velocity. Under particular wind directions and within certain velocity ranges a lock-in between the structural excitation of the membrane and the vortex shedding was observed. This finding indicates the presence of a vortex-induced vibration (VIV) (Naudascher and Rockwell, 1994). The amplitude of the structure deflections dramatically increases under these conditions and can cause severe damage to the structure.

An elaborate study on aeroelasticity was carried out by Rojratsirikul et al. (2009) and Rojratsirikul et al. (2010) using a thin rubber sheet made of black latex with a thickness of $0.2 \mathrm{~mm}$ to mimic the characteristics of membrane wings. Flying mammals, such as bats, exhibit excellent maneuverability at low Reynolds numbers while hunting for insects. This ability is connected to the rapid adaption of the flexible wing to the particular flight condition. To study this phenomenon, measurements of the unsteady membrane shape were carried out using high-speed cameras with complementary measurements of the flow field applying particle-image-velocimetry in a wind tunnel at $\operatorname{Re}=53,100,79,700$ and 106,000, based on the chord length of the wing. The flow-induced vibrations of the flexible wing were analyzed in detail showing several well distinguishable modal shapes excited at different combinations of approaching flow velocity and angle of attack. These results were afterwards compared to a rigid wing showing significantly different flow characteristics. The Reynolds stresses on the upper side of the flexible wing revealed higher velocity fluctuations in the shear layer caused by the excitation of the rubber wing leading to the roll-up of large vortices. It was suggested that the flexible wing decreases the drag and delays the stall and is superior to the rigid case.

An early comprehensive experimental and numerical study was conducted by Rank et al. (2003) and Glück et al. (2003). The behavior of a large cable supported membranous shield was tested 
in a wind tunnel. Measurements concentrated on the forces acting on the retention cables and the deflection of the structure due to the wind loads. The results were compared with complementarily conducted coupled simulations based on RANS and showed good agreement concerning steady wind loads and displacements. Unsteady wind loads were not taken into account.

Similar to this study Michalski et al. (2011, 2015) performed experiments and numerical simulations of an umbrella-type structure in a turbulent boundary layer flow as typically found in urban regions. The large-scale experiments were conducted with a prototype of the dimensions $29 \mathrm{~m} \times 29 \mathrm{~m} \times 19 \mathrm{~m}(l \times w \times h)$ including detailed measurements of the unsteady wind profile at the test site. The dynamic response of the structure induced by the wind loads was measured by four cameras focusing on specific monitoring points at the tip of the umbrella recording the instantaneous deflections. Furthermore, the dynamic loads were measured by applying strain gauge elements (DMS technology) on the struts of the umbrella structure. The results were then compared with complementary numerical simulations. For this purpose, the statistical results of the experimental flow field were applied as inflow conditions for the numerical simulations based on LES and a finite-element solver. The results of the experiment and the numerical simulation were found to be in good agreement.

According to the applications mentioned above, the incorporation of membranes as main structural elements raise important design issues that have to be considered carefully. A major challenge in urban environments is the response of the flexible structure to instantaneous wind loads. Critical flow velocities or temporal gusts can trigger structural instabilities due to high static compression loads or dynamic response phenomena, such as vortex or movement induced excitations (MIE) (Naudascher and Rockwell, 1994).

This brief literature review leads to the motivation of the present paper. It was shown that the topic of fluid-structure interaction of membranous structures especially under instantaneous wind loads is of high relevance in a variety of civil engineering and aeroelastic applications. However, there is still a lack of fundamental experimental data, which take both the fluid and the structure side equivalently into account. Since such a data base is highly desirable for a better physical understanding but also for validation purposes of developed FSI simulation methodologies, the present study contributes to fill this gap. For this purpose, this experimental investigation focuses on a thin-walled air-inflated membranous structure in the shape of a hemisphere comparable to the air-inflated structure depicted in Fig. 1(b). This geometrically simple hemispherical dome is considered to be exposed to a thick turbulent boundary layer flow. The main objective is to generate reproducible and clearly defined boundary conditions, which can be applied in the complementary numerical simulations. This procedure allows a direct comparison with the predictions as required for a benchmark case of FSI for flexible structures. The experimental investigations are carried out in a subsonic wind tunnel including comprehensive particle-image-velocimetry (PIV) measurements of the flow field and digital-image correlation (DIC) measurements of the structural deformations. Furthermore, constant-temperature anemometry measurements are performed for specific locations close to the wall in order to determine the frequency spectrum in the wake flow. That allows to characterize the fluid-structure interaction between the deformable structure and the flow field. To identify the characteristics of the FSI phenomenon, the results are compared with the flow field investigations of a rigid hemisphere at Reynolds numbers of $\operatorname{Re}=\rho_{\text {air }} D U_{\infty} / \mu_{\text {air }}=50,000$, 75,000 and 100,000, ( $D$ is the diameter of the hemisphere). Characteristics of the flow field (frequency spectra and time-averaged data) and the response of the flexible structure are brought into correlation to determine the underlying fluid-structure interaction phenomena. 


\section{Experimental setup}

\subsection{Description of the physical case}

Figure 2 depicts the investigated case of a flexible hemisphere (diameter $D$ ) mounted on a smooth surface under turbulent flow conditions. In order to be reproducible by numerical simulations the thick turbulent boundary layer is composed of reasonable fluctuations superimposed on a classical turbulent velocity profile.

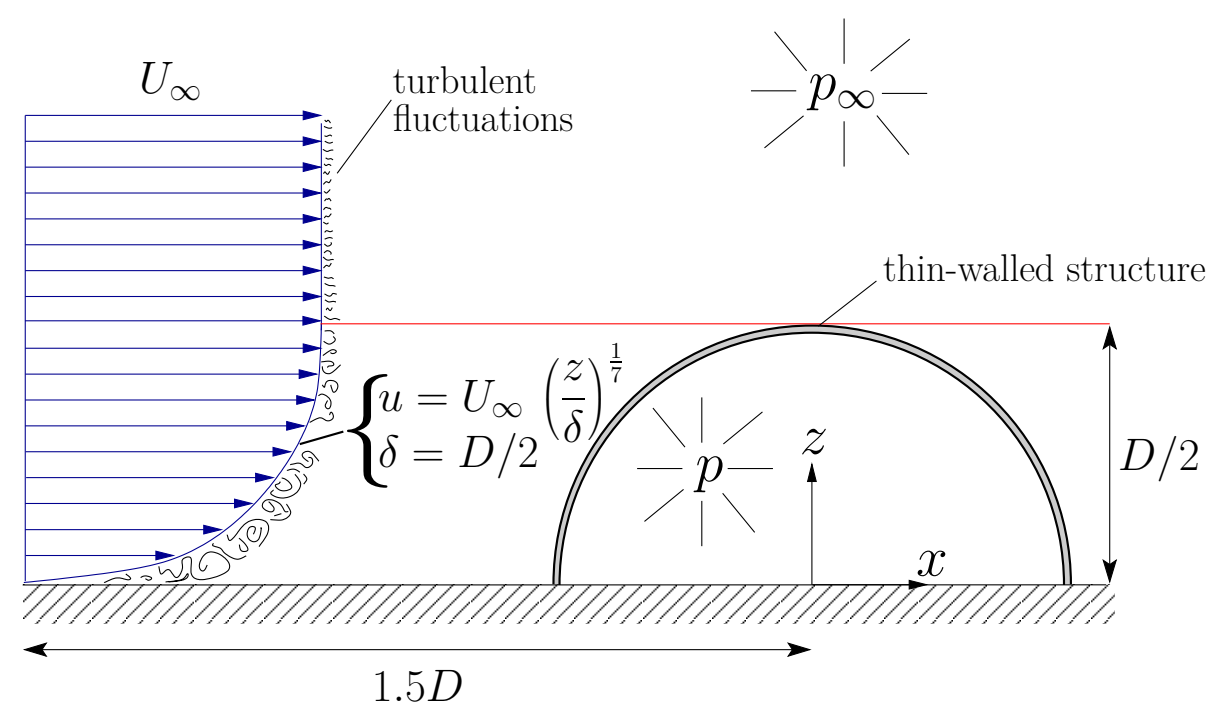

Figure 2: Pressurized flexible hemisphere within a turbulent boundary layer flow.

$U_{\infty}$ is the free-steam velocity in main flow direction outside the predefined thick turbulent boundary layer at standard conditions $\left(\rho_{\text {air }}=1.225 \mathrm{~kg} / \mathrm{m}^{3}, \mu_{\text {air }}=18.72 \times 10^{-6} \mathrm{~kg} /(\mathrm{m} \mathrm{s})\right.$ ). The wall-bounded turbulent flow follows closely a $1 / 7$ power law. The thickness of the boundary layer $\delta$ corresponds to the height of the hemisphere, i.e., $\delta=D / 2$. The origin of the Cartesian coordinate system is located at the center of the base area of the hemisphere with the corresponding flow directions: $x=$ streamwise, $y=$ spanwise and $z=$ vertical (wall-normal). In order to stabilize the thin-walled membranous structure, a pre-stress has to be applied to the membrane. Two major types of pre-stressed membranous constructions can be distinguished as categorized by Kneen (1990): The tension type and the air-supported type used here. The pre-stressing in the present case is achieved by applying the correct amount of inner pressure (Orton, 1987). The pressure inside acts normal to the membrane surface and forces an expansion of the structure. The resulting tension force in the membrane plane stiffens the structure and reduces deflections under applied compression loads.

Besides the properties of the flexible hemisphere, the current case is identical to a previous study of a rigid hemisphere presented in Wood et al. (2016) for a single Reynolds number of $\operatorname{Re}=50,000$.

\subsection{Wind tunnel and turbulent boundary layer}

The experimental investigations for the fluid-structure interaction of the flexible hemisphere are conducted in a Göttingen-type subsonic wind tunnel with an open test section of the dimensions $800 \mathrm{~mm} \times 500 \mathrm{~mm} \times 375 \mathrm{~mm}(l \times w \times h)$. The free-stream streamwise turbulence intensity is less than $0.2 \%$ over the whole velocity range of the wind tunnel $\left(u_{\max }=28 \mathrm{~m} / \mathrm{s}\right)$. The air-inflated hemisphere is immersed into a fully developed turbulent boundary layer. To achieve this, customized vortex generators are placed inside the nozzle as presented in Wood et al. (2016). 


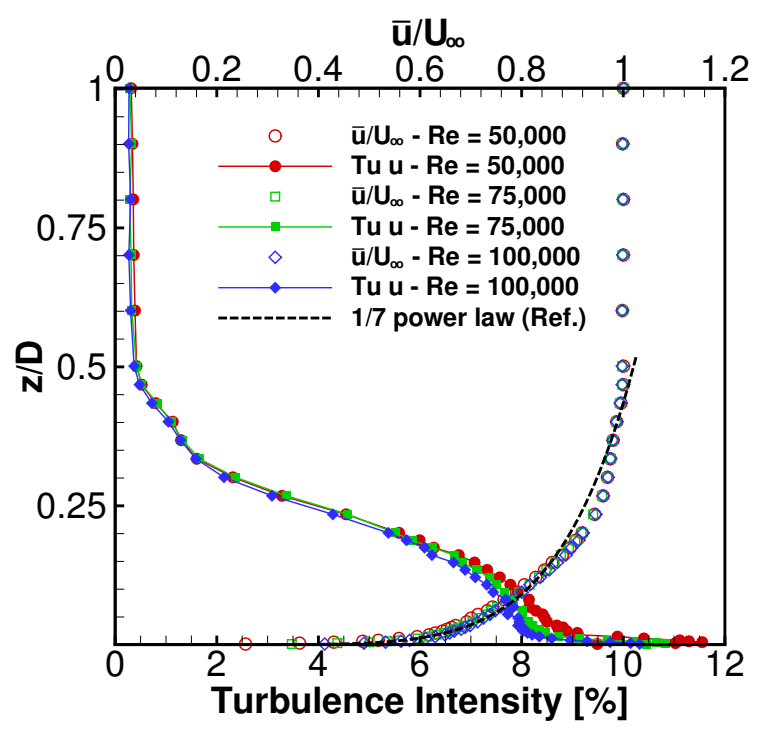

(a) Streamwise mean velocity and turbulence intensity.

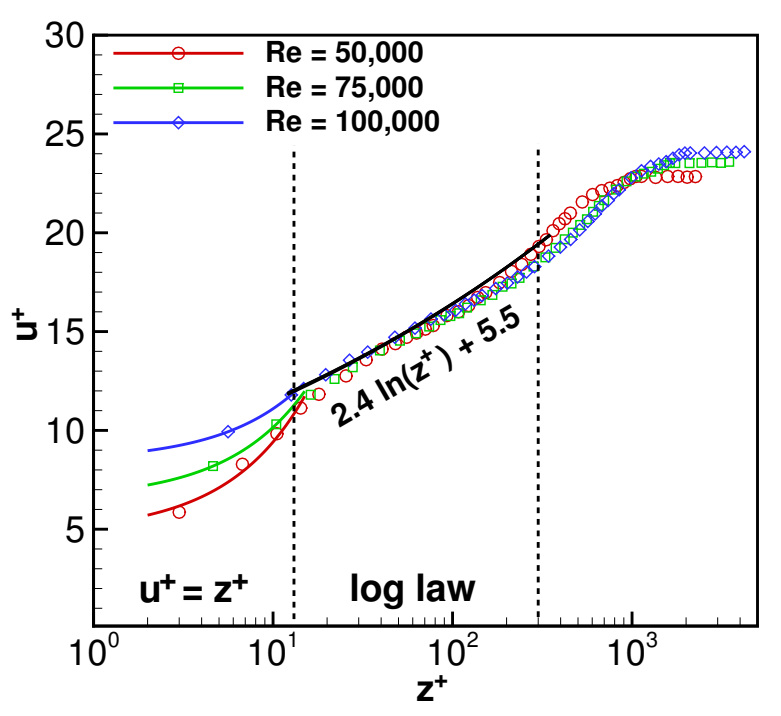

(b) $u^{+}\left(z^{+}\right)$distribution.

Figure 3: Velocity and turbulence intensity in streamwise direction measured at the inlet of the test section with a constant-temperature probe specially designed for boundary layer applications.

A comparison of the velocity and turbulence profiles of each artificially generated turbulent boundary layer is depicted in Fig. 3 .

The boundary layer characteristics are measured by constant-temperature anemometry (CTA) applying a special hot-film (platinum) probe (TSI model 1218-20) designed for this purpose. This technique offers accurate measurements of the mean velocity and the turbulent spectrum using the advantage of high sampling rates and near-wall resolution. The presented profiles are taken in the symmetry plane of the hemisphere at a location of $x / D=-1.5$ without positioning the hemisphere in the test section. The distribution of the time-averaged mean velocity profile in streamwise direction as well as the corresponding turbulence intensities are depicted in Fig. 3(a). Minor deviations occur in the turbulence profiles which are unavoidable due to the short development length from the vortex generators to the inlet of the test section $(\approx 8.2 \delta)$. Furthermore, the minor bump of the mean velocity in the region $0.25 \leq z / D \leq 0.5$ indicates a not completely developed boundary layer compared to the reference profile $(1 / 7$ power law, black dashed line). This effect was often observed in comparable experimental setups, where a sudden change of the flow is enforced by turbulence generators (Lawson, 1968; Counihan, 1969, 1975; Ligrani et al., 1979; Roberts and Walker, 2003; Sargison et al., 2004). Nevertheless, the overall profiles are similar for each Reynolds number (see Fig. 3(b)) and are thus used for the comparison of the flow field in Section 4.2. The achieved wall resolution of the CTA probe also visible in Fig. 3(b) is $z^{+}=\left(\Delta z u_{\tau}\right) / \nu=3$ corresponding to $z_{\min }=0.2 \mathrm{~mm}$ at $\operatorname{Re}=50,000$. In a previous study (Wood et al., 2016) the wall shear-stress velocity was estimated to $u_{\tau}=0.225 \mathrm{~m} / \mathrm{s}$ for $\mathrm{Re}=50,000$. This value is taken as the reference to approximate the wall shear-stress velocity $u_{\tau}$ for the higher Reynolds numbers based on the theory of a fully developed turbulent boundary layer. This is accomplished using the relation between the friction coefficient $C_{f} \sim \operatorname{Re}^{1 / 5}$ and the wall shear-stress velocity $u_{\tau}=\sqrt{C_{f} U_{\infty}^{2} / 2}$. The resulting values are given in Table 1 together with the inflow velocities and boundary layer properties at each Reynolds number $\left(\operatorname{Re}=\rho_{\text {air }} D U_{\infty} / \mu_{\text {air }}\right)$.

Furthermore, CTA is used to approximately determine the Kolmogorov length scale $\eta$ since this is a critical parameter for the setup of the PIV system. As shown by Lavoie et al. (2007) it is important to investigate the resolution of the applied PIV camera system with regard 
Journal of Fluids and Structures, vol. 80, pp. 405-440, (2018).

Table 1: Properties of the turbulent boundary layer at the examined Reynolds numbers.

\begin{tabular}{|c|c|c|c|}
\hline Reynolds number & $\mathbf{5 0 , 0 0 0}$ & $\mathbf{7 5 , 0 0 0}$ & $\mathbf{1 0 0 , 0 0 0}$ \\
\hline \hline free-stream velocity $U_{\infty}$ & $5.14 \mathrm{~m} / \mathrm{s}$ & $7.64 \mathrm{~m} / \mathrm{s}$ & $10.24 \mathrm{~m} / \mathrm{s}$ \\
\hline wall shear-stress velocity $u_{\tau}$ & $0.225 \mathrm{~m} / \mathrm{s}$ & $0.324 \mathrm{~m} / \mathrm{s}$ & $0.421 \mathrm{~m} / \mathrm{s}$ \\
\hline dimensionless wall distance $z^{+}$ & 3 & 4.3 & 5.6 \\
\hline max. turbulence level in boundary layer & $11.5 \%$ & $10.8 \%$ & $10.1 \%$ \\
\hline$\delta_{1} / \delta$ (displacement thickness) & $1 / 8$ & $1 / 10$ & $13 / 100$ \\
\hline$\delta_{2} / \delta($ momentum thickness) & $1 / 10$ & $7 / 8$ & $21 / 200$ \\
\hline$H=\delta_{1} / \delta_{2}$ (shape factor) & 1.24 & 1.27 & 1.24 \\
\hline $\operatorname{Re}_{\delta_{2}}$ (Reynolds number based on $\left.\delta_{2}\right)$ & 2530 & 3281 & 5115 \\
\hline
\end{tabular}

to its appropriate distance to the laser light sheet. Especially for a sufficient resolution of the small-scale turbulence it is necessary to adjust the size of a single control cell used by the correlation algorithm. It is recommended by Lavoie et al. (2007) to set the size of the control volume to less than $5 \eta$ for the appropriate resolution of the fine-scale turbulence. The Kolmogorov length scale is defined by $\eta=\nu^{3 / 4} / \epsilon^{1 / 4}$, where $\nu$ is the kinematic viscosity and $\epsilon$ the turbulent dissipation rate. A direct measurement of $\epsilon$ is rather difficult. In this study the approach $\epsilon=-(\bar{u} / 2)\left(\Delta \overline{\left(u^{\prime}\right)^{2}} / \Delta z\right)$ proposed by Lavoie et al. (2007) is used for a rough approximation of $\eta$. Here $\bar{u}$ is the mean velocity and $\overline{\left(u^{\prime}\right)^{2}}$ a measure for the turbulent kinetic energy. This simplification is only valid for a local isotropic turbulence distribution and leads to a Kolmogorov length scale of $\eta \approx 0.3 \mathrm{~mm}$ for the present study.

\subsection{Model of the flexible hemisphere}

Two major aspects are considered while manufacturing the model: First, the thickness of

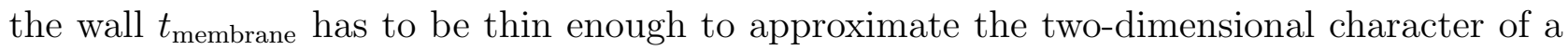
membrane and to allow perceptible structural deformations under the considered wind load. Second, the size (diameter $D$ ) and the surface roughness $R_{a}$ should be comparable to the rigid model in order to allow a direct comparison with the rigid case. To satisfy both requirements to the same extent is challenging, since most suitable thin structures, such as plastic films, tend to wrinkle when brought into a specific three-dimensional shape affecting the form and roughness. Especially smooth curved surfaces are difficult to produce. Furthermore, the dimension of the wind tunnel model is relatively small $(D=150 \mathrm{~mm})$. This directly affects the average thickness $\bar{t}_{\text {membrane }}=0.16 \mathrm{~mm}$, which has to be scaled accordingly.

To tackle these issues, the casting process depicted in Fig. 4(a) is chosen to manufacture an adequate model. The corresponding casting mold is made of two main elements: a positive and a negative hemispherical form. The contour of the negative shape is adjusted to the dimensions of the rigid hemisphere, i.e., $D=D_{\text {neg }}=150 \mathrm{~mm}$, to ensure geometrical identity between the flexible and the rigid model. The positive form is set to a diameter of $D_{\text {pos }}=149.6 \mathrm{~mm}$ so that a gap of about $0.2 \mathrm{~mm}$ between both shapes arises, which corresponds to the thickness $t_{\text {membrane }}$ of the flexible model. The minimum thickness of the wall is limited by the fabrication tolerances of the used milling machine.

During the casting process liquid silicone Elastosil@ E625 A/B RT ${ }^{4}$ in connection with a hardener in a mixture ratio of 9:1 is poured into the negative shape (see Fig. 4(a)). Afterwards, the positive and the negative forms are pressed together in order to force the silicone to flow freely within the gap. Three outlets at the upper side of the casting mold enable surplus

${ }^{4}$ http://www. wacker.com 


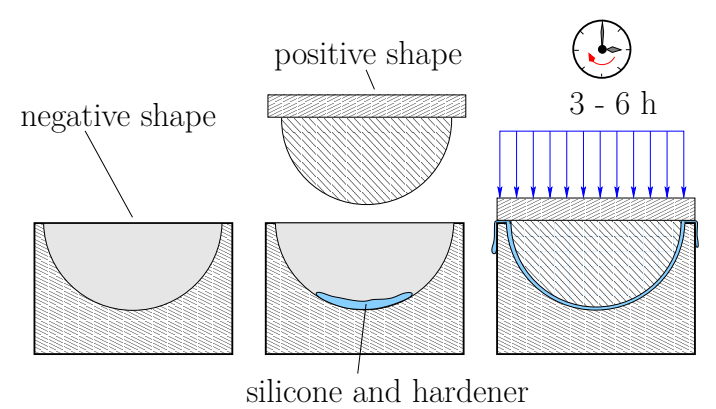

(a) Casting process.

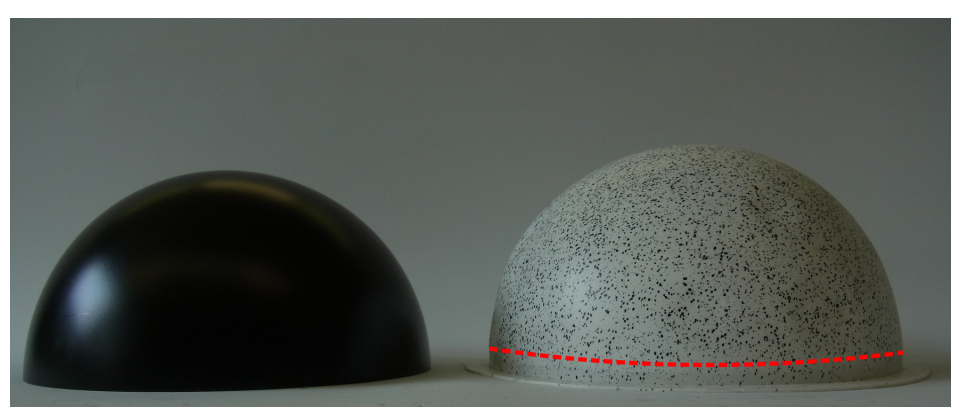

(b) Rigid (left) and flexible (right) model of the hemisphere.

Figure 4: Production of the flexible hemisphere model.

silicone to flow out. The curing process lasts between 6 and 48 hours. Longer curing times were accomplished by an add-on inhibitor delaying the hardening process. The inhibitor was used in order to reduce the thickness of the membrane as the silicone was able to flow longer out of the casting form. A comparison between the rigid and the flexible model is depicted in Fig. 4(b). The overall size of the flexible model is identical to the rigid hemisphere. Additionally, the flexible model has a cylindrical extension with a pedestal foot for the connection to the flat plate which is placed into the test section of the wind tunnel. The separation between the hemispherical shape and the additional part is indicated by the red dashed line. The latter is enwrapped into the flat plate. Thus, during the experiments only the hemispherical part of the model is of relevance. To determine the mass of the hemispherical part of the flexible model, the support (cylindrical extension and the pedestal foot) has to be subtracted from the total mass. For this purpose, a model comparable in mass and thickness to the one applied in the present study, was cut apart. The mass of the hemispherical part was measured to be $m_{\text {membrane }}^{\exp }=6.22 \times 10^{-3} \mathrm{~kg}$. To validate this result, the analytically predicted volume of a hollow hemisphere based on the average thickness of the wall $\bar{t}_{\text {membrane }}$ is multiplied by the density of the silicone leading to a theoretical mass of $m_{\text {membrane }}^{\text {theory }}=6.11 \times 10^{-3} \mathrm{~kg}$. 26 models of the flexible hemisphere were produced showing the reproducibility of the manufacturing procedure. For the sake of brevity the production process and the corresponding evaluation can be found in Appendix A including the static and dynamic behavior of the flexible model at various gauge pressures.

Finally, the material properties of the applied silicone are determined by utilizing a standard

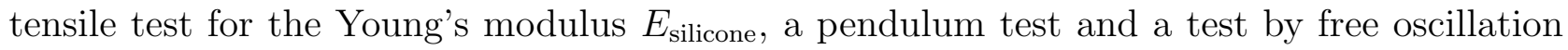
of the air-inflated system in the wind tunnel setup to determine the damping ratio $b$. The characteristic Young's modulus is evaluated by linearizing the stress-strain correlation of the tensile test (see Fig. B.30(a)) in the specific strain region $0 \leq \varepsilon \leq 0.1$ to $E_{\text {silicone }} \approx 7 \times 10^{5} \mathrm{~Pa}$. Strain measurements during the wind tunnel tests were well below this value. Furthermore, a Poisson ratio of 0.45 was determined in good agreement with the literature. An average system damping ratio of $\bar{b}_{\text {system }} \approx 0.054$ was measured based on the results of the pre-stressed hemisphere. Detailed measurements of these material properties are listed in Appendix B. The specifications and settings of the final model of the flexible hemisphere used during this study are given in Table 2 .

\subsection{Comparison with the rigid hemisphere}

The flow around the rigid hemisphere is used to identify differences in the averaged flow field between the solid and the flexible structure. It is assumed that the flow-induced displacements of the flexible hemisphere have a significant impact on the flow characteristics. 
Journal of Fluids and Structures, vol. 80, pp. 405-440, (2018).

Table 2: Specifications of the applied flexible hemisphere model.

\begin{tabular}{|c|c|}
\hline feature & dimension \\
\hline \hline diameter & $D=150 \mathrm{~mm} \pm 0.04 \mathrm{~mm}$ \\
\hline average wall thickness & $\bar{t}_{\text {membrane }}=0.16 \mathrm{~mm}$ \\
\hline mass of hemispherical part & $m_{\text {membrane }} \approx 6.2 \times 10^{-3} \mathrm{~kg}$ \\
\hline density & $\rho_{\text {silicone }}=1050 \mathrm{~kg} / \mathrm{m}^{3}$ \\
\hline characteristic Young's modulus & $E_{\text {silicone }} \approx 7 \times 10^{5} \mathrm{~Pa}$ \\
\hline Poisson ratio & 0.45 \\
\hline damping ratio & $\bar{b}_{\text {system }} \approx 0.054$ \\
\hline & $\Delta p_{\mathrm{FSI}_{1}}=19 \mathrm{~Pa}(\mathrm{Re}=50,000)$ \\
inner gauge pressure & $\Delta p_{\mathrm{FSI}_{2}}=34 \mathrm{~Pa}(\mathrm{Re}=75,000)$ \\
(see Appendix A.3) & $\Delta p_{\mathrm{FSI}_{3}}=43 \mathrm{~Pa}(\mathrm{Re}=100,000)$ \\
\hline
\end{tabular}

The rigid hemisphere has already been investigated at $R e=50,000$ in Wood et al. (2016) using laser-Doppler anemometry (LDA). Nevertheless, to ensure an accurate comparison between the flexible and the rigid structure, it is necessary to repeat the measurements of the rigid case within a matching framework. For this purpose, the flow field of the rigid hemisphere is measured once again with the equipment presented in the next section. It is meaningful to compare both configurations with the same spatial resolution. This procedure minimizes interpolation errors that arise when comparing measurement results that base on different spatial resolutions. In a preliminary study the former LDA results were used to validate the suitability of the applied particle-image-velocimetry. Both measurement systems showed nearly identical results.

\section{Measurement techniques for fluid-structure interaction}

\subsection{Flow field measurements}

\subsubsection{Particle-Image-Velocimetry}

Particle-Image-Velocimetry (PIV) is used for the acquisition of the global time-averaged flow field and Reynolds stresses. The setup of the PIV measurements for the flexible model is schematically shown in Fig. 5(a). The same setup is applied for the rigid case. The flow around the surface-mounted model is evaluated in the symmetry plane by applying a high spatial resolution mono-PIV equipment consisting of a CCD camera (TSI PowerView ${ }^{\mathrm{TM}}$ 29MP), a pulse laser (Litron NanoPIV L, 200mJ, 15Hz) and a synchronizer (TSI Laserpulse Synchronizer). The components are connected to a workstation with TSI Insight $4 \mathrm{G}^{5}$ software. The laser light sheet is guided to a mirror adjusted to the symmetry plane of the model. In this setup the $u$ - (streamwise) and $w$-velocity component (wall-normal) of the flow can be measured. The measurement plane is divided into two overlapping areas of interest. In the first area the CCD camera focuses on the inflow and the close vicinity of the hemisphere. It captures the area $-1.5 \leq x / D \leq 0.7$ in streamwise and $0 \leq z / D \leq 1$ in wall-normal direction. The second area records the wake regime at $0 \leq x / D \leq 2$ and $0 \leq z / D \leq 1$. For an optimum fitting with minimum misalignment errors between both evaluation planes the images are brought together at $x / D=0$. To ensure comparable results between each measurement, the camera position is altered by a high-precision traversing system with programmable coordinates for optimum

\footnotetext{
${ }^{5}$ http://www.tsi.com/insight4g.aspx
} 


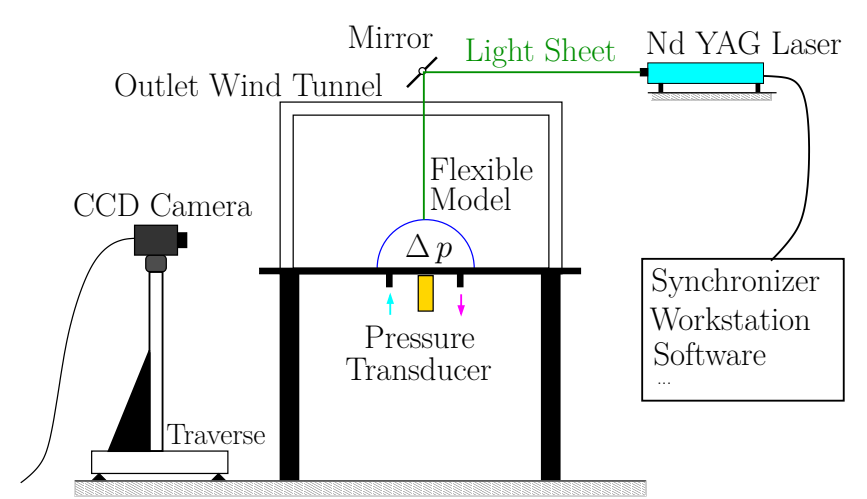

(a) Setup for PIV (fluid measurements).

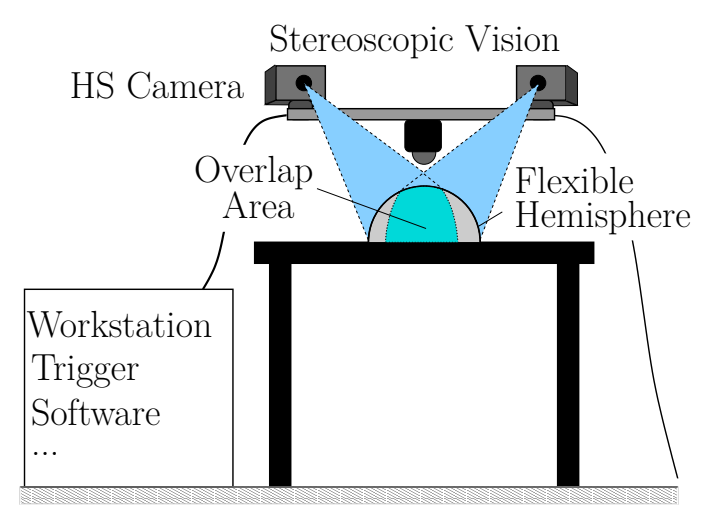

(b) Setup for DIC (structure measurements).

Figure 5: Schematic representation of the utilized measurement setups for the fluid-structure interaction.

reproducibility. The applied camera settings allow a maximum frame rate of $1.67 \mathrm{fps}$ (straddle mode) at the highest resolution $(6400 \times 4400$ pixels; 12 bits per pixel). The time interval between each image pair is set to $\Delta t_{\mathrm{PIV}}=50 \mu \mathrm{s}$. Following the recommendations of Lavoie et al. (2007) the spot dimensions are set to $24 \times 24$ pixels. With a calibration factor of $63.26 \mu \mathrm{m} /$ pixel this leads to an overall cell size of $1.518 \mathrm{~mm} \times 1.518 \mathrm{~mm}$. With regard to the approximated Kolmogorov length scale this translates to about $5 \eta \times 5 \eta$. The evaluation of the fluid field is performed with a grid size of 39,066 evaluation cells. Furthermore, a $50 \%$ overlap on a standard Nyquist grid is applied using a FFT-correlation algorithm with a bi-linear peak function. To increase the image quality, a gray-scale filter is used as pre-processor. Finally, a post-processing step is applied to reduce the effect of bad vector calculation by neighborhood replacement and local smoothing functions. Each measurement contains 1500 double-images captured during a measurement time of about 15 minutes. This leads to an adequate time-average for the evaluation of the first- and second-order moments. Di-Ethyl-Hexyl-Sebacat (DEHS) droplets are used as seeding medium generated by a TSI six-jet atomizer at the receiver of the wind tunnel. The average droplet size is estimated to about $0.5 \mu \mathrm{m}$. Background reflections of the laser light at the outlet walls of the nozzle were observed in the present wind tunnel. Hence, in the region at about $x / D=-1.4$ no meaningful vectors could be detected by the correlation algorithm. These errors are mostly visible as a discontinuity in the results of the streamwise velocity $\bar{u} / U_{\infty}$ of the flow field.

\subsubsection{Constant-temperature anemometry}

A constant-temperature platinum film probe (TSI model 1218) is used to measure the velocity spectra at specific monitoring points in the wake near the rigid and moving surface of each hemisphere model (see Section 3.2.2). It is assumed that the oscillations of the flexible wall have an impact on the velocity field close to the surface. Additionally, the velocity spectra are brought into correlation with the structure oscillations measured by the high-speed cameras in order to analyze and identify possible sources of FSI phenomena. Measurements are performed at a sampling rate of $2000 \mathrm{~Hz}$. An interval of 2 seconds is used to collect a data set containing 4000 single measurements to evaluate the power spectral density of the velocity signal. This procedure is repeated 1000 times to receive a time-independent average of the frequency spectrum at each monitoring point. 
Journal of Fluids and Structures, vol. 80, pp. 405-440, (2018).

\subsection{Structure deformation measurements}

\subsubsection{Digital-Image Correlation}

The structure deformations are measured by the Digital-Image Correlation (DIC) technique based on a pair of high-speed cameras (SpeedCam MacroVis EoSens) schematically depicted in Fig. 5(b). Due to the stereoscopic mounting each camera takes the flexible hemisphere under a certain angle into focus. The chosen angle defines the overlapping between both camera images which is directly connected to the correlation region. The flexible model requires a speckle pattern (see Fig. 4(b)) which has to be sprayed onto the membrane. This is necessary for the applied correlation algorithm which compares the dot pattern of a chosen reference image (undeformed hemisphere) with an arbitrary image of the deformed structure. The measurements are synchronized and controlled by the software VisArt ${ }^{6}$. The actual evaluation of the deformations of each image-pair is done by the software tool Istra $4 \mathrm{D}^{7}$. The measurements of the oscillating structure are performed at $250 \mathrm{fps}$ with an image resolution of $1503 \times 996$ pixels. This assures spatially highly resolved images at a maximum frequency resolution of $125 \mathrm{~Hz}$ according to the Nyquist criterion (Nyquist, 2002; Grenander et al., 1959; Mishali and Eldar, 2010). This frame rate is chosen based on the following motivation: A preliminary dynamic response test of the pressurized flexible hemisphere conducted outside the wind tunnel revealed significant natural frequencies in the range $10 \mathrm{~Hz} \leq f \leq 120 \mathrm{~Hz}$ (see Section 4.1). It is assumed that the unsteady response of the flexible membrane to wind loads and hereto connected FSI phenomena, such as vortex-induced vibrations, occur within this frequency range. Furthermore, a certain amount of time-independent data is needed to generate a meaningful statistical estimation of the involved frequencies of the structural movement. Based on the applied image resolution and frame rate the available memory allows a data sample of 5611 double images covering a time span of 22.44 seconds.

\subsubsection{Monitoring of the structure deformation}

The present study primarily concentrates on the deformations appearing on the lee-side of the flexible hemisphere. A map of the chosen monitoring points on the membrane surface is depicted in Fig. 6. Each point is defined by an angle $\varphi$ referring to the base area. The points are located at increments of $\Delta \varphi=15^{\circ}$ from the bottom to the apex in the symmetry plane of the hemisphere. Additionally, they are denoted by the compass notation. As the flow passes the test section from left to right (west to east) the wake region is connected to the east-side. In the following each monitoring point is identified as E15, E30, E45, E60, E75 and apex. In case of the constant temperature anemometry the velocity spectra close to the wall are recorded at the same points for the rigid and flexible hemisphere at a distance of about $2 \mathrm{~mm}$ from the surface.

\subsection{Additional instrumentation and considerations for FSI}

\subsubsection{Pressure probe implementation}

To measure the pressure inside the flexible hemisphere, the dynamic pressure transducer WIKA Type SL-1 is utilized. Its measuring range is between $0 \leq \Delta p \leq 2500 \mathrm{~Pa}$. It was calibrated according to the DAkkS $\mathrm{S}^{8}$ guide line. A Betz manometer was used as calibration standard. The maximum relative measurement uncertainty in the chosen pressure range of this study $(0 \leq \Delta p \leq 300 \mathrm{~Pa})$ is $2.3 \%$.

\footnotetext{
${ }^{6}$ http://www.hsvision.de/en/products/software/visart-detail

${ }^{7}$ https://www . dantecdynamics.com/istra-4d-shearography

${ }^{8}$ http://www.dakks.de/sites/default/files/dakks-dkd-r_6-1_20100614_v1.0.pdf
} 


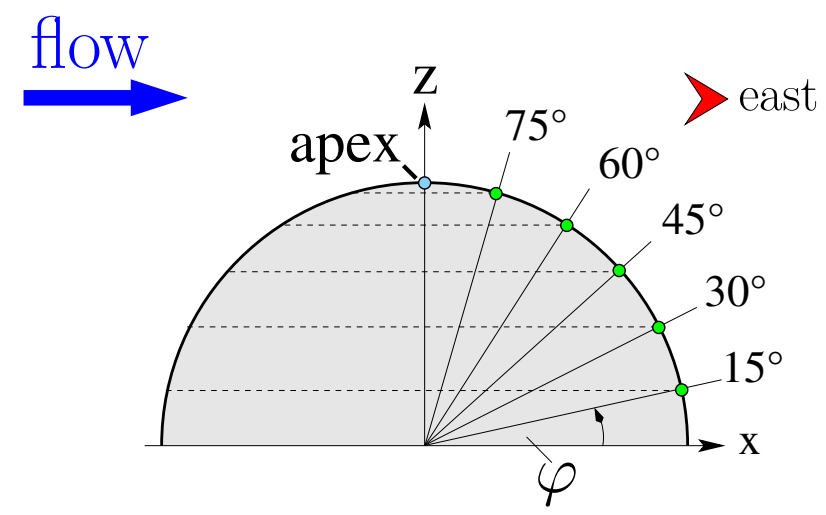

Figure 6: Mapping of the monitoring points on the surface in the wake region (east-side) and at the apex of the flexible hemisphere.

\subsubsection{Non-synchronized FSI measurements}

The flow field and the structure deformation measurements are conducted separately. The reason for this is based on the different requirements for each measurement device. The high-speed measurements of the structure deformations require a powerful light source to ensure adequate illumination for the short shutter times of the camera system and to avoid dark images that cannot be used for the correlation software. On the other hand, the PIV measurement depends on a high intensity laser light sheet to enlighten the small DEHS droplets in the flow. In this case the environment should be as dark as possible to ensure that the CCD camera receives enough scattered laser light of the tracers.

\section{Experimental results and discussion}

\subsection{Dynamic response characteristics of the flexible hemisphere}

Before the actual measurements of the flexible hemisphere are conducted, the structural behavior of the silicone model is analyzed. A setup capable to determine the structural response of the hemisphere outside the wind tunnel is of interest to characterize the natural frequencies without other excitation sources such as wind loads. For this purpose, a standardized dynamic response test is applied.

The experimental setup is depicted in Fig. 7. The model of the flexible hemisphere is inflated by the calibration pressure of $\Delta p_{\text {cal }}=p-p_{\infty}=43 \mathrm{~Pa}$ to ensure a best fit to a perfect hemispherical shape. An impulse is applied to the structure of the hemisphere by means of a triggering device schematically presented in Fig. 7(a). The device consists of a small rod (pointer) and a spring which are encased in a cylindrical housing. The rod can be pushed extending the spring until it reaches a point where it clinches into position and protrudes maximally out of the housing. A button at the side of the housing is installed to release the rod. When activating the button, the rod is pulled rapidly backwards into the housing by the elastic force of the spring and the flexible structure can move freely. This procedure is considered to be close to a free oscillation of the body with no external influences other than the impulse initiated by the trigger.

The trigger device is guided by an arc with predefined drillings (see Fig. 7(b)) that point radially to the center of the base area of the hemisphere to assure normal contact between the pointer and the flexible surface. The tip of the pointer has a diameter of $1 \mathrm{~mm}$. The impulse is triggered at a surface point located at an angle of $45^{\circ}$. The excitation of the structure is monitored at the apex of the hemisphere. The test procedure is as follows: The pre-stressed pointer is pressed onto the surface of the hemisphere with a pre-defined deformation between 5 and $7 \mathrm{~mm}$. 


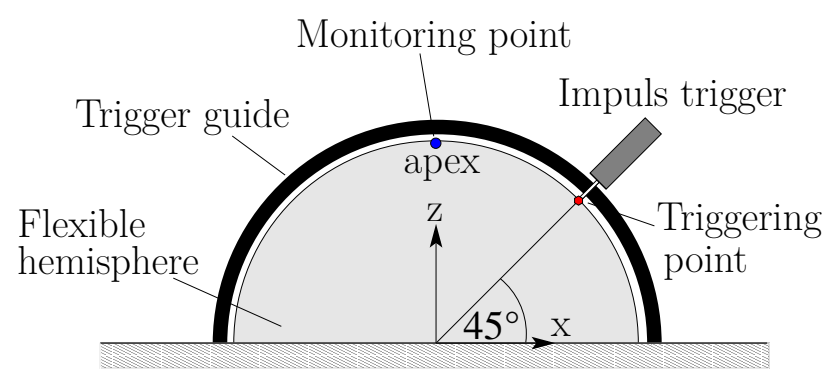

(a) Schematic view of the setup.

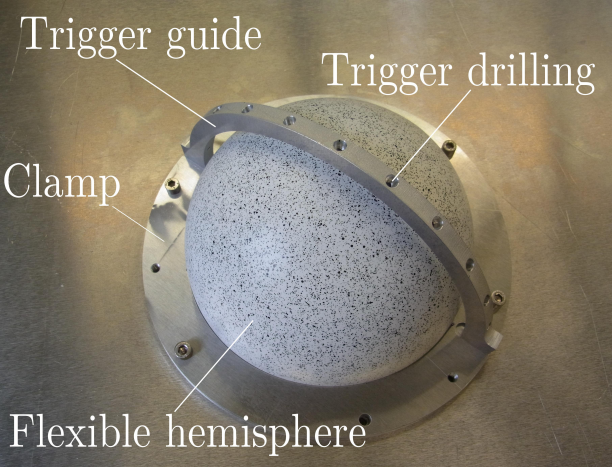

(b) Actual trigger guide system.
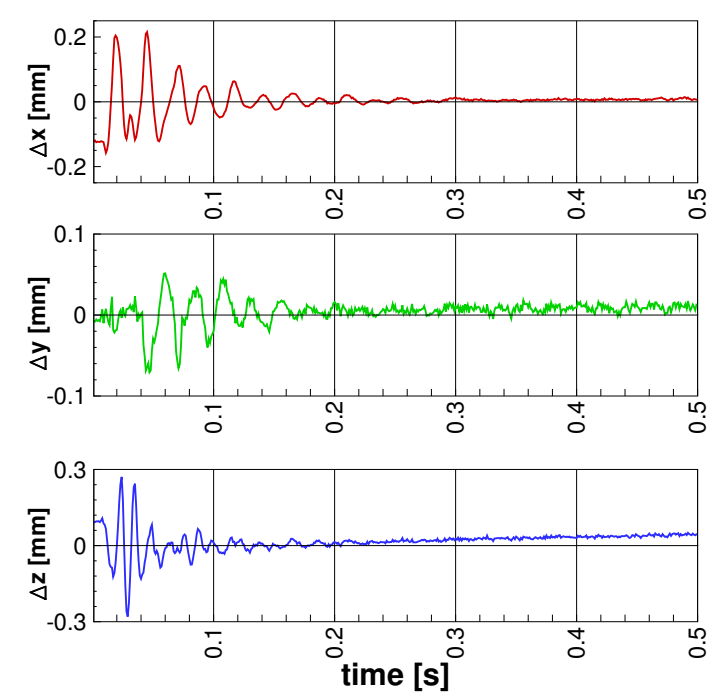

(c) Dynamic response at the apex.

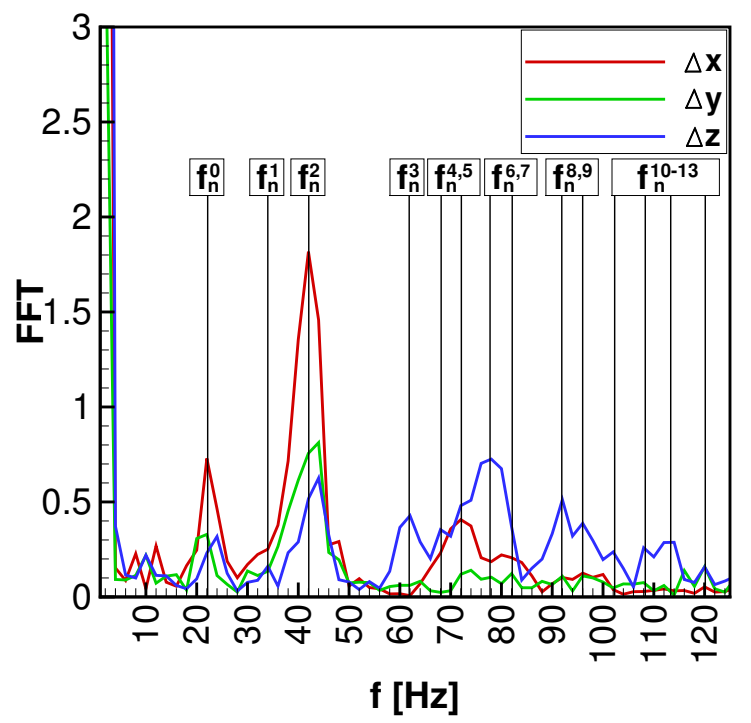

(d) Frequency spectra of eigenfrequencies.

Figure 7: Setup used for the standardized dynamic response test with a fixed impulse trigger at $45^{\circ}$ and the monitoring point at the apex of the flexible hemisphere.

After exciting the structure high-speed digital-image correlation measurements are performed at $1000 \mathrm{fps}$ to capture the $\Delta x-, \Delta y$ - and $\Delta z$-components of the structure displacements as a function of time as depicted in Fig. 7(c). Based on these data the dynamic response of the structure is characterized by applying a fast Fourier transformation leading to the natural frequencies of the flexible hemisphere presented in Fig. 7(d). The dominant natural frequencies of the flexible hemisphere are listed in Table 3.

Table 3: Frequency response of the flexible hemisphere from the trigger test in the range $10 \mathrm{~Hz} \leq f_{n} \leq 120 \mathrm{~Hz}$.

\begin{tabular}{|c|c|c|c|c|c|c|c|c|}
\hline Eigenfrequencies & $f_{n}^{0}$ & $f_{n}^{1}$ & $f_{n}^{2}$ & $f_{n}^{3}$ & $f_{n}^{4,5}$ & $f_{n}^{6,7}$ & $f_{n}^{8,9}$ & $f_{n}^{10-13}$ \\
\hline Value $[\mathrm{Hz}]$ & 22 & 35 & 42 & 62 & 68,72 & 78,82 & 92,96 & $102,108,113,120$ \\
\hline
\end{tabular}

The decomposition of the monitoring point into three components $(\Delta x, \Delta y$ and $\Delta z)$ reveals an improved resolution of the spectra compared to the total displacements $\Delta_{\text {total }}$ that tends to blur single frequencies to broader peaks. Furthermore, a strong oscillation in one direction will also cause amplitudes of the other components since the movement of the monitoring point is a coupled three-dimensional effect, for example visible at $f_{n}^{2}=42 \mathrm{~Hz}$.

Note that the very low frequency at about $1.5 \mathrm{~Hz}$ is not a natural frequency. It is a result of the re-inflation of the hemisphere. This process can be best seen in the $z$-component in Fig. 7(c). Directly after the trigger rod is released from the flexible structure, an equalization of the inner 
pressure sets in. After a while the membrane reaches a steady-state again. This process takes more time than the natural frequency response but is still captured by the high-speed cameras during the measurement.

\subsection{Time-averaged flow field characteristics}

In the following the time-averaged flow around the rigid and the flexible hemisphere are compared. The streamwise and wall-normal velocity components as well as the Reynolds stresses are evaluated to find differences between both configurations.

Figure 8 depicts the results of the PIV measurement at $R e=50,000$. Note that a detailed description of the characteristic features of the flow field can be found in Wood et al. (2016). In this case marginal differences in the flow field between the rigid and the flexible hemisphere are recognizable. These are visible in the second-order moments $\overline{w^{\prime} w^{\prime}} / U_{\infty}^{2}$ (Figs. $8(\mathrm{~g})$ and $8(\mathrm{~h})$ ) and $\overline{u^{\prime} w^{\prime}} / U_{\infty}^{2}$ (Figs. 8(i) and 8(j)). The Reynolds stresses close to the surface of the body at the wake side of the flexible hemisphere are slightly lower. At this moderate flow velocity $\left(U_{\infty}=5.14 \mathrm{~m} / \mathrm{s}\right)$ only very small deformations of the flexible model occur. These are in the order of magnitude of $10^{-4} \mathrm{~m}$ and have only a weak impact on the flow field. Besides these deviations, the result ensures a good geometric matching between the rigid and the flexible model and underlines the negligibility of the variations of the surface roughness for the present case (see Appendix A.2).

The deviations of the flow characteristics between the solid and the deformable structure increase visibly at the next higher free-stream velocity of $U_{\infty}=7.64 \mathrm{~m} / \mathrm{s}(\mathrm{Re}=75,000)$ presented in Fig. 9. In this case differences in the time-averaged velocity components as well as the Reynolds stresses are observed, especially in the wake region. The recirculation area behind the rigid hemisphere in Fig. 9(a) is more compact compared with the flexible model in Fig. 9(b) which has a pronounced "belly shape", similar to the former results at Re $=50,000$. This can be explained by the nearly fixed detachment line on the rigid model leading to the development of a pronounced vertical velocity distribution depicted in Fig. 9(c). In contrast, the deformable surface of the flexible hemisphere adapts to the unsteady flow conditions. The vertical flow past the deformable structure is less distinctive compared to the rigid case. The Reynolds stresses in the wake of the flexible hemisphere are slightly attenuated compared to the rigid case. The maximum intensities of the streamwise Reynolds stresses $\overline{u^{\prime} u^{\prime}} / U_{\infty}^{2}$ (Figs. 9(e) and 9(f)) form a narrower region at $x / D=0.75$ compared to the rigid case. The wall-normal component $\overline{w^{\prime} w^{\prime}} / U_{\infty}^{2}$ (Figs. $9(\mathrm{~g})$ and $9(\mathrm{~h})$ ) is not as distinctive in the free shear layer of the deformable model yielding a slender distribution. Special attention should be paid to the near-wall behavior of the shear stress component $\overline{u^{\prime} w^{\prime}} / U_{\infty}^{2}$ (Figs. 9(i) and 9(j)). While the rigid hemisphere shows a nearly constant distribution along the complete measured distance in the near-ground region, the fluctuations of the flexible case have a locally restricted maximum between $0.75 \leq x / D \leq$ 1.15. The source of this effect is not clearly traceable based on the two-dimensional PIV data. The results at $R e=100,000$ are presented in Fig. 10. All components follow qualitatively the trend mentioned for $\mathrm{Re}=75,000$. The recirculation area is forming closer to the rear side of the rigid model compared to the flexible hemisphere. The significantly different distribution of the wall-normal velocity component $\bar{w} / U_{\infty}$ (Figs. 10(c) and 10(d)) of the flexible model is a result of the strong global streamwise and lateral movement of the deformable surface. The rigid body guides the oncoming fluid along its solid surface and with increasing Reynolds number the detachment point shifts to the back of the hemisphere leading to a smaller recirculation area. This is not the case for the flexible hemisphere. The detachment of the flow from the surface is therefore depending on the adapted shape of the flexible body, which is no longer comparable to a perfect hemisphere. The shape of the deformed body can be considered as 


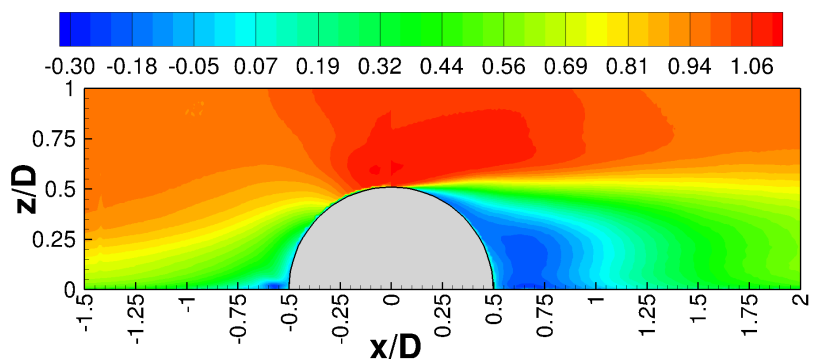

(a) $\bar{u} / U_{\infty}$ rigid

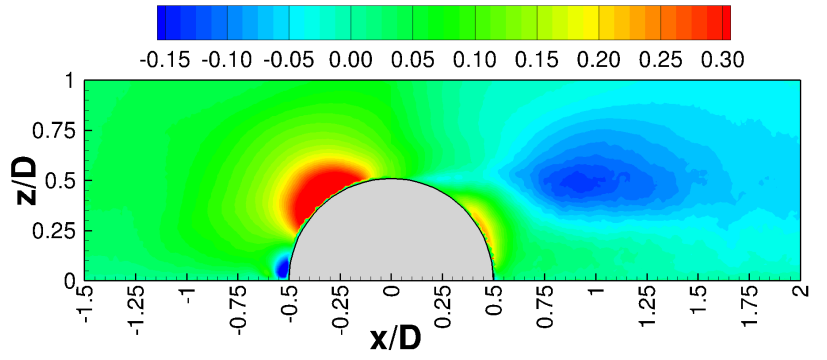

(c) $\bar{w} / U_{\infty}$ rigid

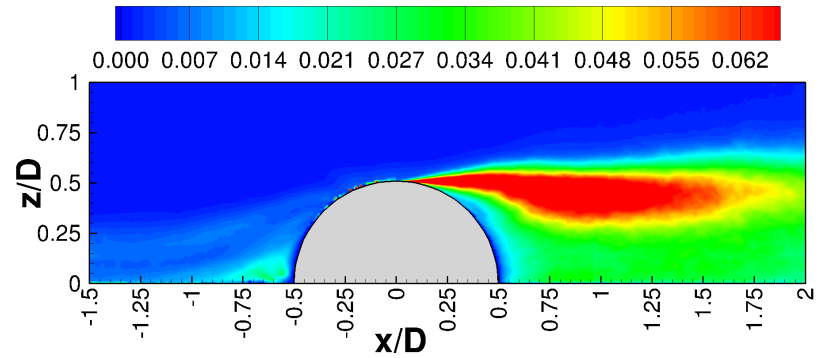

(e) $\overline{u^{\prime} u^{\prime}} / U_{\infty}^{2}$ rigid

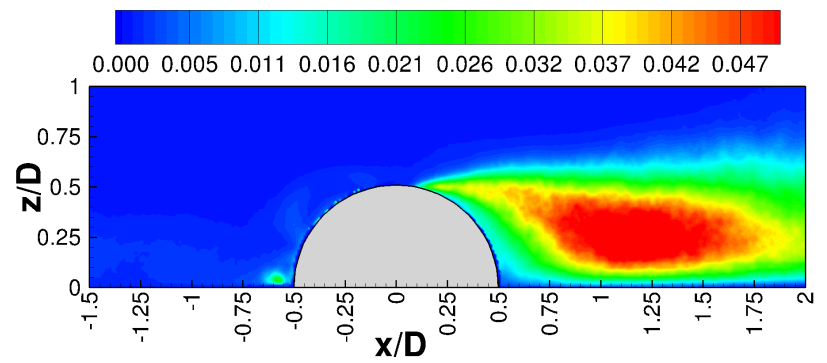

(g) $\overline{w^{\prime} w^{\prime}} / U_{\infty}^{2}$ rigid

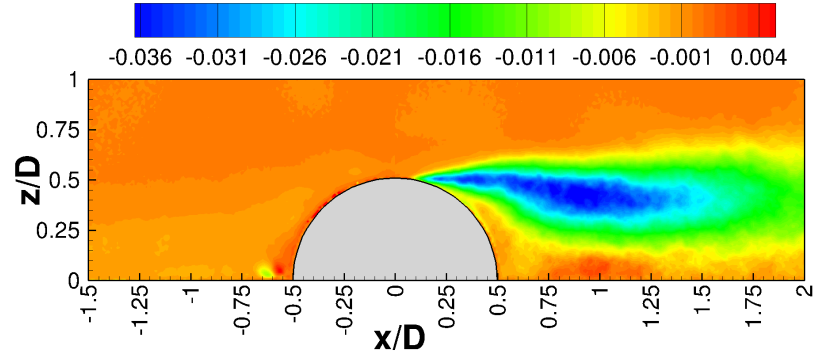

(i) $\overline{u^{\prime} w^{\prime}} / U_{\infty}^{2}$ rigid

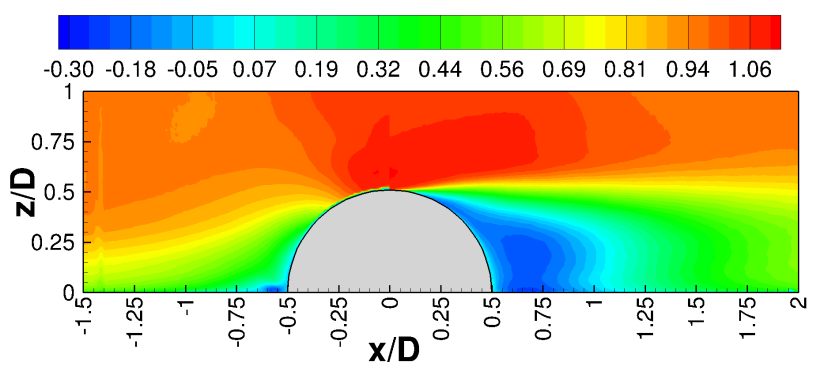

(b) $\bar{u} / U_{\infty}$ flexible

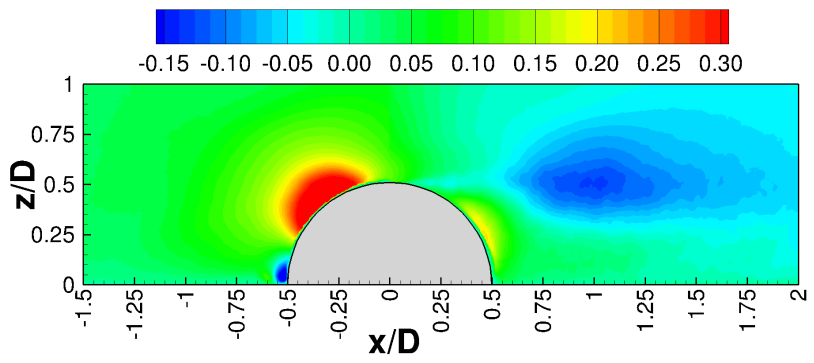

(d) $\bar{w} / U_{\infty}$ flexible

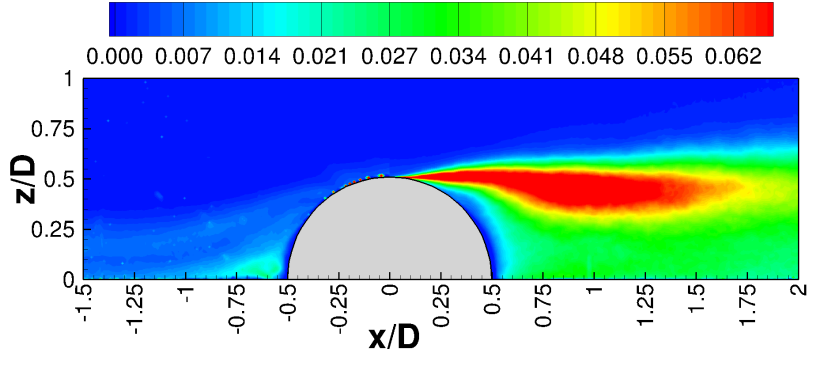

(f) $\overline{u^{\prime} u^{\prime}} / U_{\infty}^{2}$ flexible

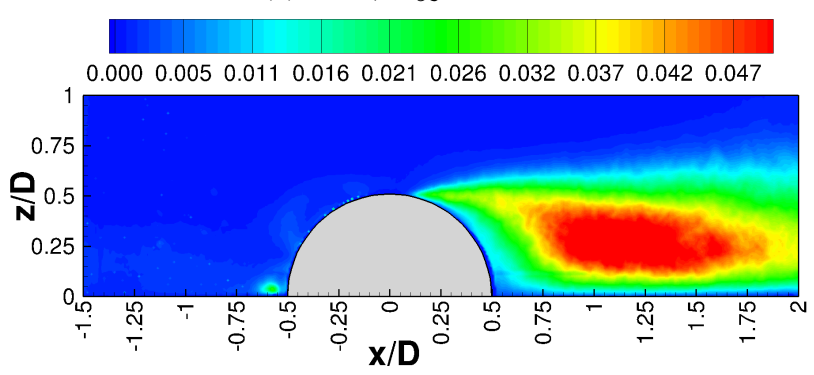

(h) $\overline{w^{\prime} w^{\prime}} / U_{\infty}^{2}$ flexible

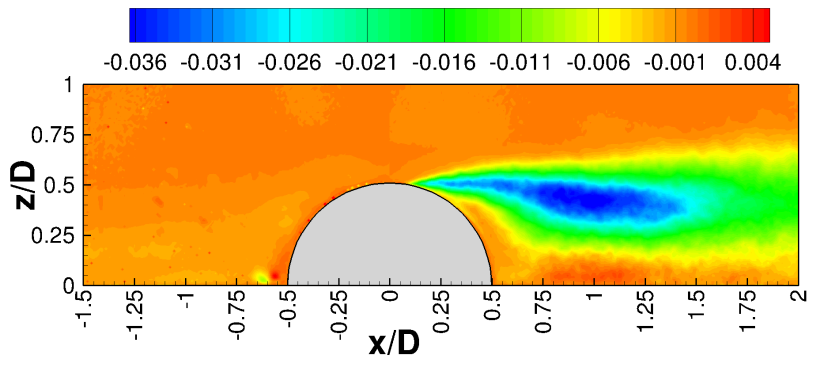

(j) $\overline{u^{\prime} w^{\prime}} / U_{\infty}^{2}$ flexible

Figure 8: Comparison of the time-averaged flow field characteristics of the rigid and the flexible hemisphere in the symmetry $x-z-$ plane at $y / D=0$ at $\operatorname{Re}=50,000$. 


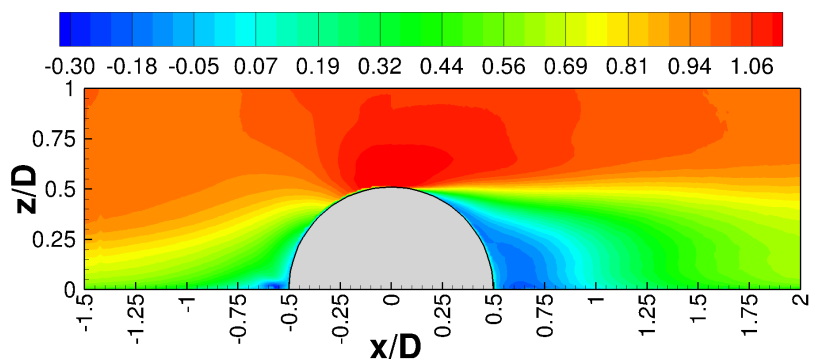

(a) $\bar{u} / U_{\infty}$ rigid

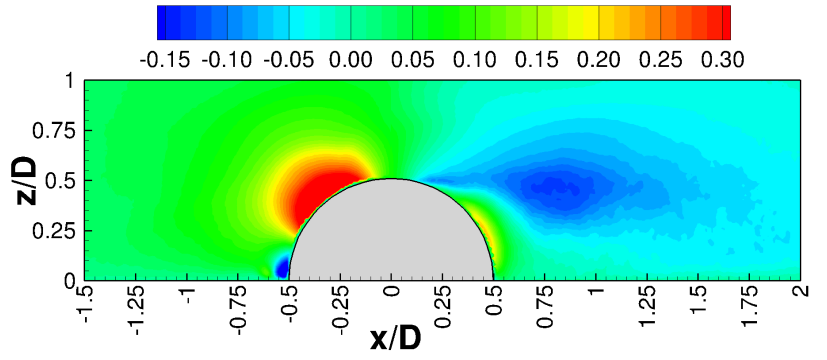

(c) $\bar{w} / U_{\infty}$ rigid

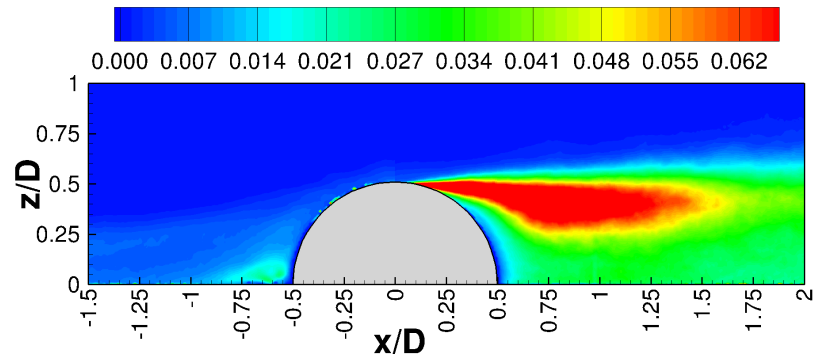

(e) $\overline{u^{\prime} u^{\prime}} / U_{\infty}^{2}$ rigid

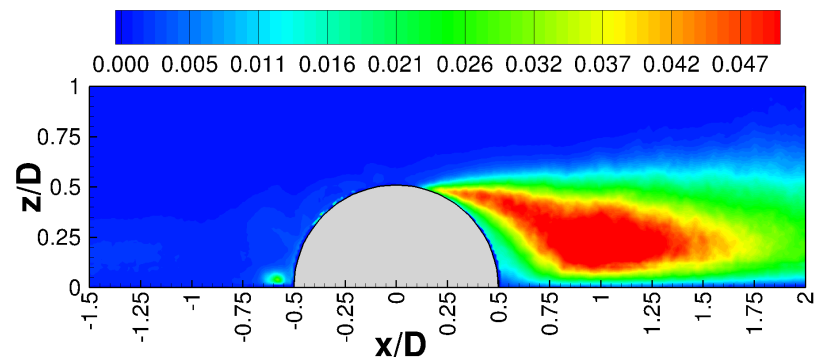

(g) $\overline{w^{\prime} w^{\prime}} / U_{\infty}^{2}$ rigid

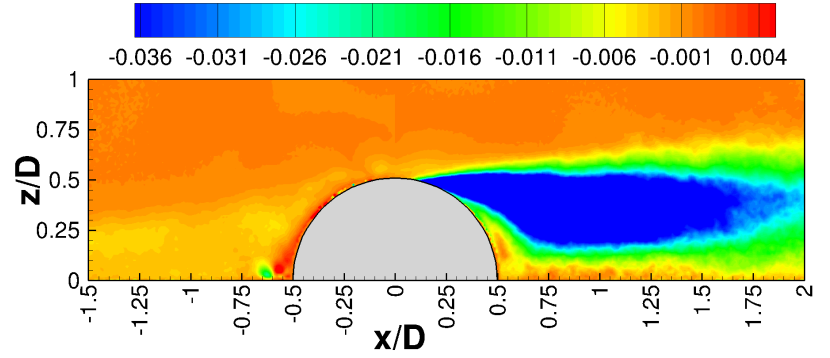

(i) $\overline{u^{\prime} w^{\prime}} / U_{\infty}^{2}$ rigid

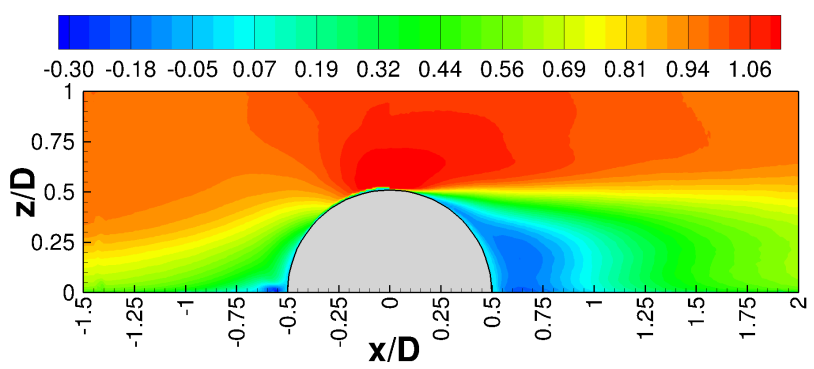

(b) $\bar{u} / U_{\infty}$ flexible

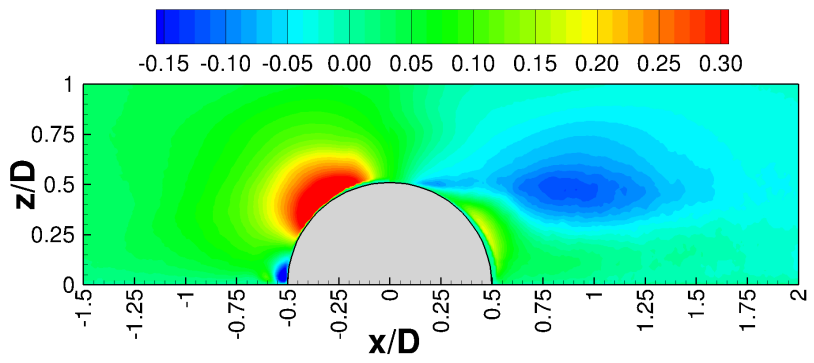

(d) $\bar{w} / U_{\infty}$ flexible

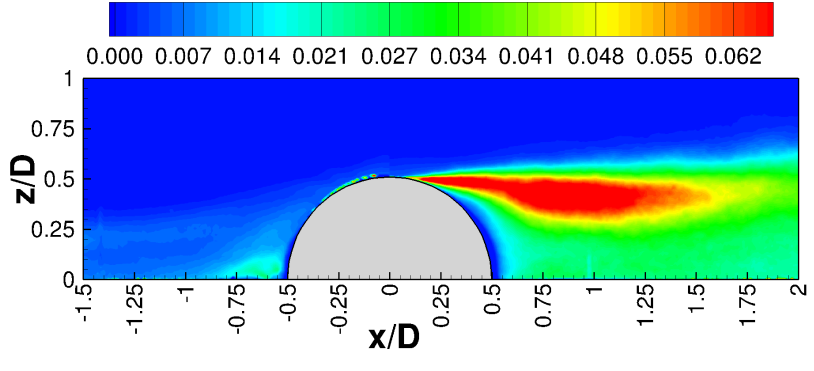

(f) $\overline{u^{\prime} u^{\prime}} / U_{\infty}^{2}$ flexible

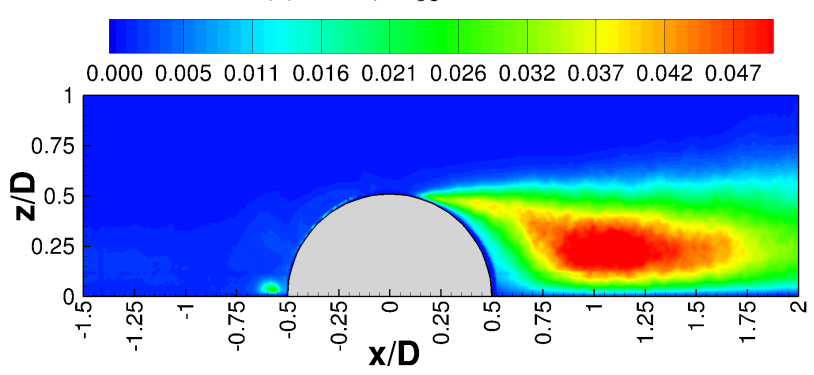

(h) $\overline{w^{\prime} w^{\prime}} / U_{\infty}^{2}$ flexible

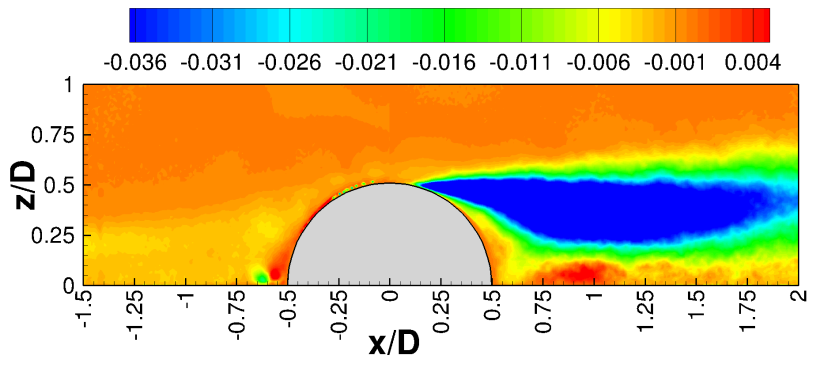

(j) $\overline{u^{\prime} w^{\prime}} / U_{\infty}^{2}$ flexible

Figure 9: Comparison of the time-averaged flow field characteristics of the rigid and the flexible hemisphere in the symmetry $x-z-$ plane at $y / D=0$ at $\operatorname{Re}=75,000$. 


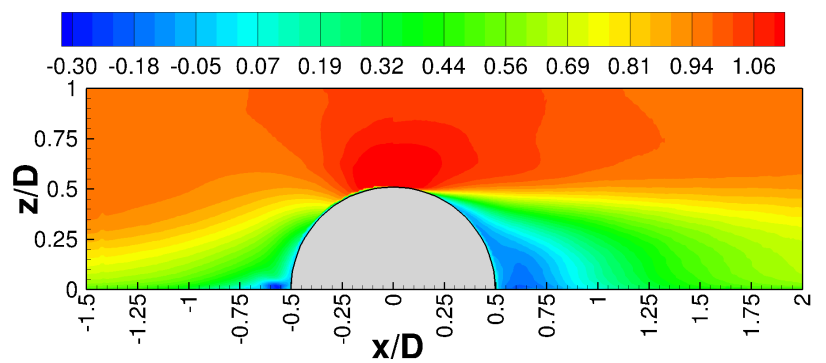

(a) $\bar{u} / U_{\infty}$ rigid

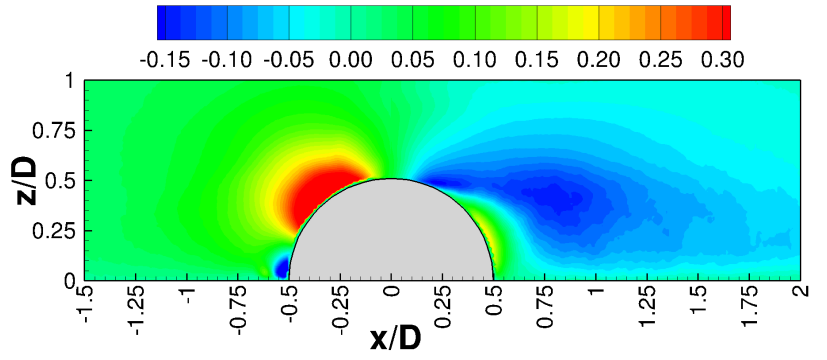

(c) $\bar{w} / U_{\infty}$ rigid

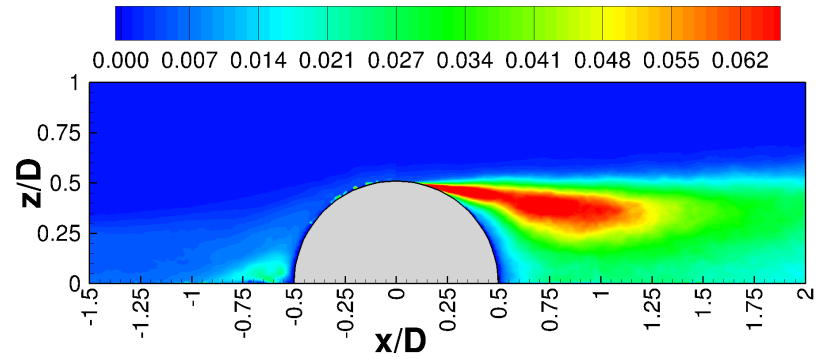

(e) $\overline{u^{\prime} u^{\prime}} / U_{\infty}^{2}$ rigid

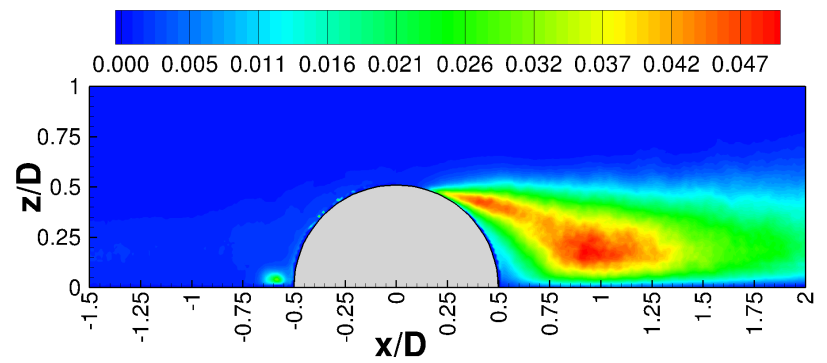

(g) $\overline{w^{\prime} w^{\prime}} / U_{\infty}^{2}$ rigid

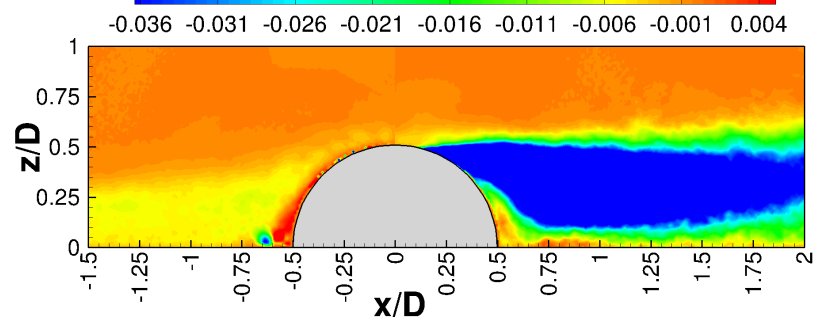

(i) $\overline{u^{\prime} w^{\prime}} / U_{\infty}^{2}$ rigid

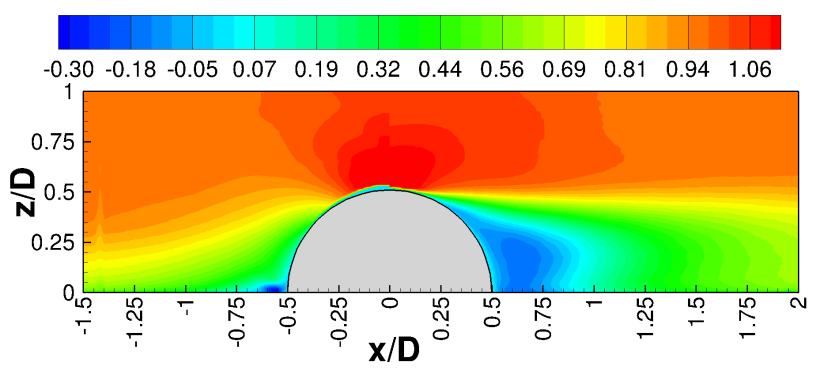

(b) $\bar{u} / U_{\infty}$ flexible

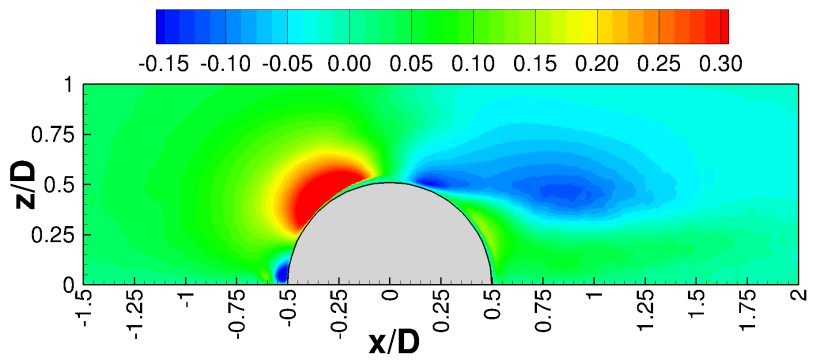

(d) $\bar{w} / U_{\infty}$ flexible

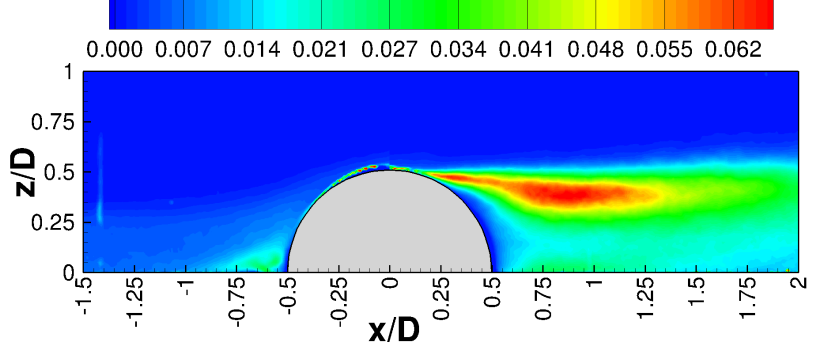

(f) $\overline{u^{\prime} u^{\prime}} / U_{\infty}^{2}$ flexible

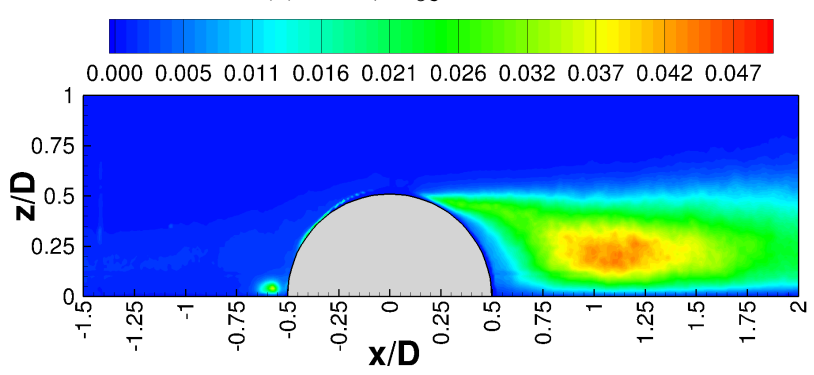

(h) $\overline{w^{\prime} w^{\prime}} / U_{\infty}^{2}$ flexible

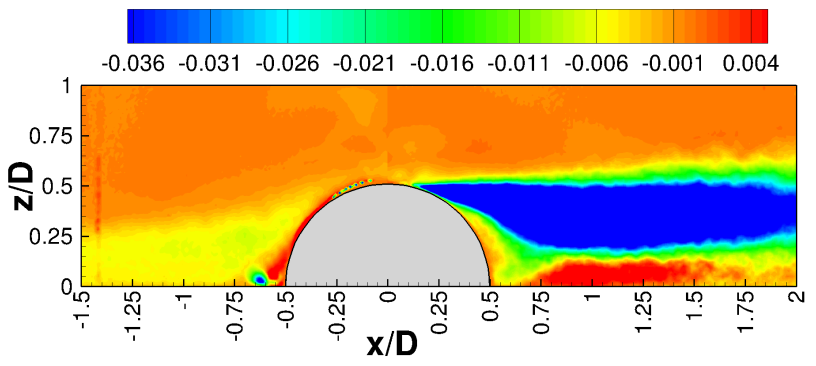

(j) $\overline{u^{\prime} w^{\prime}} / U_{\infty}^{2}$ flexible

Figure 10: Comparison of the time-averaged flow field characteristics of the rigid and the flexible hemisphere in the symmetry $x-z$-plane at $y / D=0$ at $\operatorname{Re}=100,000$. 


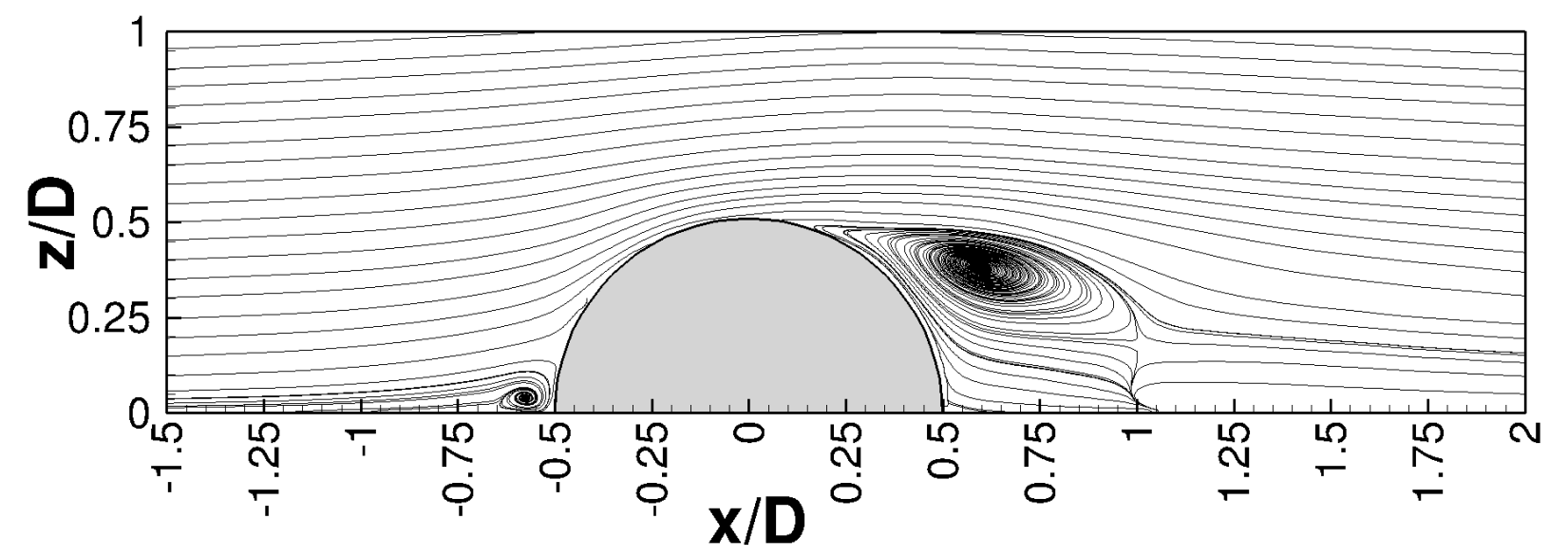

(a) rigid

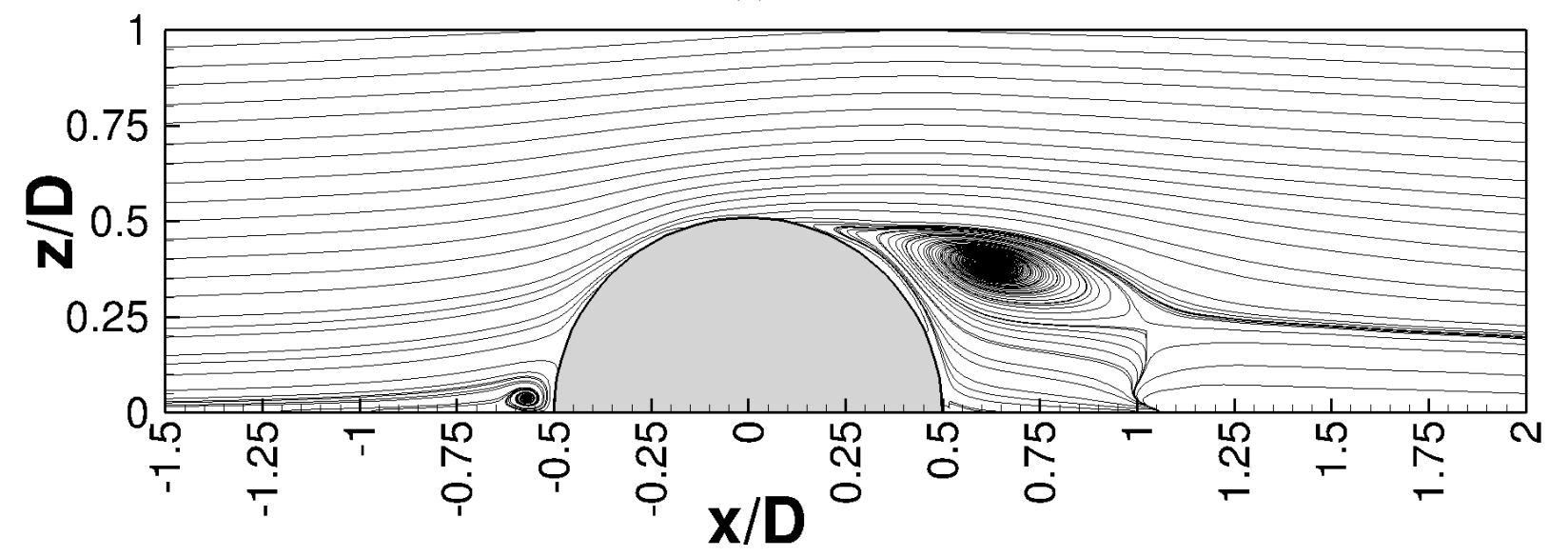

(b) flexible

Figure 11: Comparison of the time-averaged streamlines between the rigid and the flexible hemisphere at $\mathrm{Re}=50,000$.

a time-averaged global deformation superimposed by the oscillations of the structure mainly caused by the difference between the unsteady surface pressure distribution and the applied gauge pressure. Furthermore, an altered Reynolds stress distribution is observed in the wake. It is assumed that this reduction is caused by the energy transfer from the flow field to the flexible structure leading to attenuated fluctuations. To underline this hypothesis, a closer look at the unsteady and time-averaged structure displacements of the flexible hemisphere is presented in Section 4.3.

All previously described changes of the time-averaged velocity field are additionally highlighted by streamline plots in Figs. $11-13$. These illustrate the characteristic changes of the flow field for an increasing streamwise velocity and thus Reynolds number. The main reason for the changes observed is the effect of a delayed separation found for increasing Re. Since transition to turbulence moves upstream with increasing Reynolds number, the turbulent boundary layer can longer resist the adverse pressure gradient. Consequently, the separation line is noticeably shifted downstream (see Table 4) leading to a smaller recirculation region in the wake of the hemisphere. Thus, the recirculation on the lee-side of the hemisphere is the most affected area. In case of the rigid hemisphere with increasing Re number the center of the recirculation moves closer to the surface of the body, while the overall size decreases slightly resulting from the changing location of the separation point that is shifted towards the back of the rigid body as visible in Figs. 11(a), 12(a) and 13(a). The recirculation behind the flexible hemisphere exhibits 


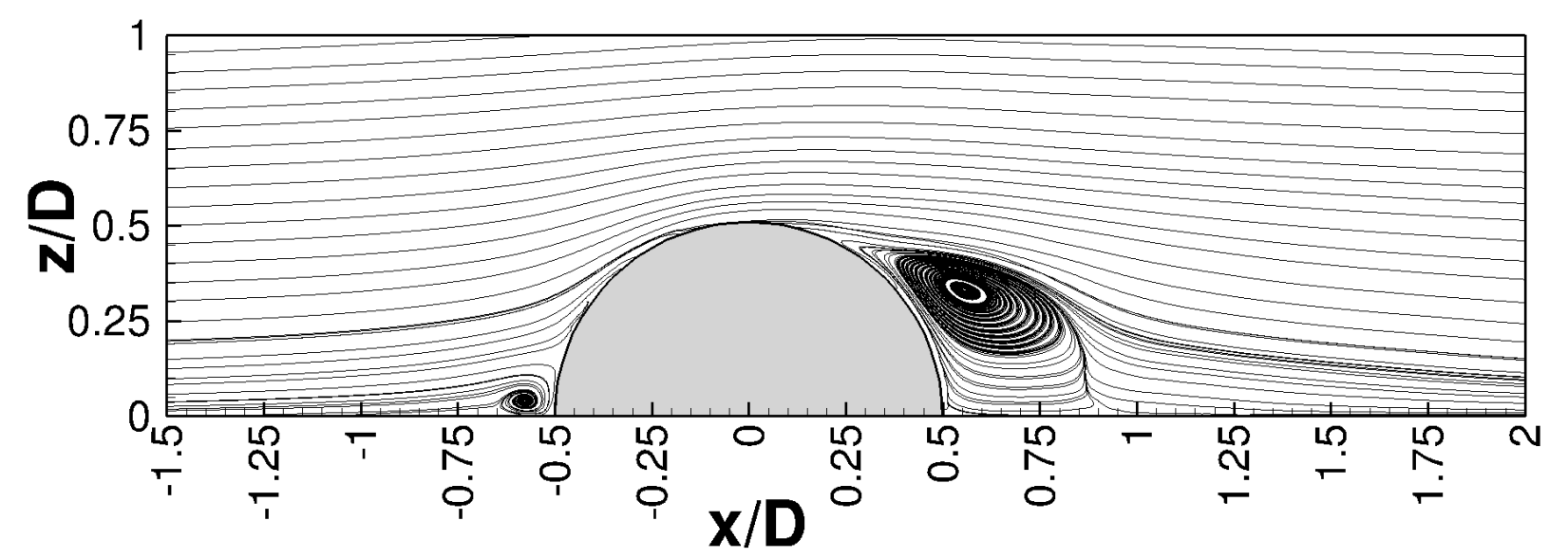

(a) rigid

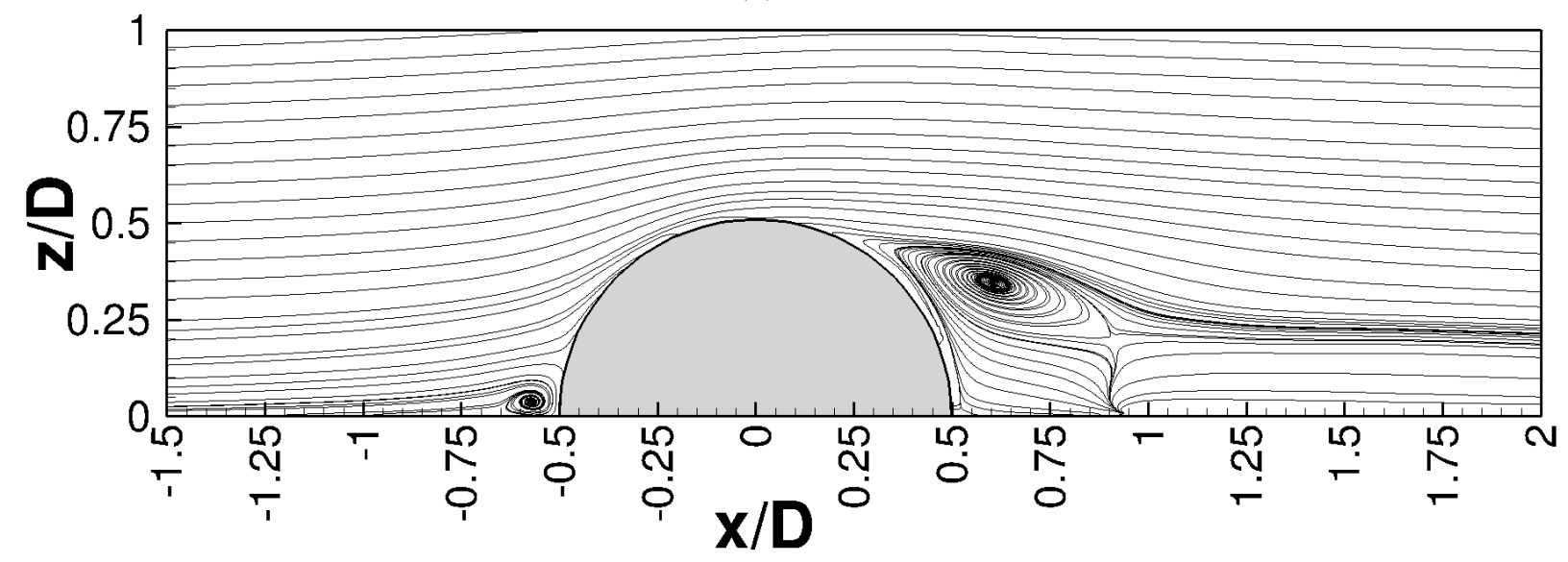

(b) flexible

Figure 12: Comparison of the time-averaged streamlines between the rigid and the flexible hemisphere at $\mathrm{Re}=75,000$.

noticeably different changes in its shape. It decreases in size at higher Re. Also the center of the recirculation moves away from the surface visible for $\mathrm{Re}=100,000$ in Fig. 13(b) from $x / D_{\text {rigid }}=0.56$ to $x / D_{\text {flexible }}=0.63$. Note that the other flow characteristics are summarized in Table 4.

An interesting detail is the progression of the dividing streamline throughout the wake. For the rigid case the flow passes the hemisphere and gradually moves towards the ground. This effect is significantly increased at higher Reynolds numbers. Contrary to this observation, the progression of the dividing streamline for the flexible hemisphere is oriented parallel to the ground throughout the entire wake without major changes in height. As a result the reattachment point is located further downstream compared to the rigid hemisphere visible for $\operatorname{Re}=75,000$ in Fig. 12. Finally, the horseshoe vortex in front of the hemisphere shows no larger variation for either case or Reynolds number.

In summary, comparing the time-averaged flow fields around the flexible and the rigid hemisphere at each Re separately, the largest deviations are found for the highest Reynolds number considered. As will be shown next this observation can be attributed to the largest timeaveraged deformations and the largest fluctuations of the structure measured for this case. 


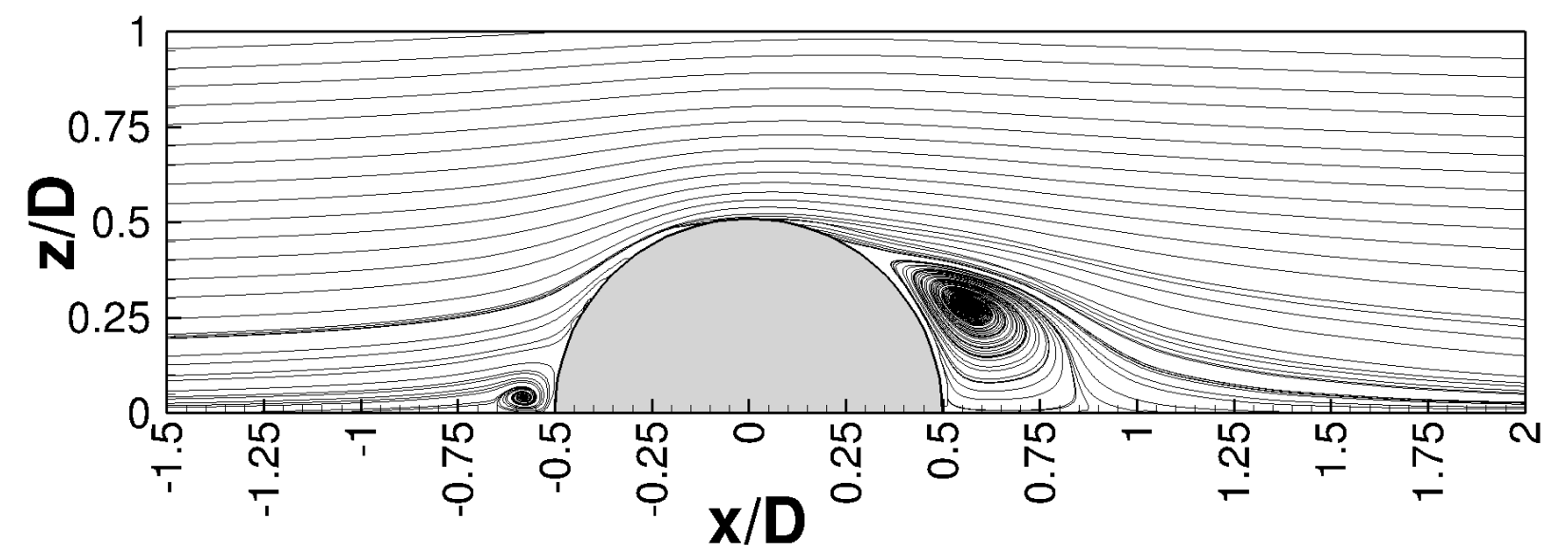

(a) rigid

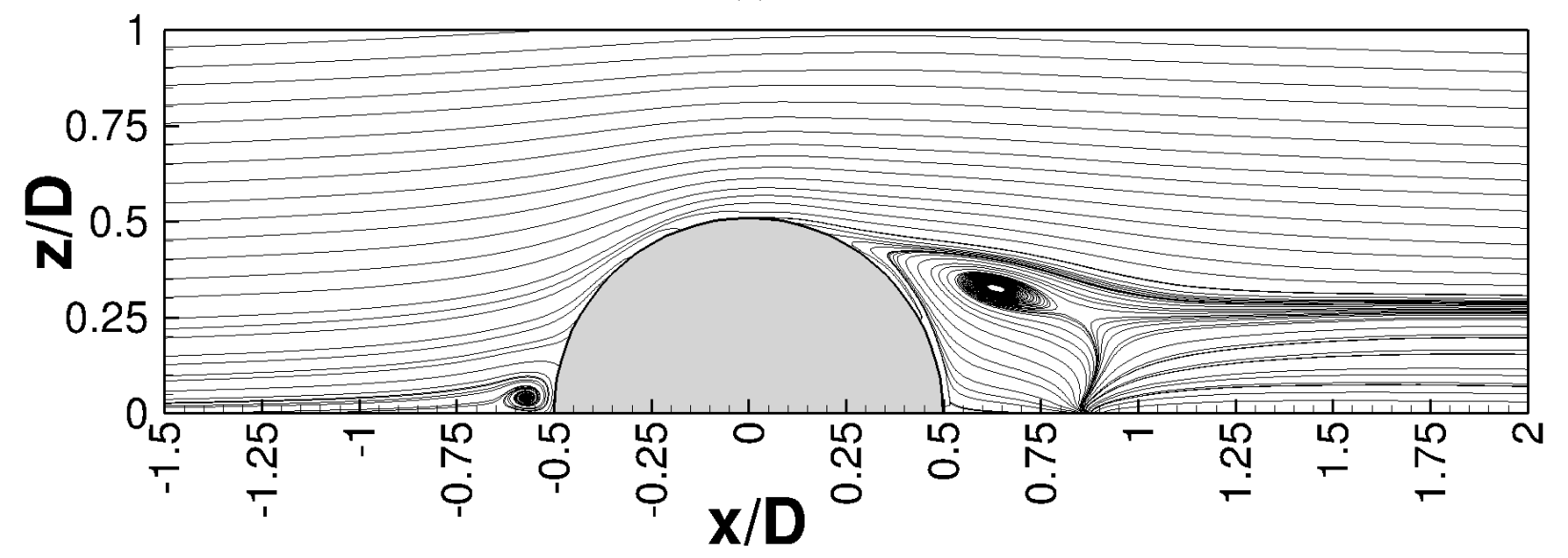

(b) flexible

Figure 13: Comparison of the time-averaged streamlines between the rigid and the flexible hemisphere at $\operatorname{Re}=100,000$.

Table 4: Comparison between the rigid and flexible case of characteristic flow field positions.

\begin{tabular}{|c|c|c|c|c|c|c|}
\hline \multirow{2}{*}{ Flow Characteristics } & \multicolumn{6}{|c|}{ Reynolds number } \\
\cline { 2 - 7 } & \multicolumn{2}{|c|}{$\mathbf{5 0 , 0 0 0}$} & \multicolumn{2}{|c|}{$\mathbf{7 5 , 0 0 0}$} & \multicolumn{2}{c|}{$\mathbf{1 0 0 , 0 0 0}$} \\
\cline { 2 - 7 } & rigid & flexible & rigid & flexible & rigid & flexible \\
\hline center of recirculation $x / D$ & 0.59 & 0.63 & 0.55 & 0.61 & 0.56 & 0.63 \\
separation from body $\varphi$ & $88^{\circ}$ & $79^{\circ}$ & $79^{\circ}$ & $73^{\circ}$ & $59^{\circ}$ & $63^{\circ}$ \\
reattachment point $x / D$ & 1.05 & 1.06 & 0.93 & 0.93 & 0.87 & 0.87 \\
\hline
\end{tabular}

\subsection{Structure response to wind loads}

This paragraph presents the time-averaged and unsteady results of the structure measurements. First, the time-averaged deformations and the standard deviations of the structure oscillation in the wake region are discussed. These are used to characterize the global deformation of the flexible structure as well as the oscillation behavior. Afterwards, the power spectra of the displacement components of each monitoring point are used to describe the impact of the unsteady turbulent flow on the structure excitations.

\subsubsection{Time-averaged deformation characteristics}

In order to analyze the time-averaged deformations caused by the fluid forces, a line element at the rear side in the symmetry plane is extracted from the DIC measurements. This line 
intersects with the chosen monitoring points starting at E15 and ending at the apex. The mean deformations are evaluated based on 5000 profiles at each Reynolds number. The results are given in Fig. 14.

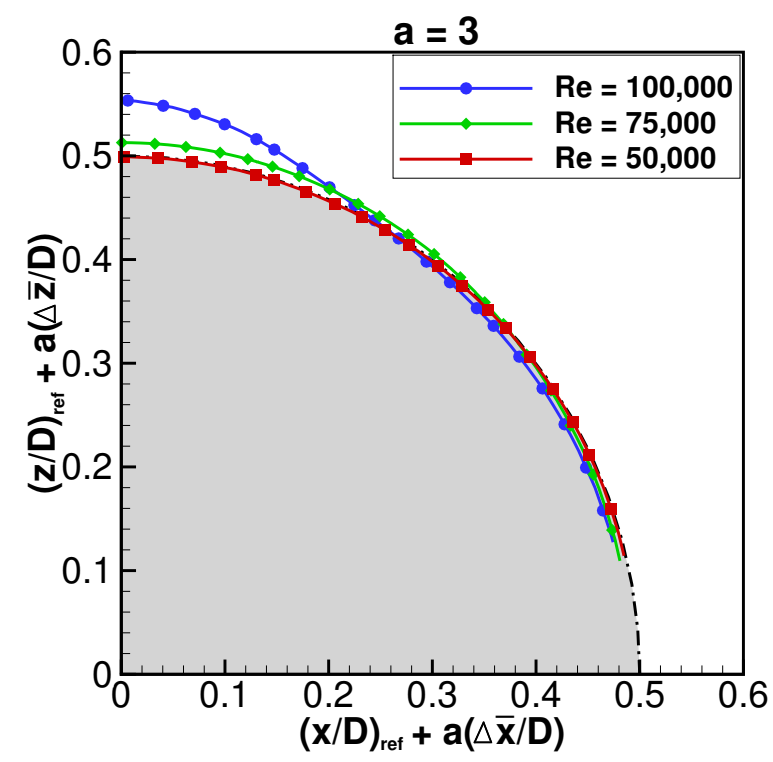

Figure 14: Comparison of the time-averaged deformations of the flexible hemisphere measured in the symmetry plane in the wake region $15^{\circ} \leq \varphi \leq 90^{\circ}$ (E15 - apex). A magnification factor of $a=3$ is applied to the average displacements $\Delta \bar{x} / D$ and $\Delta \bar{z} / D$ and added to the reference configuration to highlight the differences in shape.

To highlight the resulting deformation, a magnification factor of $a=3$ is chosen to increase the visibility of the mean displacements. This is done by adding the magnified mean displacements in dimensionless form $(a \Delta \bar{x} / D$ and $a \Delta \bar{z} / D)$ to the undeformed hemisphere. The deformed state at $\mathrm{Re}=50,000$ does not differ significantly from the reference case (rigid hemisphere). A slight deformation is present at $\operatorname{Re}=75,000$, mostly in the upper region. The largest difference is found at $R e=100,000$. Here, the area around the apex significantly moved upwards. This is caused by the increased pressure difference between the applied gauge pressure and the outer flow due to the accelerated velocity at the top of the hemisphere. The extracted profiles are furthermore used to evaluate the fluctuations of the structure. This is achieved by taking the standard deviations $\left(S_{x} / D\right.$ and $\left.S_{z} / D\right)$ of the displacements $\Delta x / D$ and $\Delta z / D$ as a measure of the fluctuations. The results are presented in Fig. 15. The fluctuations are plotted over their respective location referring to their angular position $\left(90^{\circ}\right.$ denotes the apex).

Figure 15(a) displays the fluctuations $S_{x} / D$ of the streamwise displacements. At $\operatorname{Re}=50,000$ the maximum fluctuations appear in a broad region between $37^{\circ} \leq \varphi \leq 55^{\circ}$. With increasing Reynolds number the region of maximal fluctuations gradually narrows and is shifted towards the ground floor. The strongest fluctuations $\left(S_{x} / D=1.75 \times 10^{-3}\right)$ are present at $\varphi=22^{\circ}$ for $\operatorname{Re}=100,000$. These fluctuations are caused by the vortex shedding occurring at the lower lateral sides of the hemisphere. This shedding process was described in detail for a rigid hemisphere at $\mathrm{Re}=50,000$ in Wood et al. (2016). When the Reynolds number increases, the frequencies of the vortex shedding increases, too. At $R e=100,000$ some frequencies related to the symmetric vortex shedding phenomenon come closer to dominant eigenfrequencies of the flexible structure, which leads to a stronger deformation of the hemisphere.

The wall-normal displacements depicted in Fig. 15(b) show the opposite behavior: In this case the magnitude of the movement of the membrane decreases when approaching angular positions close to the ground. In this region the vertical oscillations of the structure are very 


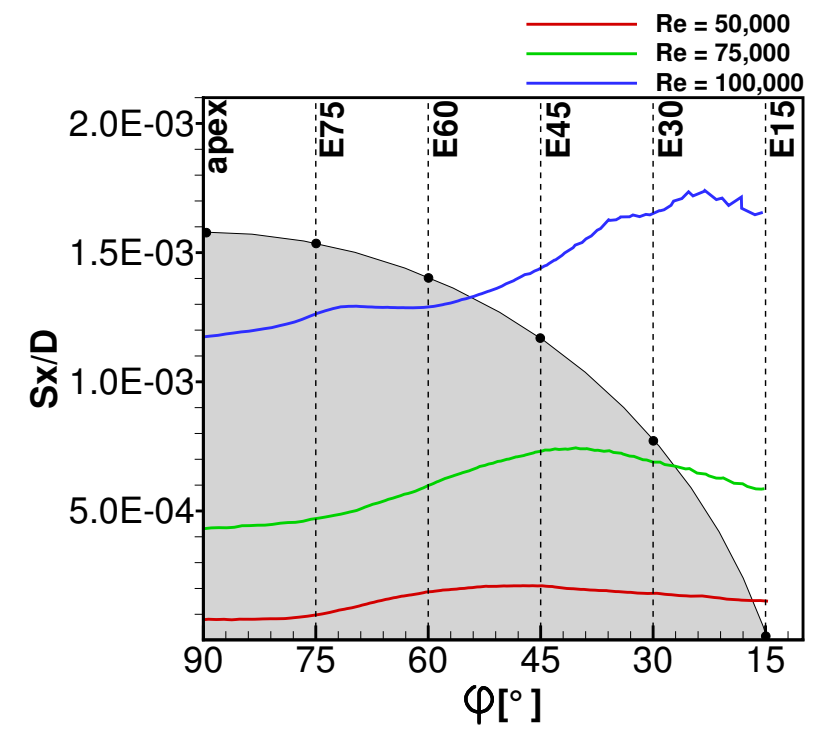

(a)

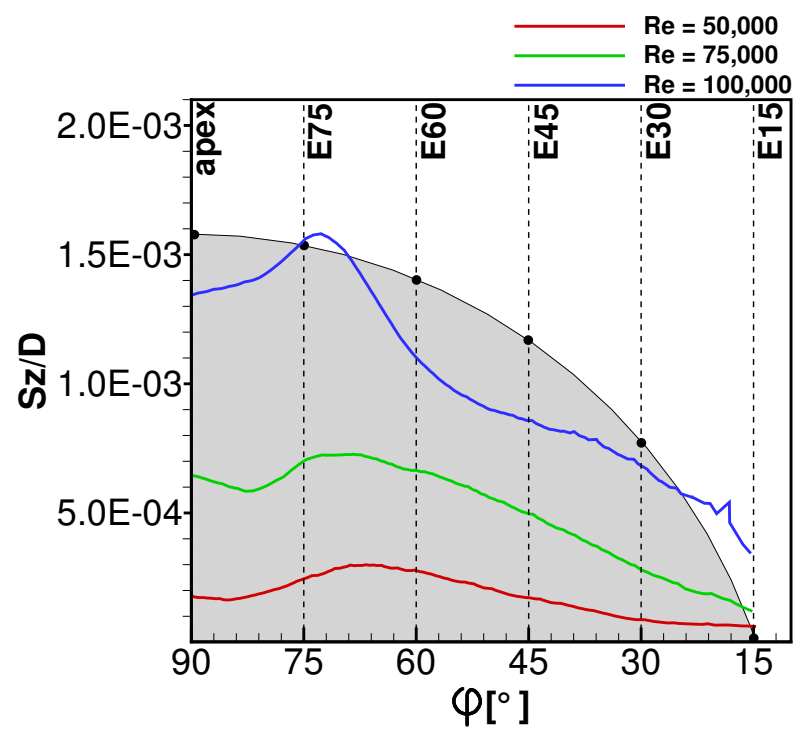

(b)

Figure 15: Comparison of the standard deviations $S_{x} / D$ and $S_{z} / D$ of the corresponding displacements.

small compared to the streamwise direction. The maximum fluctuations $\left(S_{z} / D=1.65 \times 10^{-3}\right)$ of the wall-normal component occur at $\varphi=72^{\circ}$ at $\operatorname{Re}=100,000$. At the same location a local maximum of $S_{x} / D$ can be detected. These observations are related to strong excitations induced by the roll-up of large vortices in the shear layer developing shortly after the separation line due to the Kelvin-Helmholtz instability.

\subsubsection{Unsteady structure excitations}

Before the unsteady structural behavior of the flexible hemisphere is discussed in detail, the three-dimensional deformations taken from the measurements at $\mathrm{Re}=100,000$ are presented in Fig. 16. The high-speed video data show complex deformation patterns that are linked to different excitation sources: The oncoming boundary layer is described as a superposition of a time-averaged profile and turbulent fluctuations. The time-averaged profile generates a constant load $\bar{F}$ on the structure, whereas the turbulent fluctuations impinging on the flexible structure lead to a fluctuating structural load $F^{\prime}$. In the classification of excitation sources by Naudascher and Rockwell (1994) these are related to the extraneous induced excitation (EIE). Based on the simple equation by Morison et al. (1950), a rough approximation of $\bar{F}$ and $F^{\prime}$ can be achieved showing that $F^{\prime}$ is about one order of magnitude less than $\bar{F}$. Therefore, these incoming turbulent fluctuations have a limited impact on the global dynamic deformation of the flexible hemisphere and no dominant frequency was found to be related to the fluctuations. Approximately at the prime meridian of the hemisphere the flow separates and a complex vortex shedding occurs. The different vortex shedding phenomena were investigated in a preliminary study based on the rigid hemisphere (Wood et al., 2016). These complex vortex sheddings are responsible for the observed time-dependent large deformations of the flexible hemisphere visible in the depicted time series. These vortex-induced vibrations (VIV) take the large part of the dynamic behavior of the coupled problem. The involved frequencies are discussed in detail in this section. In the presented time series of images (Fig. 16) the chosen reference configuration at $t^{*}=U_{\infty} t_{0} / D=0$ is an arbitrarily deformed state instead of the inflated hemisphere without flow. This procedure is chosen in order to visualize the detailed deformation patterns on the surface of the hemisphere. It leads to relative displacements since the offset between the usually applied static reference configuration $\Omega_{\text {cal }}$ (see Appendix A.2) and the loaded case during the 


\section{relative displacements $[\mathrm{mm}]$}
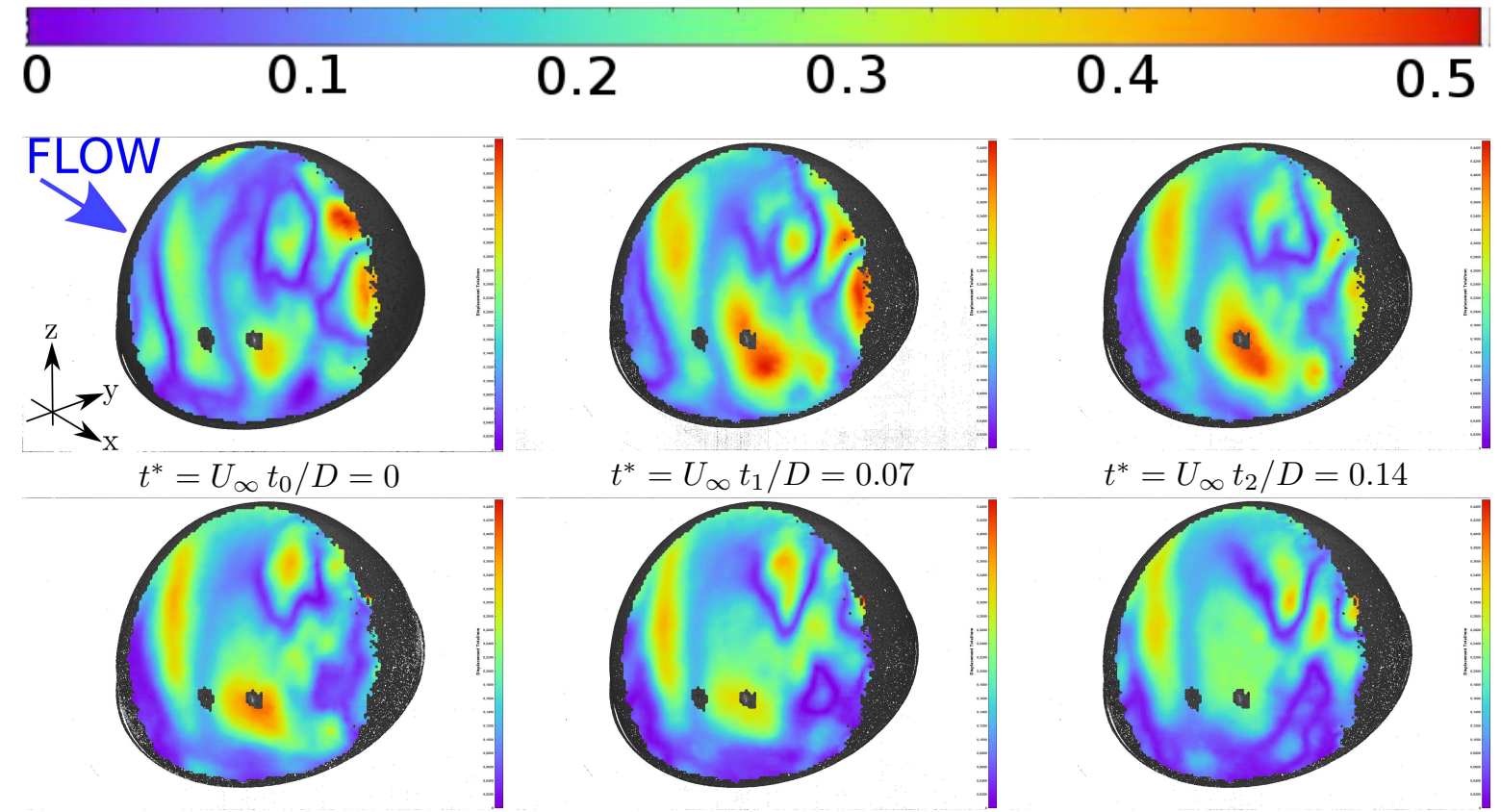

$t^{*}=U_{\infty} t_{3} / D=0.20$

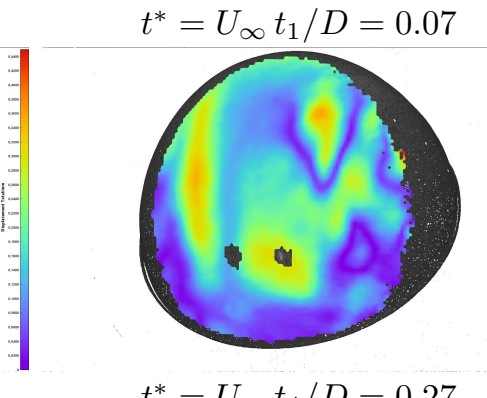

$t^{*}=U_{\infty} t_{2} / D=0.14$
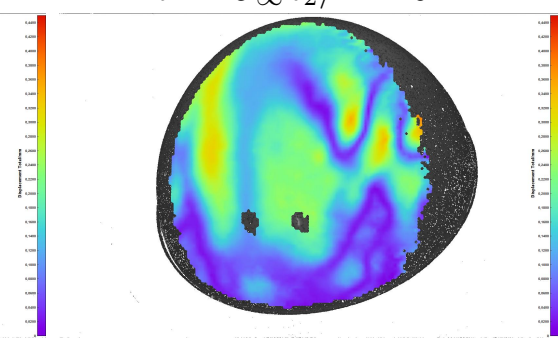

$t^{*}=U_{\infty} t_{5} / D=0.34$

Figure 16: Unsteady deformation behavior of the flexible hemisphere at $\operatorname{Re}=100,000$ (Wood and Breuer, 2017).

measurement is neglected. The two large dark spots are caused by reflections on the structure due to the applied light source leading to uncorrelated data.

Focusing again on the wake line, Fig. 17 depicts the power spectral density (PSD) of the structure deformations at each monitoring point (E15, E30, E45, E60, E75 and apex). The spectra are calculated from the displacements $\Delta x, \Delta y$ and $\Delta z$ for each Reynolds number.

Case $R e=50,000$

At $\operatorname{Re}=50,000$ the corresponding spectra exhibit relatively low amplitudes due to the small deformations as depicted in Figs. 17(a) - (c). The major part of the spectral power is found in the $\Delta x$ (streamwise) and $\Delta z$ (wall-normal) components. The strongest streamwise and wall-normal oscillations are found at the monitoring point E60 at a frequency of $35 \mathrm{~Hz}$ which is associated with the natural frequency $f_{n}^{1}$ (see Table 3). The distribution of the streamwise displacements $\Delta x$ is similar at all points except for the apex. A wide peak is located around $9.5 \mathrm{~Hz}$ (Strouhal number $\mathrm{St}=f D / U_{\infty}=0.27$ ) between $8.1 \mathrm{~Hz}$ and $10.9 \mathrm{~Hz}$ corresponding to a range of Strouhal numbers between 0.24 and 0.32 . This peak is connected with the arc-type symmetric vortex shedding process behind the hemisphere described in various publications on hemisphere flows (Manhart, 1998; Tavakol et al., 2015; Wood et al., 2016) and reported to have similar Strouhal numbers. In addition, a major peak at $40 \mathrm{~Hz}$ is observed followed by a sudden drop until a second large peak at $102 \mathrm{~Hz}$ is observed. The lateral displacements $\Delta y$, which are connected to a strong movement due to cross-flow, reveal a significant amplitude at $5.5 \mathrm{~Hz}$. This corresponds to a Strouhal number of $\mathrm{St}=0.16$ and is associated with the asymmetric von Kármán shedding process at the lateral sides of the hemisphere. This shedding type was also noticed with a similar Strouhal number in the previously mentioned publications on hemisphere flows (Manhart, 1998; Tavakol et al., 2015; Wood et al., 2016). Furthermore, a Strouhal number of $\mathrm{St}=0.40$ is found for all displacements. This is due to a strong roll-up of the shear layer 


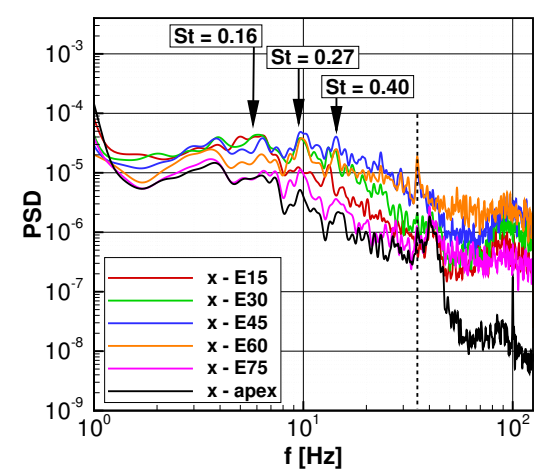

(a) PSD $\Delta x(\mathrm{Re}=50,000)$

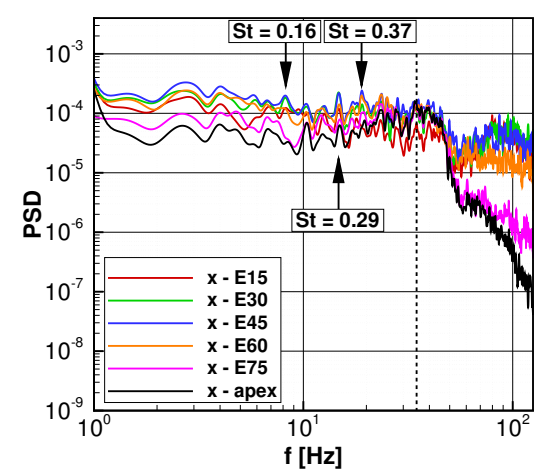

(d) PSD $\Delta x(\operatorname{Re}=75,000)$

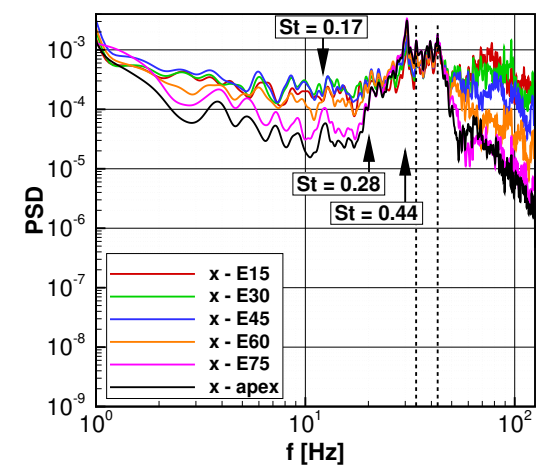

(g) PSD $\Delta x(\operatorname{Re}=100,000)$

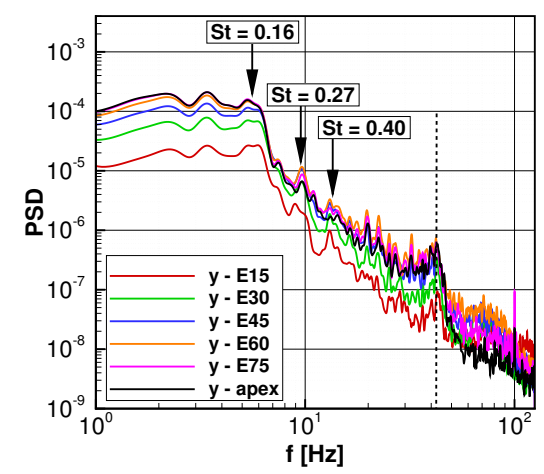

(b) PSD $\Delta y(\operatorname{Re}=50,000)$

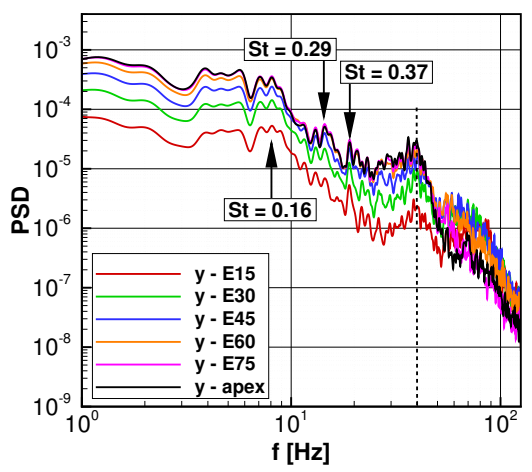

(e) $\mathrm{PSD} \Delta y(\mathrm{Re}=75,000)$

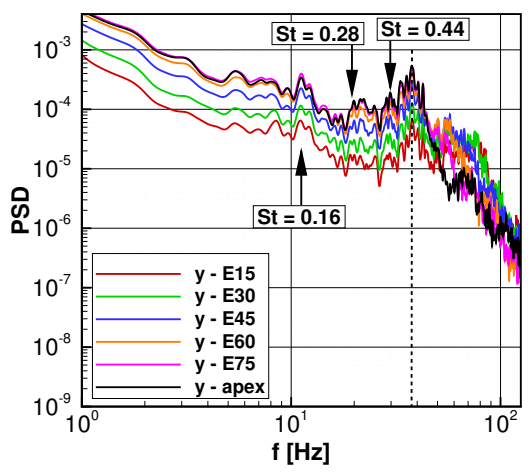

(h) PSD $\Delta y(\mathrm{Re}=100,000)$

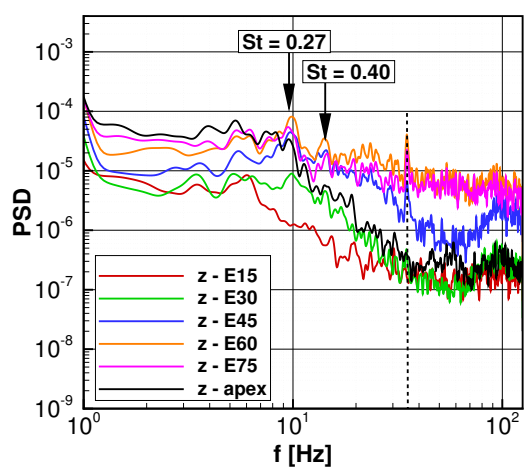

(c) PSD $\Delta z(\operatorname{Re}=50,000)$

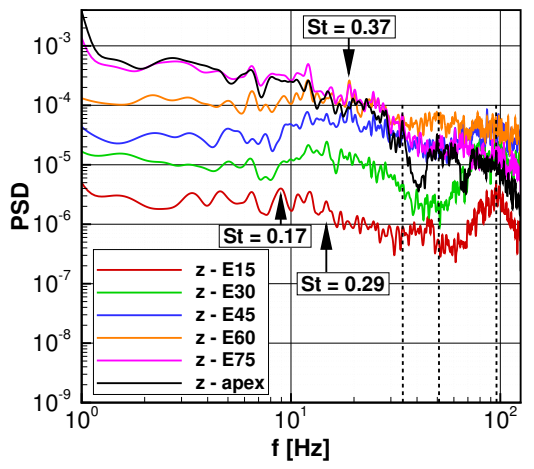

(f) $\mathrm{PSD} \Delta z(\mathrm{Re}=75,000)$

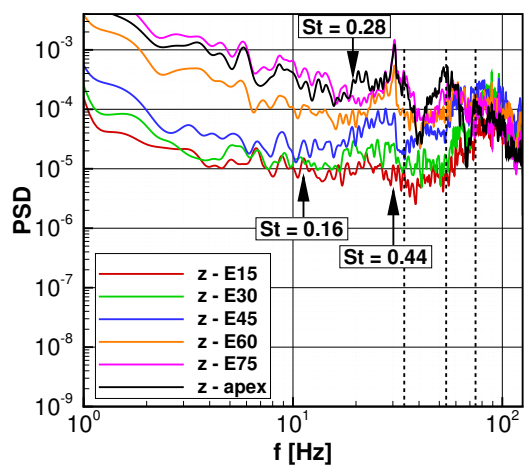

(i) $\operatorname{PSD} \Delta z(\operatorname{Re}=100,000)$

Figure 17: Power spectra of the deformations taken at the east-side monitoring points and at the apex of the flexible hemisphere.

forming large vortices exciting the structure. This is confirmed by an early study of Tamai et al. (1987) presenting the formation and shedding characteristics of vortices in the recirculation area of a rigid hemisphere. Especially the Kelvin-Helmholtz instability in the free shear layer contributes to this formation process as smaller vortices constantly merge until the resulting large vortex itself sheds from recirculation area. Manhart (1998) supported the existence of this shedding effect and gave a variation range between $0.2 \leq \mathrm{St} \leq 0.5$ for a rigid hemisphere at $\mathrm{Re}=150,000$. These large vortical structures were already visualized in Wood et al. (2016) as pressure fluctuations based on high-resolution large-eddy simulations at $\operatorname{Re}=50,000$.

The previously measured natural frequency $f_{n}^{2}=42 \mathrm{~Hz}$ of the hemisphere (see Table 3 ) is detected in the lateral component $\Delta y$ at each point. A noticeable aspect is found for the spectrum of the wall-normal component $\Delta z$. The maximum amplitude response is distributed almost constantly across the investigated frequency range. Especially the points E75 and E60, which are located close to the shear layer, show a nearly constant distribution over a wide 
frequency range with larger peaks at $35 \mathrm{~Hz}$ connected to $f_{n}^{1}$. At these points the oscillating movement of the flexible structure in vertical direction is obviously driving the other components since the corresponding amplitudes are largest. Additionally, the increase of the signal power at higher frequencies is interesting, which is clearly seen at E45.

Case $R e=75,000$

At $\operatorname{Re}=75,000$ the spectra for the streamwise displacements $\Delta x$ in Fig. 17(d) exhibit a more or less constant distribution in a narrow amplitude band until about $50 \mathrm{~Hz}$. After exceeding this frequency a decrease of the spectrum is found at the apex and E75. All other monitoring points show a similar behavior at higher frequencies with a broad peak between $50 \mathrm{~Hz} \leq f \leq 125 \mathrm{~Hz}$. Furthermore, several peaks are detectable in the Strouhal number range $0.21 \leq \mathrm{St} \leq 0.33$ and are related to the symmetric vortex shedding. The lateral movement $\Delta y$ at $\mathrm{Re}=75,000$ is presented in Fig. 17(e). It shows a similar trend as for $\mathrm{Re}=50,000$ with overall higher amplitudes throughout the whole frequency range and a Strouhal number of $\mathrm{St}=0.16$ caused by the asymmetric von Kármán vortex shedding. A local maximum is found at $19 \mathrm{~Hz}$ presumably connected to the previously discussed shear layer roll-up corresponding to $\mathrm{St}=0.37$. Furthermore, higher peaks are found in the range $30 \mathrm{~Hz} \leq f \leq 100 \mathrm{~Hz}$, which are related to natural frequencies of the flexible structure. A notable issue is present for the vertical excitations in Fig. 17(f). For this case the apex movement reveals two prominent local minima at about $40 \mathrm{~Hz}$ and $70 \mathrm{~Hz}$ as well as a peak with a high amplitude at about $35 \mathrm{~Hz}$ connected to $f_{n}^{1}$. A uniformly distributed spectrum with comparable amplitudes especially for higher frequencies is seen for E60. Moreover, a similar behavior is found at the points E75 and E45. An interesting feature is the PSD distribution of the wall-normal movement at the monitoring point E15. Here the higher frequencies contain most of the energy. This peak is associated with the natural frequencies $f_{n}^{8-13}$ of the structure visible only for the wall-normal component $\Delta z$ in Fig. $7(\mathrm{~d})$ of the dynamic response test.

Case $R e=100,000$

The power spectra at $\mathrm{Re}=100,000$ are illustrated in Figs. 17(g) - (i). For the streamwise component $\Delta x$ a dramatic amplitude increase in the frequency range between $30 \mathrm{~Hz} \leq f \leq 50 \mathrm{~Hz}$ with the maximum peak at $30 \mathrm{~Hz}$ is observed leading to a Strouhal number of $\mathrm{St}=0.44$. Once again, this frequency is connected to a strong vortex formation in the recirculation area observable at every monitoring point with comparable amplitudes. Several remarkable peaks are observable in the range $34 \mathrm{~Hz} \leq f_{n} \leq 42 \mathrm{~Hz}$. As the formation and shedding frequencies of the fluid approach the natural eigenfrequencies of the structure $\left(f_{n}^{1}\right.$ and $\left.f_{n}^{2}\right)$, a narrow band with comparable amplitudes at every monitoring point is detected. Besides this, strong oscillations occur at higher frequencies at the points E15, E30 and E45. Similar to this observation, the lateral component $\Delta y$ shows a broad peak in the range $30 \mathrm{~Hz} \leq f \leq 42 \mathrm{~Hz}$ with a maximum amplitude at about $38 \mathrm{~Hz}$. Moreover, asymmetric von Kármán $(\mathrm{St}=0.16)$ and symmetric shedding $(0.27 \leq \mathrm{St} \leq 0.31)$ processes are visible. The wall-normal component exhibits qualitatively the same distribution as seen at $\mathrm{Re}=75,000$ with a sharp peak at $30 \mathrm{~Hz}$ for the apex, E75 and E60, which are located close to the fluctuating shear layer. For these monitoring points distinctive frequency peaks are separated by local minima at $40 \mathrm{~Hz}, 70 \mathrm{~Hz}$ and $80 \mathrm{~Hz}$. The decrease of the PSD at $40 \mathrm{~Hz}$ is not detected in the streamwise or lateral movement due to a strong excitation of the natural frequency $f_{n}^{2}=42 \mathrm{~Hz}$.

\section{Influence of the Reynolds number on the structure excitations}

A characteristic vortex formation is detected between $0.37 \leq \mathrm{St} \leq 0.44$ connected to the roll-up of the shear layer. Furthermore, two vortex sheddings are observed: An asymmetric von 
Kármán vortex shedding at $\mathrm{St}=0.16$ and a symmetric one in the range $0.21 \leq \mathrm{St} \leq 0.33$. The dominant eigenfrequencies of the flexible structure are found for $30 \mathrm{~Hz} \leq f_{n}^{i} \leq 120 \mathrm{~Hz}$. By increasing the Reynolds number, the frequencies of the vortex formation and sheddings increase. At approximately $\operatorname{Re} \approx 100,000$, the vortex formation frequency starts to overlap with the lowest eigenfrequencies causing an amplification of the structural deformations.

In general, the characteristics of the presented spectra at each monitoring point follow the distribution of the standard deviations in Figs. 15(a) and (b) discussed previously for the investigated Re number range with restrictions to the $\Delta x$ and $\Delta z$ displacements. High amplitudes in the spectrum at specific monitoring points are connected to large standard deviations at the same location which are an indicator for an amplified structural movement. After the analysis of the structural deformations of the flexible hemisphere the results are now compared to CTA measurements of the flow in the near-wall region close to the hemisphere in order to investigate the fluid-structure interaction.

\subsection{Fluid-structure interactions of the flexible hemisphere}

\subsubsection{Correlation between velocity fluctuations and structure oscillations in the wake}

To correlate the structure deformation measurements with the fluid measurements, additional experiments using a constant-temperature probe are carried out. The PIV data in Section 4.2 reveal a significant difference between the Reynolds stresses in the wake of the rigid and the flexible case, especially close to the surface of each hemisphere. This effect is strongest at $\operatorname{Re}=100,000$. Hence, throughout the rest of the study this specific Reynolds number is used to identify the characteristic behavior of the flexible hemisphere in turbulent flow.

First, the link between the structural movement and the fluid field is discussed. This is achieved by comparing the previously presented spectra of the oscillating structure based on the DIC measurements with the velocity spectra recorded by a 1D CTA probe close to each monitoring point at a distance of about $2 \mathrm{~mm}$ from the surface as depicted in Fig. 18. This distance is mainly necessary to avoid damage of the fragile probe due to collisions with the oscillating membrane. It is expected that the frequencies measured by the DIC system are also present in the CTA data.

In the following, the correlations between the velocity and the displacement spectra are shown in Fig. 19. Each plot presents the velocity spectrum as a black line while the deformation

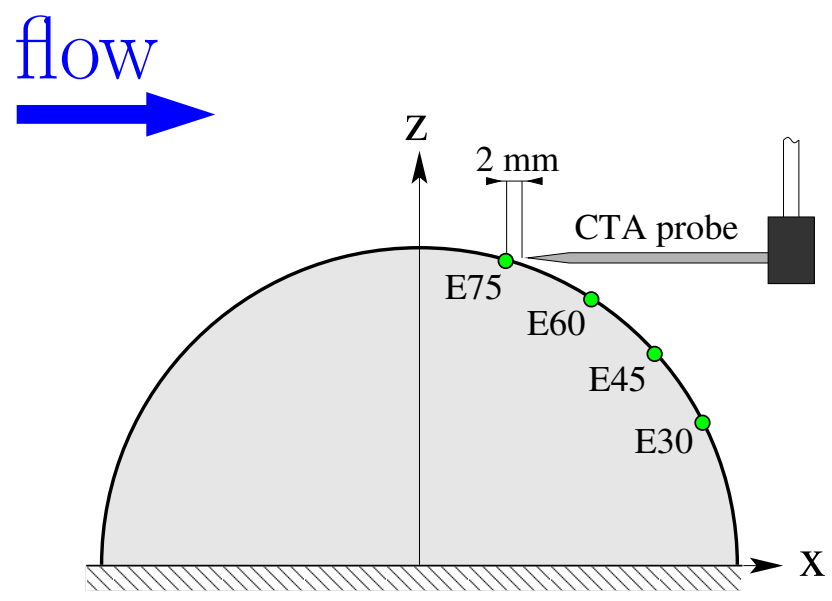

(a) Schematic close-wall CTA setup.

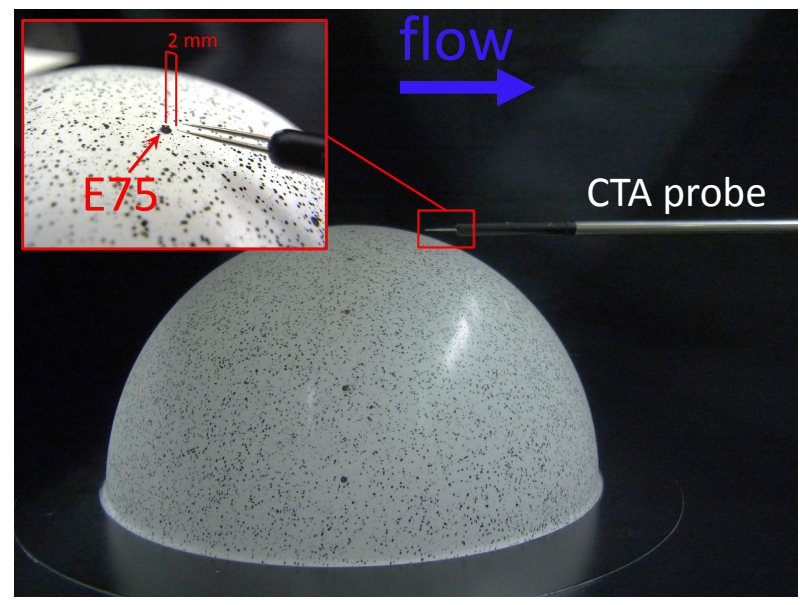

(b) Actual setup with flexible hemisphere.

Figure 18: Schematic representation and actual CTA setup for the close-wall velocity fluctuation measurements. 


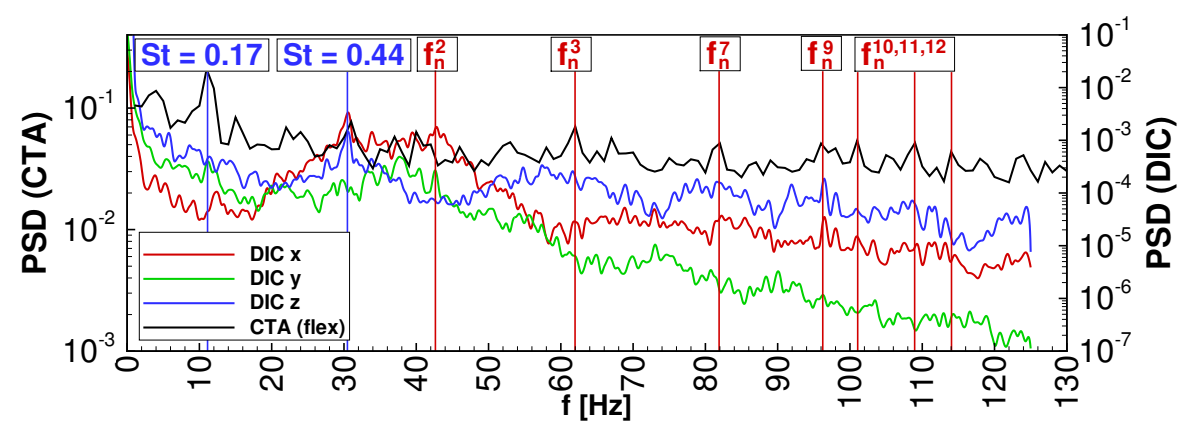

(a) PSD close to surface point E75.

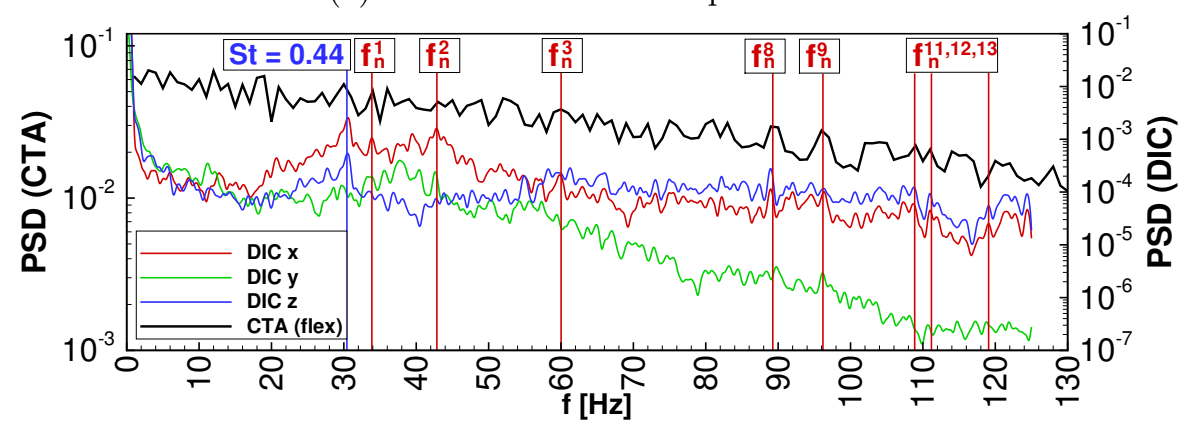

(b) PSD close to surface point E60.

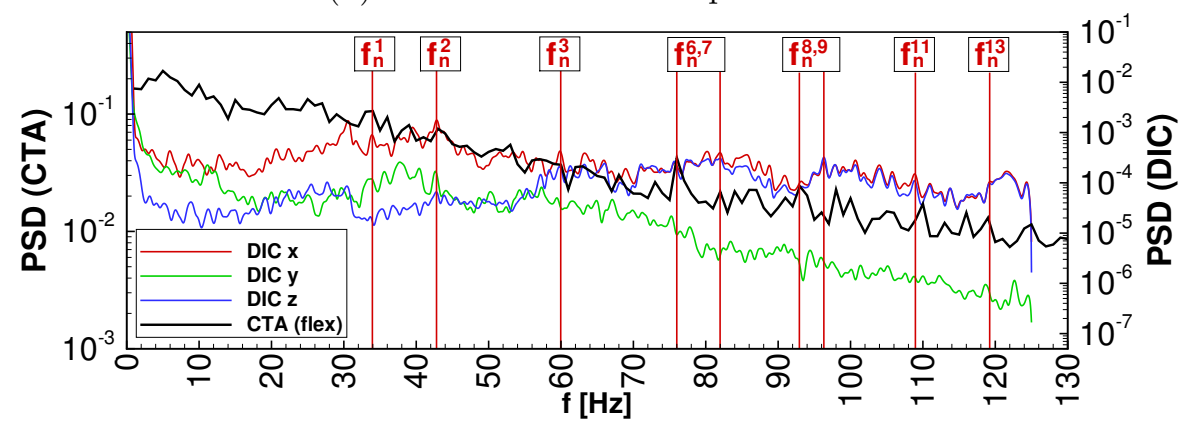

(c) PSD close to surface point E45.

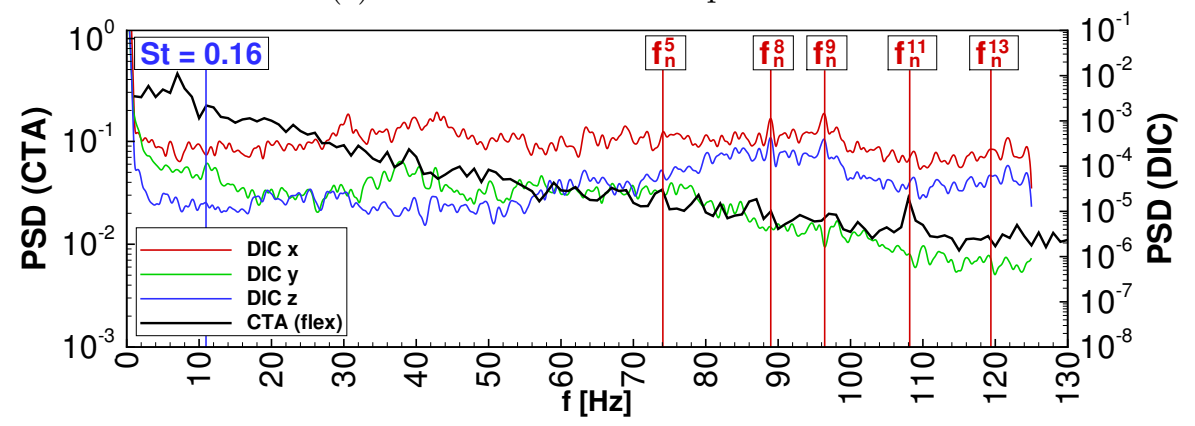

(d) PSD close to surface point E30.

Figure 19: Frequency spectra of the velocity fluctuations close to the surface at the chosen monitoring points (E75, E60, E45 and E30) in the wake region and the frequency spectra of the displacements of the flexible hemisphere at $\operatorname{Re}=100,000$.

spectra are colored red $(\Delta x)$, green $(\Delta y)$ and blue $(\Delta z)$. The diagrams are used to identify similarities in the spectral domain between the close-wall fluid velocities and the movement of the flexible wall. Furthermore, the data are applied to examine the physical source of interesting frequencies, such as vortex shedding or natural frequencies of the membrane.

Figure 19(a) depicts the spectra at E75. The CTA measurement records a peak at about $11.5 \mathrm{~Hz}$. This frequency is also visible in the DIC measurements, especially in the $\Delta y$-component. It is associated with the quasi-periodic von Kármán vortex shedding that occurs at the lateral 
sides of the hemisphere. It corresponds to a Strouhal number of St $=f D / U_{\infty} \approx 0.17$ and is in good agreement with previous studies of a rigid hemisphere (Wood et al., 2016). Additionally, a strong connection between the fluctuations of the fluid and the excitation of the membrane is present at a frequency of $30 \mathrm{~Hz}(\mathrm{St}=0.44)$. As discussed above, this effect is driven by the formation of vortices in the recirculation area resulting from the roll-up of the separated shear layer. This link between the spectra of the fluid and the structure measurements, especially for the monitoring points close to the shear layer (E75 and E60), indicates a dominant shedding frequency. Besides this, several natural eigenfrequencies of the structure are influencing the flow field at the measuring points of the CTA probe, which are also visible in the signal of the DIC data as strong membrane excitations. Similar spectra for the other monitoring points are presented in Figs. 19(b) - (d). The 1D CTA probe is aligned with the spanwise $y$-direction, which means that the streamwise and wall-normal velocity components contribute to the measured velocity signal, i.e., $\overline{u^{\prime} u^{\prime}}$ and $\overline{w^{\prime} w^{\prime}}$. Moreover, contrary to E75 and E60 the amplitude of the velocity components for E45 and E30 (in the symmetry plane and in this region of the recirculation area) is rather small. Therefore, the peaks associated with the von Kármán vortex shedding $\mathrm{St}=0.16$, the symmetric vortex shedding $(0.21 \leq \mathrm{St} \leq 0.33)$ and the vortex formation $(\mathrm{St}=0.44)$ are not so pronounced for these monitoring points in the CTA data. Nevertheless, the velocities close to the hemisphere can be linked to the structural behavior since significant frequencies of both spectra (CTA and DIC) match well.

Second, it is assumed that the kinetic energy from the fluid is partially transferred to the flexible structure leading to a reduction of the velocity fluctuations in the wake. To investigate this issue, a comparison of the velocity spectra close to the wall between the rigid and the flexible model is carried out. By comparing the power of the signal for both cases at the same location, it is possible to quantify this effect. A frequency band-width analysis in Fig. 20 presents the flow characteristics at four chosen points (E75, E60, E45 and E30) in the wake. Due to measurement restrictions in the setup, fluid data at point E15 could not be measured. A bar plot illustration is applied to visualize the differences of the amplitudes in the power spectra across the whole measurement range $(2-125 \mathrm{~Hz})$. The data of the rigid hemisphere are plotted behind the ones of the flexible model. This is mainly done because the amplitudes of the solid hemisphere are generally larger, except for point E75. To analyze this trend quantitatively in more detail, the spectra are divided into four frequency bands. Each band is analyzed by cumulating its power content by the Signal Power ${ }_{j}=\sum_{i}\left(\mathrm{PSD}_{i} \cdot \Delta f_{i}\right), f_{i} \in$ band $_{j}$. The subdivision of the whole data set into individual bands is useful to avoid the biasing of the power spectrum towards the largest amplitudes. The procedure allows to characterize the velocity fluctuations in specific frequency ranges. The gained cumulative results of every band are plotted in Fig. 20(e). As expected the largest amount of the signal power is contained in the first band where a continuous decrease is observed towards higher bands. The points E60, E45 and E30 follow the same trend: The power content of the signal of the rigid hemisphere is generally larger than for the flexible case. This supports the assumption that the kinetic energy of the fluid is partially transferred to the structure lowering the velocity fluctuations on the lee-side of the object.

A similar effect was observed in a direct numerical simulation carried out by Shen et al. (2003) studying the turbulent flow over a smooth wavy wall undergoing transverse motion in the form of streamwise traveling waves. Close to the wall a significant reduction of the turbulence intensity and turbulent shear stresses was detected compared to a solid wall. However, in contrary to this, Rosti and Brandt (2017) found in their numerical simulations an increase of the Reynolds stresses in the near-wall region of a hyper-elastic viscous wall. Following this, Rojratsirikul et al. $(2009,2010)$ compared a rigid with a membranous wing in a Reynolds number range between 


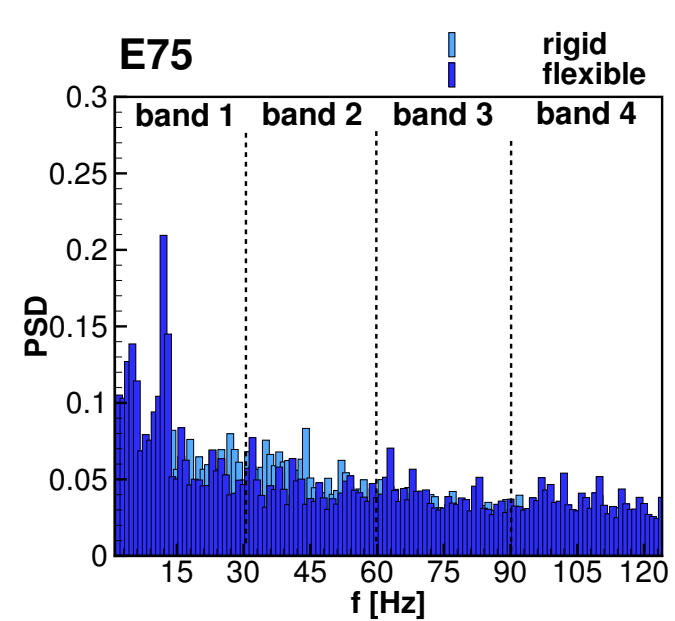

(a) PSD E75 rigid vs. flexible

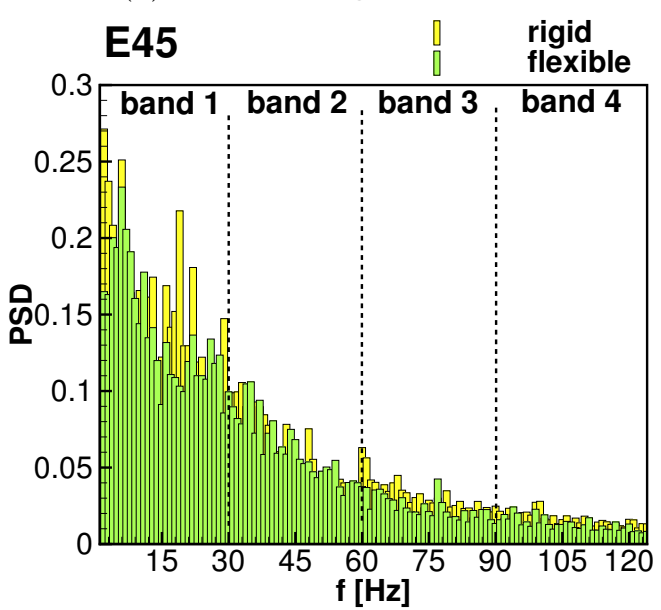

(c) PSD E45 rigid vs. flexible

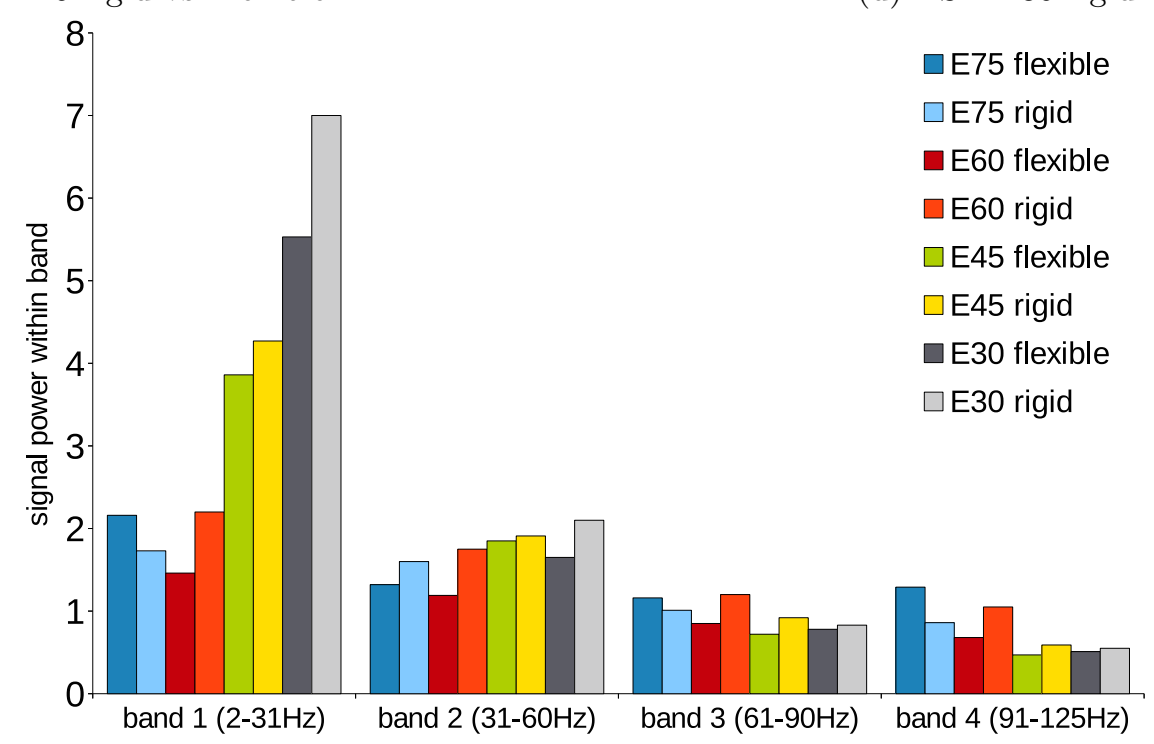

(e) Cumulative power content of each band.

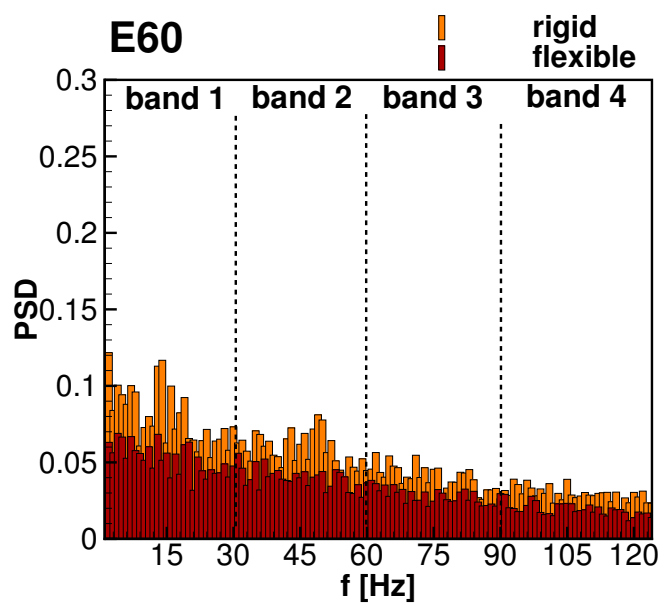

(b) PSD E60 rigid vs. flexible

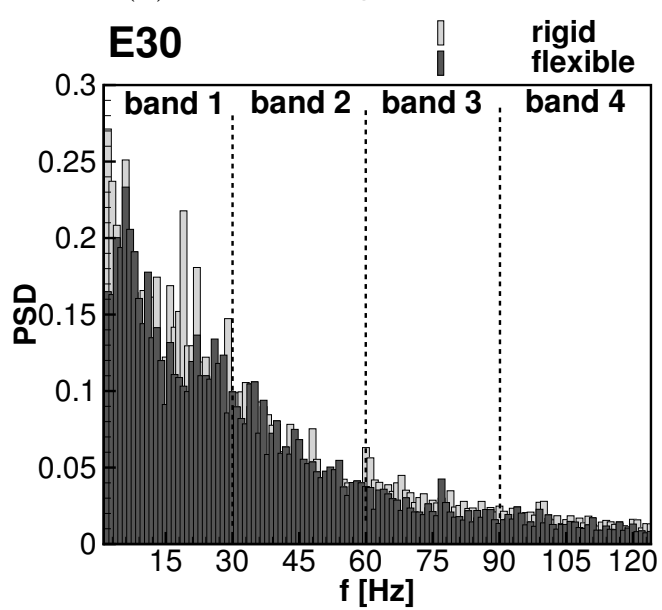

(d) PSD E30 rigid vs. flexible

$$
\begin{aligned}
& \square E 75 \text { flexible } \\
& \square E 75 \text { rigid } \\
& \square \text { E60 flexible } \\
& \square E 60 \text { rigid } \\
& \square E 45 \text { flexible } \\
& \square E 45 \text { rigid } \\
& \square E 30 \text { flexible } \\
& \square E 30 \text { rigid }
\end{aligned}
$$

Figure 20: Comparison of the velocity spectra (CTA measurements) at points (E75, E60, E45, E30) close to the structure between the rigid and the flexible hemisphere including the decomposition of the spectra in 4 bands $(\operatorname{Re}=100,000)$. 
$\operatorname{Re}=53,100$ and 106,000 as outlined in the introduction. The membrane was made of silicone material to emulate the flexible characteristics of bat wings. One interesting finding showed that the membrane excitations resulted in increased Reynolds stresses on the upper side of the wing compared to the rigid wing. The flow separated later from the wing at higher angles of attack due to its flexibility. These seemingly contrary effects indicate a strong dependency on the problem considered. In case of the present air-inflated flexible hemisphere it is clearly seen that the velocity fluctuations in the wake region are reduced.

Interestingly, this behavior is not evident for point E75. At this location the power content of band 1,2 and 4 is larger compared to the rigid case. This can be explained by the different separation points of the flow that occur for each hemisphere. In case of the flexible hemisphere the separation in mean is further upstream near the apex. The CTA probe is already recording larger fluctuations due to the developing shear layer after the detachment of the flow from the surface. The flow around the rigid hemisphere separates at a location which in average lies further downstream. Thus, the shear layer is at an earlier development stage and therefore measures lower fluctuations.

In conclusion, with the exception of the point E75 close to the free shear layer, at all other monitoring points the flexible structure reduces the Reynolds stresses in the wake regime due to an energy transfer from the flow to the flexible structure.

\subsubsection{Influence of the approaching turbulent boundary layer on the structure excitations}

A comparison of the velocity spectra between the approaching turbulent boundary layer measured at the inlet of the test section at $x / D=-1.5$ and the corresponding velocity spectra of the hemisphere measurements in the wake are depicted in Fig. 21. The velocity spectra of the turbulent boundary layer were recorded at a vertical distance $z$ comparable in height with the monitoring points E30 and E45 for Re $=100,000$. These points were especially chosen following the assumption that significant frequencies would first of all occur within the log-law region (fully turbulent region) of the boundary layer. The plots do not reveal any dominant frequency

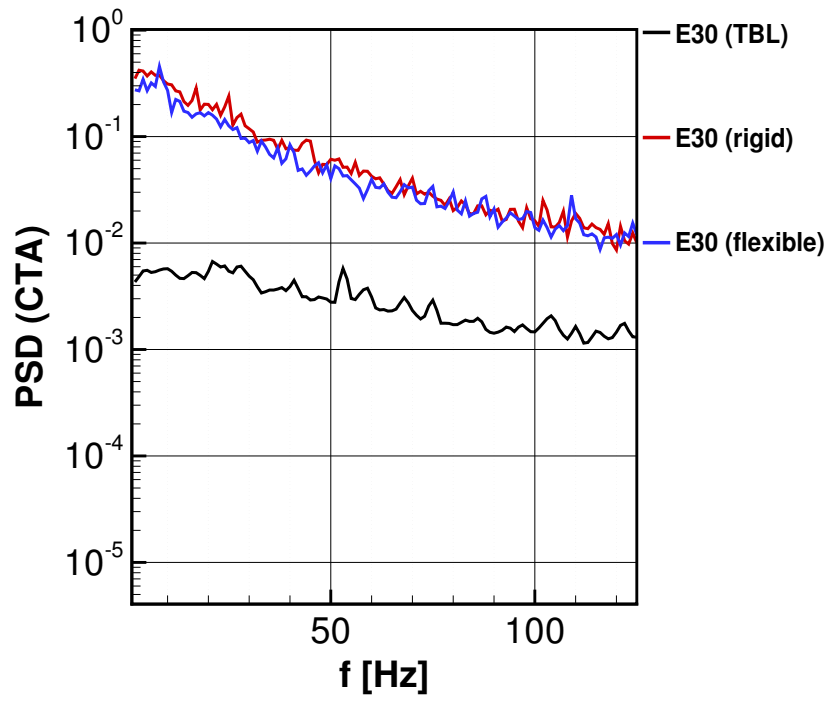

(a) E30

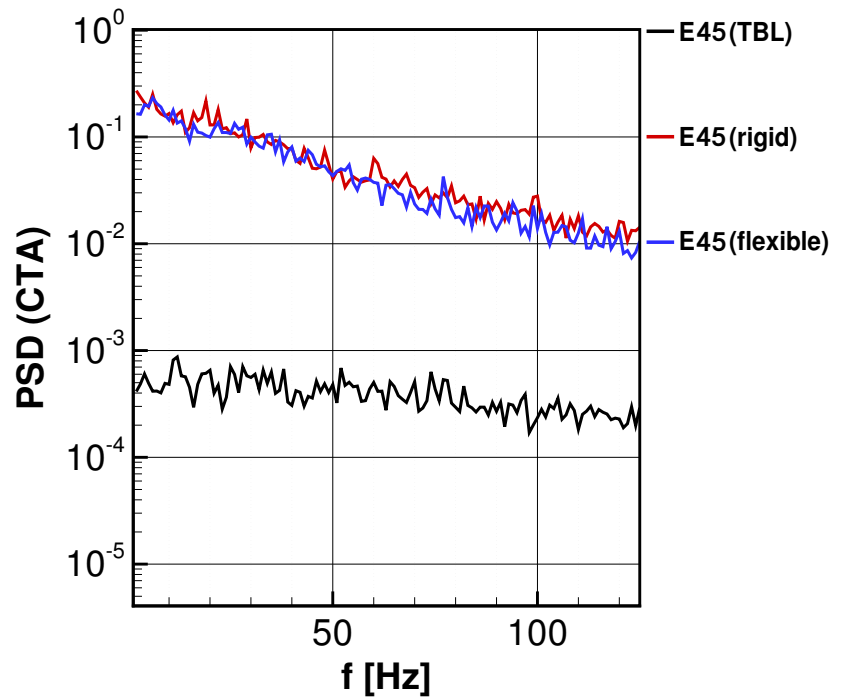

(b) E45

Figure 21: Comparison of the frequency spectra of the approaching turbulent boundary layer (TBL) of the empty channel with the measurements made for the rigid and the flexible hemisphere at the locations E30 and E45. 
peaks, which could be correlated to the spectra of the actual hemisphere measurements. If a critical frequency would be present in the approaching flow, it should be detected as a specific Strouhal number in the flow field or as a structure excitation in the DIC measurements. In both cases the rigid and the flexible hemisphere showed no global effects resulting directly from the turbulent boundary layer. Most of the turbulence of the oncoming flow is visible as short transient local deformations that are caused by vortical structures with strong vorticity impacting the front side of the flexible hemisphere. These events usually have no significant effect on the general oscillating behavior. Furthermore, the PSD values of the approaching boundary layer are one to two orders of magnitude smaller than the corresponding values past the hemisphere. As expected, the magnitude of the power spectrum decreases with the distance to the wall recognizable in Fig. 21(b).

\subsection{Limitations of the non-synchronized FSI measurements}

In the present setup the link between fluid and structure measurements is mainly based on the Reynolds stresses (fluid) and the standard deviations of the deformations (structure). According to this approach it is argued that the individually obtained data can be linked as long as a long-term time-dependency can be excluded, i.e., the collected data are statistically converged. Great care was taken to prove the reproducibility of the setup taking all measurement devices into account. To avoid this drawback of the present setup, fully synchronized measurements would be necessary making a direct relation between the fluid and the structure at each time step possible. Due to the problems of synchronized measurements discussed in Section 3.3.2, a fully coupled three-dimensional numerical FSI simulation using large-eddy simulation and a specialized structure solver for membrane elements is presently run in parallel (Breuer et al., 2016). Based on this complementary approach the presently missing link between both disciplines can hopefully be elucidated. Nevertheless, as a first step to better link fluid and structure measurements at the FSI interface, data along a line in the wake are considered. For this purpose, the correlation coefficient

$$
\phi_{x, y}=\frac{\operatorname{Cov}(x, y)}{\sqrt{\operatorname{Var}(x) \operatorname{Var}(y)}}=\frac{S_{x, y}^{2}}{S_{x} S_{y}}
$$

is used, where $S_{x y}^{2}$ is the co-variance and $S_{x}$ and $S_{y}$ are the standard deviations. The resulting values can only vary in the range $-1 \leq \phi_{x, y} \leq 1$, where $\phi_{x, y}= \pm 1$ denotes a perfect linear correlation of the observed variables, whereas $\phi_{x, y}=0$ indicates that there is no correlation. This approach has to be treated very carefully since the utilized data sets have to fulfill certain properties such as a normal distribution and the stated linear characteristics. In case of a nonlinear behavior between two variables (displacements $\Delta x, \Delta z$ or velocity components $u, w$ ) the correlation coefficient is either insufficient or useless. For the present case it is assumed that in the near-wall region the correlation coefficient shows a similar distribution when comparing the individual data of the fluid and structure measurements. In Fig. 22 the correlation coefficient of the DIC measurements of the wake profile and the Reynolds stresses taken from the PIV measurements close to the wall are compared.

Interestingly, the graphs of the correlation coefficients are comparable at $\operatorname{Re}=50,000$ and 75,000 showing a similar behavior for the velocity fluctuations and the structure oscillations. In case of $\operatorname{Re}=100,000$ there are still significant correlations between the variables of the individual fields (fluid and structure), but the graphs of the fluid and structure measurements show partially a contrary behavior. This might be a problem resulting from the spatial resolution of the 


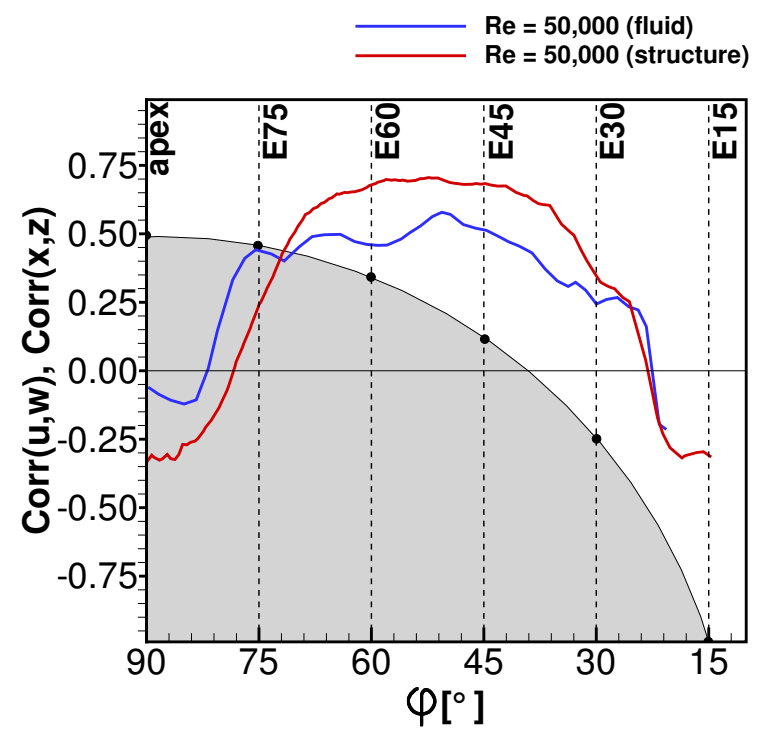

(a) $\operatorname{Re}=50,000$

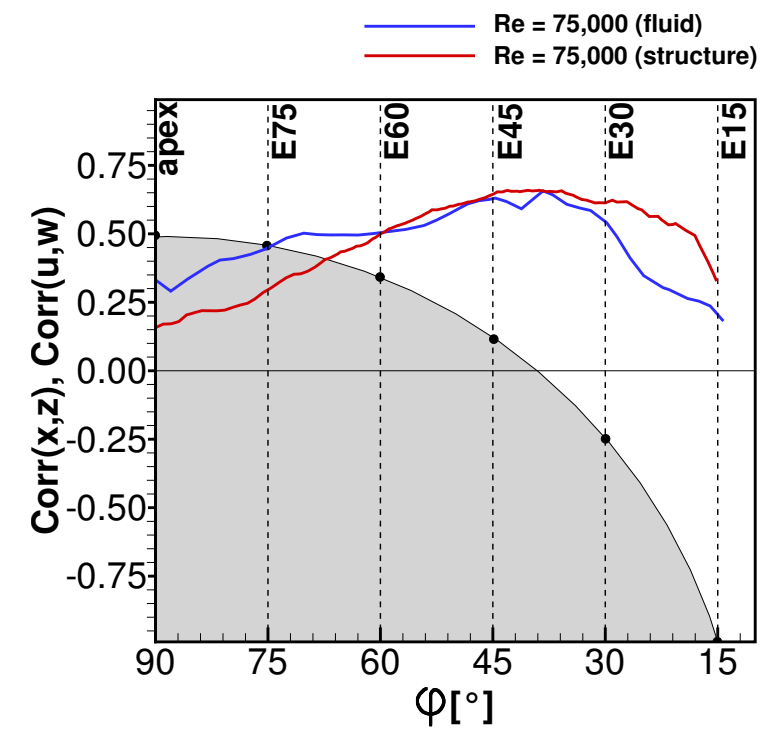

(b) $\operatorname{Re}=75,000$

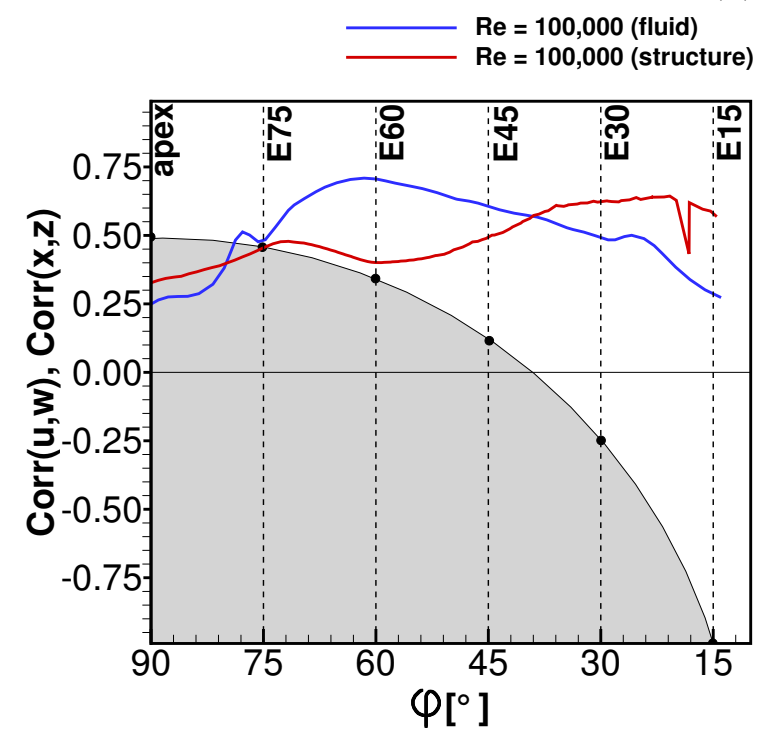

(c) $\operatorname{Re}=100,000$

Figure 22: Correlation coefficient used as statistical measure to evaluate the interaction mechanisms between the fluid and the structure. Values for the fluid are taken close to the wall and obtained by PIV measurements.

PIV system in the given setup. It is imaginable to find a more similar behavior between the structure and the fluid field when moving the camera system closer to the hemisphere and thus increasing the spatial resolution directly at the observed wake line. However, this approach has to be treated with caution since there could as well be a non-linear correlation between the variables which represents the physical properties of the flow far better.

An alternative approach to analyze the correlation between both fields can be achieved by determining the "mutual information" of the experimental data. The corresponding probability distribution of each data set is used as a measure for the degree of statistical dependency between the examined variables. This concept is not restricted to linear correlations and could lead to an improved understanding of the FSI phenomenon. A detailed review on this subject was given by Abarbanel et al. (1993) stating the applicability of mutual information theory on chaotic data taken from experimental measurements with reference to fluid dynamics, i.e., 
Journal of Fluids and Structures, vol. 80, pp. 405-440, (2018).

turbulence.

\section{Conclusions}

The present study examines the fluid-structure interaction of a thin-walled membranous hemisphere exposed to an artificially generated turbulent boundary layer at $\operatorname{Re}=50,000$, 75,000 and 100,000. The measurements are carried out in a wind tunnel, where the boundary layer is adjusted to exhibit minimum variations in the mean velocity profile and turbulence intensities. This boundary layer is a superposition of a classical mean turbulent velocity profile with proper fluctuations. These clearly defined inflow conditions offer the advantage of a straight forward transfer to complementary numerical simulations. The generated inflow profile does not contain strong transient variations of the velocity (gusts) and is therefore not comparable with natural wind characteristics. A silicone model of the flexible hemisphere is presented including all relevant model and material properties. To stabilize the model against the wind loads, the membranous hemisphere has to be pressurized. Furthermore, the flow around a rigid hemisphere is measured under identical conditions serving as reference.

Applying comprehensive high-spatially resolved two-dimensional PIV for the flow field and three-dimensional high-speed DIC measurements for the deformable membrane, the coupled phenomenon is studied. Both measurement techniques are used independently from each other due to their highly specialized specification range. Therefore, additional CTA measurements are conducted at specific monitoring points close to the surface in the wake of the hemisphere in order to find further links between the fluid flow and the structure deformations.

First, the time-averaged velocity fields around the rigid and the flexible hemisphere are investigated by comparing mean velocities and Reynolds stresses. With growing Reynolds number the differences between both cases increase. Here, the wall-normal velocity component and the distribution of the Reynolds stresses are significantly attenuated. The turbulent fluctuations in the wake region, especially close to the surface along the lee side of the flexible hemisphere, are lower than in the rigid case. The reason is found in the transfer of kinetic energy from the fluid to the deformable structure, which is stabilized by the gauge pressure. Furthermore, a comparison of the streamlines exhibit a remarkably different shape towards higher Reynolds numbers underlining the effects of the flexible structure on the mean flow.

Second, the time-averaged structure deformations are evaluated along a line in the wake restricting to the symmetry plane. The mean structure deformations alter visibly with increasing Reynolds number with the largest deflections found at $R e=100,000$. The standard deviations of the coordinates of the oscillating wake line are calculated as a measure for the structure fluctuations. As expected, the most significant displacements occur at $R e=100,000$. The largest values in wall-normal direction are observed close to the apex of the hemisphere. The structure oscillations in this region are the result of the separation and roll-up of the shear layer arising from the Kelvin-Helmholtz instability. As a subsequent step, the unsteady flow characteristics are studied at distinctive monitoring points located on the wake line. For this purpose, the power spectral density is evaluated based on the instantaneous structure measurements in order to identify significant frequencies that can be linked to their physical origin. Distinctive Strouhal numbers are found which are caused by different vortex shedding processes: an asymmetric von Kármán vortex street at St $=0.16$ and an arc-type symmetric vortex shedding in the range $0.21 \leq \mathrm{St} \leq 0.33$. Another interesting flow phenomenon is the formation of vortices in the recirculation area within a Strouhal number range of $0.37 \leq \mathrm{St} \leq 0.44$. With increasing Re the frequency of this last vortex formation process approaches the lowest natural frequencies of the flexible structure leading to amplified oscillations of the membrane. Additional CTA measurements concentrate at points close to the wall of the hemisphere. The resulting velocity fluctuations are linked to the measured structure oscillations. Besides the above mentioned 
vortex shedding phenomena a wide range of excited natural eigenfrequencies of the membranous hemisphere are detected in the spectra. Finally, the near-wall velocity spectra of the flexible hemisphere are compared with the rigid case in order to investigate the influences of the flexible wall on the flow field. A frequency band analysis reveals that the velocity fluctuations close to the surface are lower in case of the flexible structure supporting the initial assumption based on the PIV measurements. Consequently, energy is transferred from the flow field to the flexible structure resulting in a decrease of velocity fluctuations in the wake visible as an overall reduction in the Reynolds stresses.

Furthermore, the influence of the approaching turbulent boundary layer on the FSI phenomena was examined by comparing the frequency spectrum of the velocity fluctuations. The velocity fluctuations of the boundary layer of the empty channel flow were analyzed at two locations. These were compared to the velocity spectra in the wake of both hemispheres at $\operatorname{Re}=100,000$ to determine possible effects of the oncoming flow field on the structure excitation. As a result, the fluctuations of the approaching turbulent boundary layer have no significant influence on the structure excitation at the investigated points in the wake of the rigid and the flexible hemisphere.

This novel case of a pre-stressed membranous structure in turbulent flow still offers numerous open questions such as the three-dimensional phenomena important for this coupled problem which can be not answered with the help of the experimental data alone. Thus, further investigations based on fully coupled numerical simulations are carried out. These will be presented in a comprehensive follow-up paper.

\section{Acknowledgments}

The numerical part of the project is financially supported by the Deutsche Forschungsgemeinschaft under the contract number BR 1847/12-2. Furthermore, the authors want to thank Natalie Rauter (Department of Mechanical Engineering / Helmut Schmidt University Hamburg) for her assistance during the determination of the elastic behavior of the silicone material.

\section{Appendix A. Quality evaluation of the flexible model}

\section{Appendix A.1. Evaluation of the casting process}

The casting process was examined statistically to ensure the reproducibility of the flexible model (Fig. A.23). For this purpose, 26 models were produced using the same amount of silicone and hardener. An additional inhibitor (Wacker@ PT 88) was applied to 10 models which had an significant effect on the mass of the cured model. This is depicted in the histogram in Fig. A.23(a). The large gap in the histogram of the total mass distribution in the middle is caused by the inhibitor. As the curing time is delayed from 6 to 48 hours $\left(\Delta t_{\text {curing }}=42 \mathrm{~h}\right)$ the silicone can flow longer out of the casting form. This reduces the mass of the final model. Additionally, the inhibitor reduces the average thickness of the wall as shown in Fig. A.23(b). In conclusion, the present study was carried out with a membrane produced under the influence of the inhibitor with a total mass of $12.44 \mathrm{~g}$.

The thickness of the hemisphere was measured using three different devices. First, a micrometer with a precision of $0.005 \mathrm{~mm}$ was used. This technique is suitable for a rough estimate, since the deformation of the material has a significant influence on the measurement. Second, a contact-free method using a laser displacement sensor (Micro-Epsilon scanCONTROL 2750-100) was used to measure the thickness of a test hemisphere at arbitrary points. For this purpose, seven circular holes were inserted into the material (Fig. A.24(a)). The flexible model was 


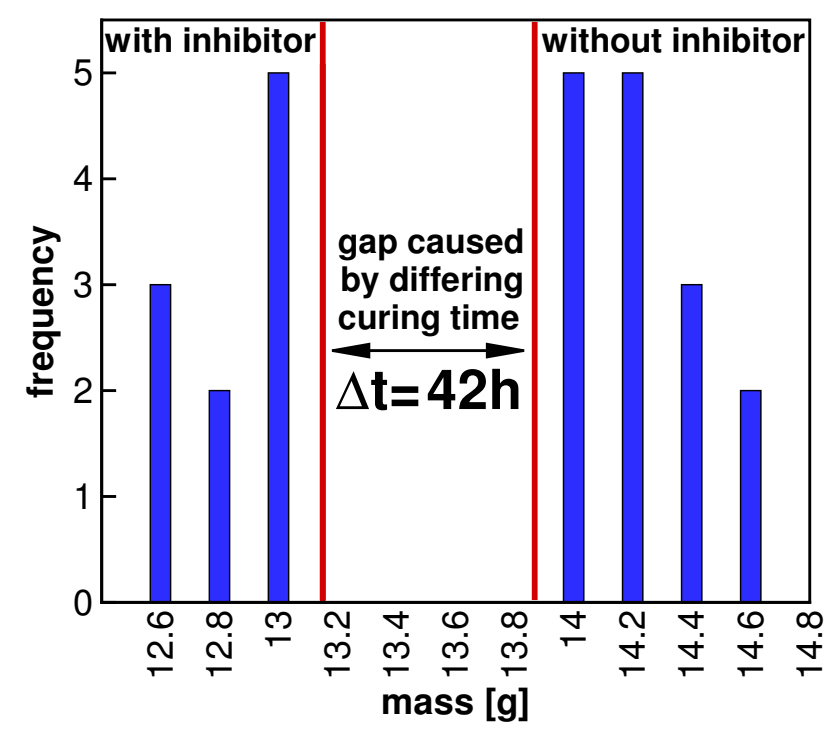

(a) Total mass distribution of produced models.

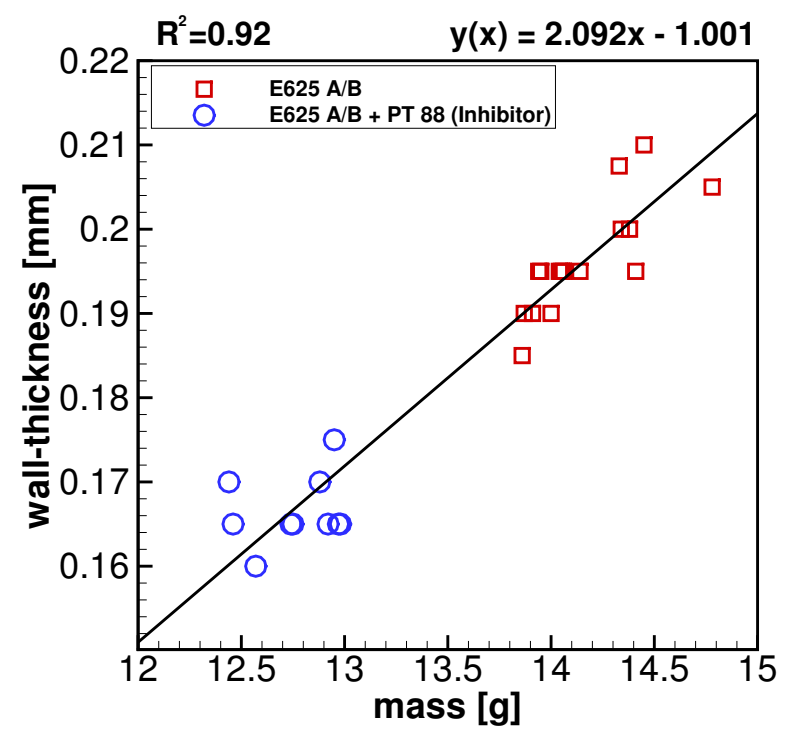

(b) Correlation between total mass and wall-thickness.

Figure A.23: Statistical evaluation of the casting process including 26 models of the flexible hemisphere.

put onto the rigid hemisphere acting as a reference for the profile measurement. Each circular hole was measured at the apex of the rigid support hemisphere to achieve minimum errors as shown in Fig. A.24(b). The results of the measurements with the laser profile scanner are depicted in Fig. A.25. Third, the actually used membrane was additionally measured by a non-contact one-sided thickness measurement (Micro-Epsilon) device based on an optical confocal chromatic measurement principle splitting white light into different spectra by several lenses. The measured data were in very good agreement with the laser profile scanner.

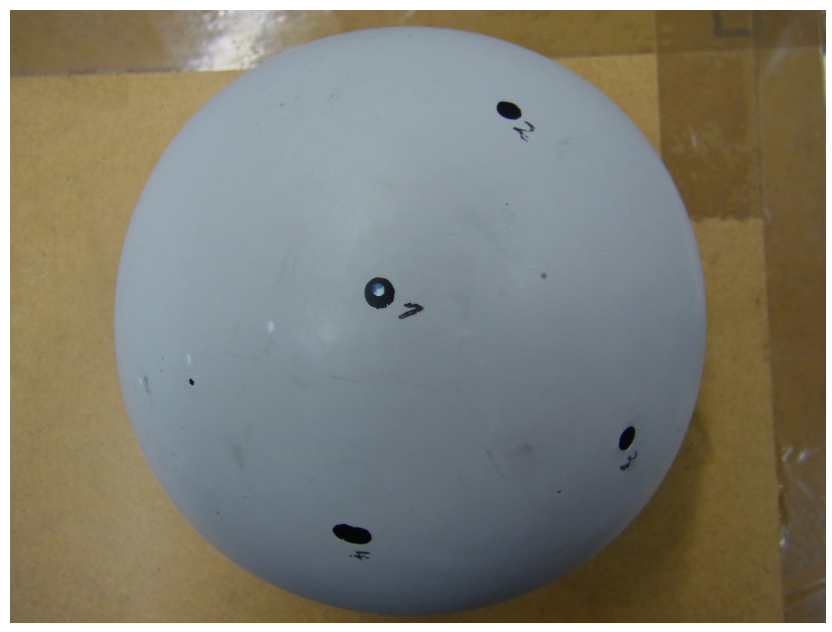

(a) 7 arbitrary holes in the hemisphere.

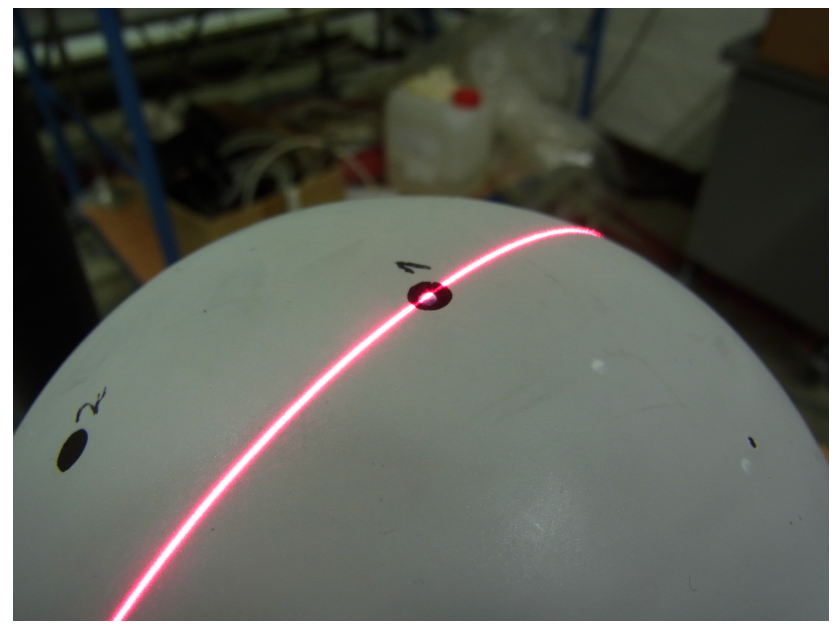

(b) Actual measurement of hole "1".

Figure A.24: Measurement of the thickness of the flexible membrane applying a high-resolution laser profile scanner.

\section{Appendix A.2. Calibration and static behavior of the flexible model}

Before the wind tunnel studies are conducted, the quality of the flexible hemisphere is evaluated. This is necessary to identify differences between the rigid and the flexible model which have to be considered when comparing the flow field results. The shape and the surface roughness are important factors that significantly influence the flow field. To characterize the differences 


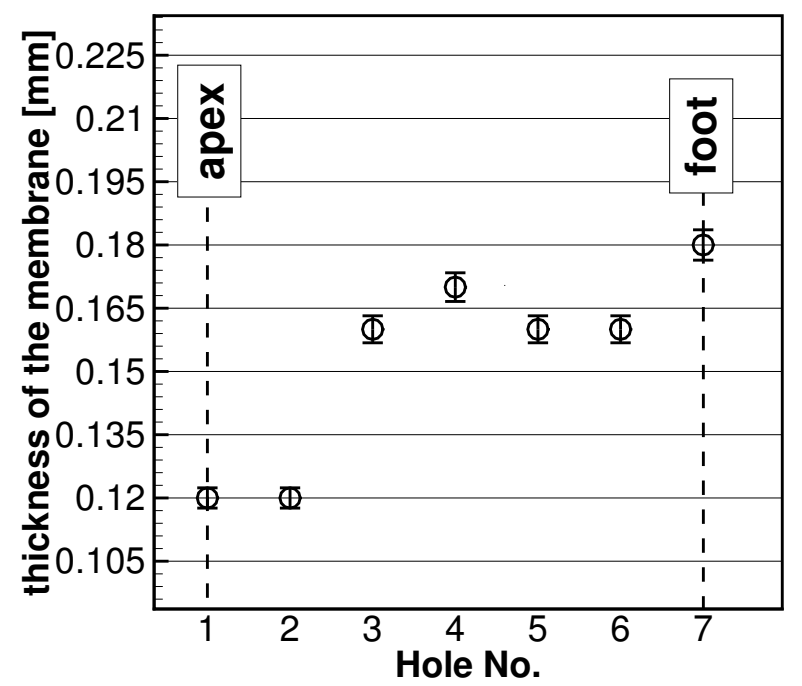

Figure A.25: Measured thickness of the membrane at each circular hole including the error of the measurements (linearity specified with $0.2 \%$ )

between both models, a preliminary calibration test is conducted outside the wind tunnel. A special setup is presented in Fig. A.26 used for the calibration procedure.

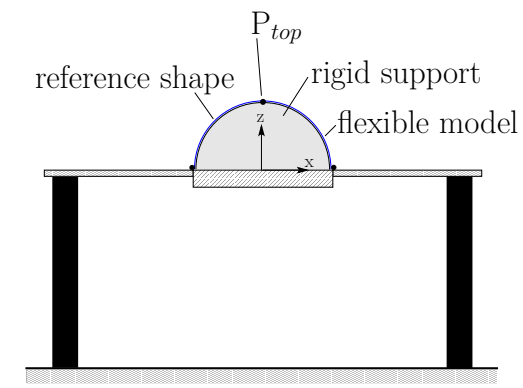

(a) Reference case: Rigid support.
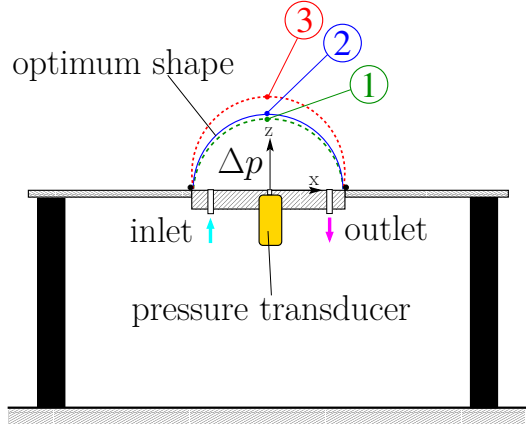

(b) Step-wise pressurization.

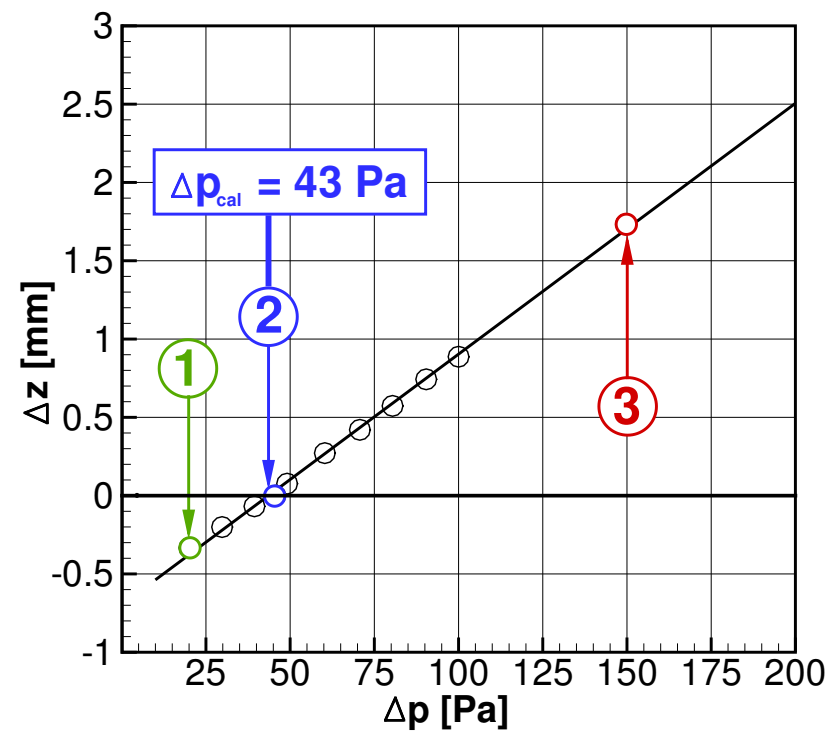

(c) Calibration chart of the applied model.

Figure A.26: Calibration test to characterize the differences between the pressurized flexible hemisphere and the rigid reference.

In a first step (Fig. A.26(a)) a solid hemisphere is used as rigid support. Its diameter is identical to the positive form of the casting process $\left(D_{\text {pos }}=149.6 \mathrm{~mm}\right)$. The flexible model is put onto the rigid support. After covering the solid form, the overall diameter is raised to about $D \approx 150 \mathrm{~mm}$ due to the wall thickness of the silicone model. This procedure ensures that the wind tunnel models of the rigid and the flexible hemisphere have nearly identical 
extensions. In this setup the supported flexible model is not affected by the inner pressure or gravitational effects and is thus used as the reference configuration which represents the rigid case best possible. A measurement by the digital-image correlation (DIC) technique is applied to determine the surface shape of the reference configuration $\Omega_{0}$.

In a second step, the rigid support is removed. A flat circular sealing with installed pressure connection, including a pressure transducer, is fixed to the lower side of the table. Then, the flexible hemisphere is pressurized in order to restore its hemispherical shape as depicted in Fig. A.26(b). The applied silicone tends to shrink about $0.1 \%$ which leads to a reduction of the initial diameter of the flexible model. This effect and the gravitational acceleration are compensated by applying a certain pressure difference compared to the surrounding leading to a best fit configuration, further on denoted as calibration configuration $\Omega_{\text {cal }}$.

The actual calibration procedure includes a step-wise pressurization of the flexible hemisphere in the range $15 \mathrm{~Pa} \leq \Delta p \leq 300 \mathrm{~Pa}$. For the calibration process a monitoring point $\mathrm{P}_{\text {top }}(0,0, D / 2)$ at the apex of the hemispherical dome is chosen to track the displacements $\Delta z$. It is assumed that the point $\mathrm{P}_{\text {top }}$ is mainly moving in vertical direction due to the rotational symmetry of the hemisphere. As the flexible structure expands with increasing pressure difference $\Delta p$, the displacement of the monitoring point is measured, schematically indicated in Fig. A.26(b) by the spots (1), (2) and (3). It is expected that with increasing pressure difference the hemisphere reaches a certain configuration $\Omega_{i}$ (represented by spot (2)), where the inflated shape matches optimally the reference shape, i.e., $\Omega_{0}-\Omega_{i} \approx 0$. This optimum is found in the calibration diagram in Fig. A.26(c). At a pressure difference of $\Delta p_{\text {cal }}=43 \mathrm{~Pa}$ the displacement $\Delta z$ is nearly zero and thus this calibration configuration $\Omega_{\text {cal }}$ has a minimum deviation from the supported reference configuration. Additionally, spot (3) indicates that the relationship between the applied gauge pressure and the resulting displacement can be assumed to be linear in the investigated range.

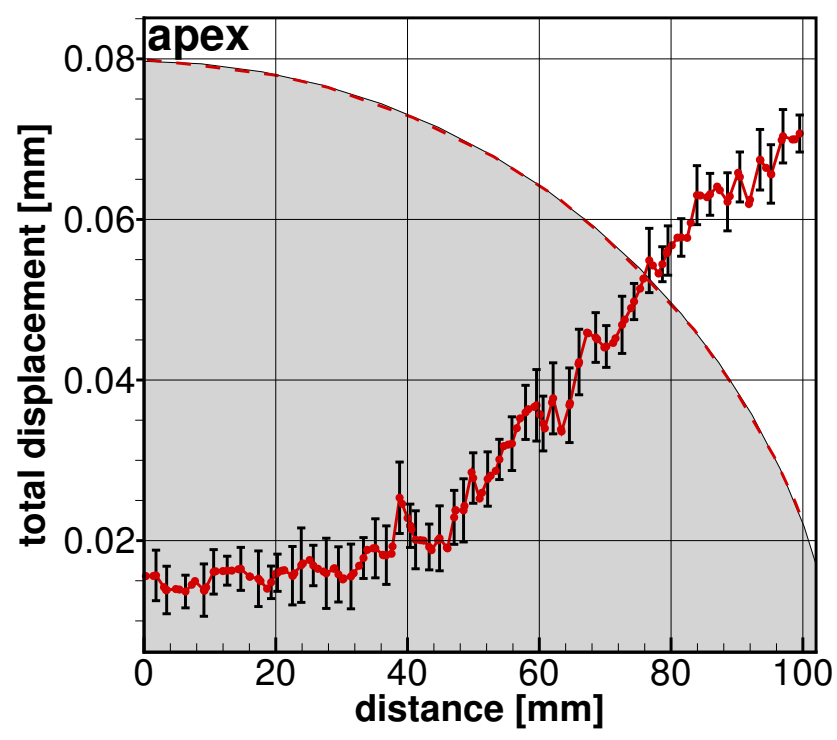

Figure A.27: Comparison of the rigidly supported hemisphere (reference configuration) with the flexible hemisphere under an inner pressure difference $\Delta p_{\text {ref }}=43 \mathrm{~Pa}$.

To characterize the shape matching in more detail, a surface line element is used to show the difference between the supported reference case and the inflated flexible hemisphere at $\Delta p_{\text {cal }}=43 \mathrm{~Pa}$. Figure A.27 presents the total displacements $\Delta r=\sqrt{\Delta x^{2}+\Delta y^{2}+\Delta z^{2}}$ (in mm) along the distance (arc length) of the applied line element (red dashed line). The maximum 
deviation between the reference and the calibrated configuration is about $\left.\Delta r\right|_{\max }=0.07 \mathrm{~mm}$. This corresponds to a maximum error of $e_{\max }=\Delta r_{\max } /\left(D_{\text {ref }} / 2\right)=0.0009(0.09 \%)$.

Furthermore, the surface measurement can be used to evaluate the arithmetic average surface roughness $R_{a}$ of the flexible model. It can be calculated as $R_{a}=(1 / n) \sum_{i=1}^{n}\left|\Delta r_{i}-\Delta \bar{r}\right|$, where $\Delta r_{i}$ is the total displacement at the location $i$ and $\Delta \bar{r}$ is the mean total displacement. For the presented line element the arithmetic average surface roughness is estimated to $R_{a}=16 \mu \mathrm{m}$. This is significantly higher than in the rigid case $\left(R_{a}=0.8 \mu \mathrm{m}\right.$, Wood et al. (2016)) but still considered to be smooth since the surface variations are within the viscous sublayer of the boundary layer leading to a maximum value of $z^{+} \approx 0.46$ at $\operatorname{Re}=100,000$. The surface roughness of the silicone model is mainly caused by unavoidable small deformations due to the casting process, micro-bubble inclusions, shrinking effects and the applied speckle pattern.

\section{Appendix A.3. Pressure settings applied for fluid-structure interaction investigations}

The intended deformations of the flexible hemisphere during the wind tunnel test shall be mainly driven by the difference between the outer pressure distribution acting on the surface of the membrane and the gauge pressure that stabilizes the model. Large quasi-static global deflections (wrinkling, buckling) due to a high pressure difference between the environment and the interior of the model have to be avoided to maintain geometrical comparability between the rigid and the flexible model. Rather the membrane should be excited by the turbulent fluid flow. Consequently, the pre-stress originating from the pressure difference should be small, since the inner pressure is primarily used to stabilize the hemispherical shape. A preliminary wind tunnel study has shown that the deformations of the flexible structure are hardly observable when applying the calibration configuration $\left(\Delta p_{\text {cal }}=43 \mathrm{~Pa}\right)$ at wind velocities $U_{\infty}<10.24 \mathrm{~m} / \mathrm{s}$ $(\operatorname{Re}<100,000)$. This is mainly caused by the stress distribution within the membrane plane which increases the stiffness of the model and makes it less vulnerable to wind loads. In order to generate significant deformations at lower wind loads $(\operatorname{Re}<100,000)$ the pressure inside the flexible model has to be adjusted accordingly. Therefore, the operating pressure value to receive the desired deformations is evaluated experimentally at each wind velocity. This procedure leads to the operating gauge pressures of $\Delta p_{\mathrm{FSI}_{1}}=19 \mathrm{~Pa}$ at $\mathrm{Re}=50,000, \Delta p_{\mathrm{FSI}_{2}}=34 \mathrm{~Pa}$ at $\mathrm{Re}=75,000$ and $\Delta p_{\mathrm{FSI}_{3}}=43 \mathrm{~Pa}$ at $\mathrm{Re}=100.000$. Below these values the inner pressure is not able to withstand the wind loads and large global deformations at the stagnation area in front of the hemisphere arise. As a consequence according to the calibration diagram (Fig. A.26(c), spot (1)) the overall maximum shape error increases to $e_{\max }=0.56 \%$ at a Reynolds number of $\operatorname{Re}=50,000$.

\section{Appendix A.4. Independency between the natural frequencies and the gauge pressure}

A preliminary study concerning the dependency of the natural frequency response of the flexible structure from different gauge pressures $(\Delta p=50 \mathrm{~Pa}, 100 \mathrm{~Pa}$ and $200 \mathrm{~Pa})$ was carried out. For this purpose, the DIC measurement system (at $1000 \mathrm{fps}$ ) was used to track the displacements of the apex of the flexible structure. An impact was applied at the lateral side of the hemisphere strongly exciting the second natural frequency $f_{n}^{2} \approx 43 \mathrm{~Pa}$. This is visible in Fig. A.28 indicating no significant influence of the chosen gauge pressure on the frequency response of the hemisphere.

\section{Appendix A.5. Connection to the test section}

A schematic illustration of the installation of the model on the flat plate is depicted in Fig. A.29(a). The cylindrical extension of the hemisphere is put into a circular cavity that was milled into the flat plate (Fig. A.29(c)). 


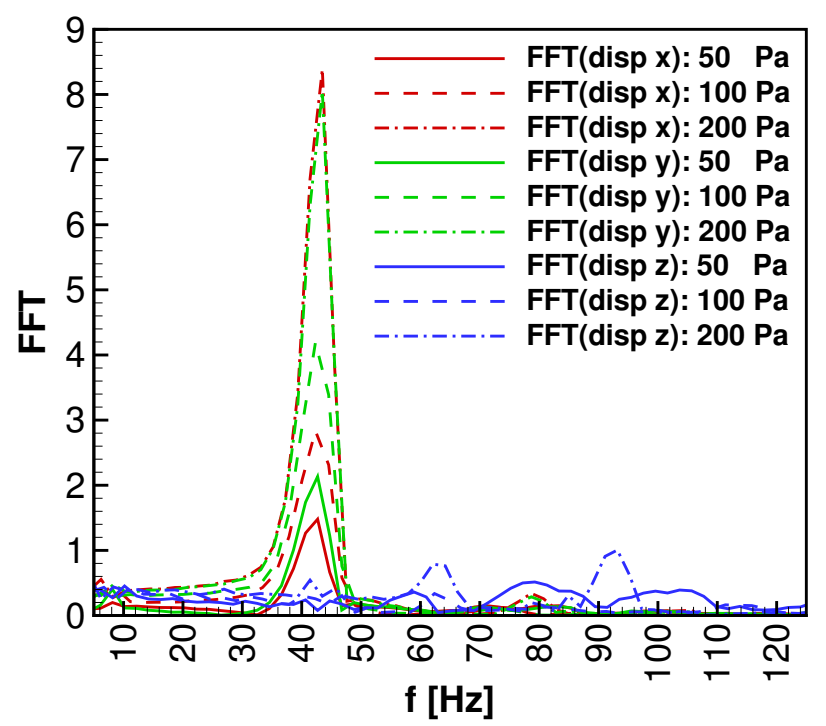

Figure A.28: Frequency response of the flexible hemisphere at the apex measured at different gauge pressures.

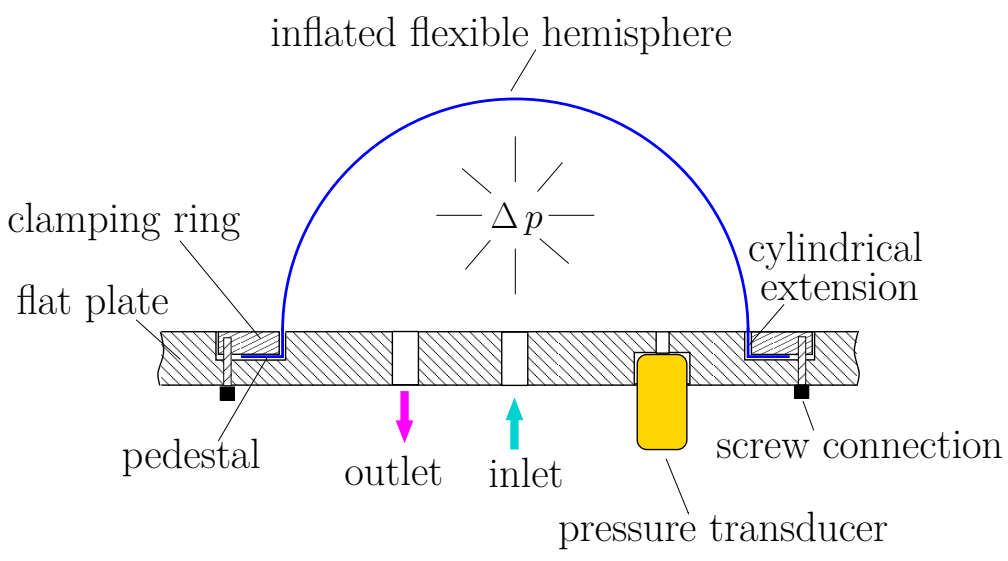

(a) Connection of the flexible hemisphere and the flat plate.

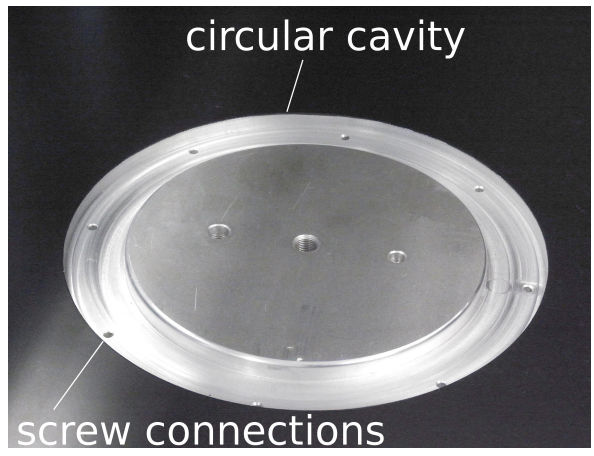

(b) Circular cavity on the flat plate.

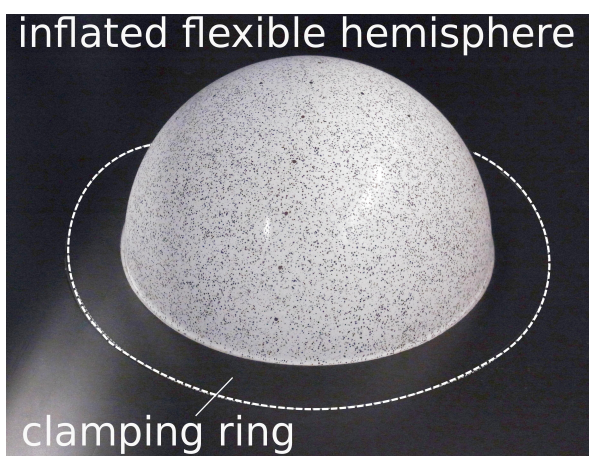

(c) Actual setup of the flexible model.

Figure A.29: Sketch of the installation of the flexible hemisphere on the flat plate.

A corresponding clamping ring is placed into the cavity and presses onto the pedestal of the model. The connection is fixed by stud hole screws. To maintain an even surface of the flat plate, the height of the ring can be adjusted by the screws on the lower side. Furthermore, air inlet and outlet drillings ensure a constant pressurization inside the hemisphere in order to avoid air-leakage effects. The actual setup of the inflated model inside the test section of the wind tunnel is depicted in Fig. A.29(c). 


\section{Appendix B. Material properties}

\section{Appendix B.1. Young's modulus and Poisson ratio of the silicone}

The material properties of the silicone were determined to characterize the structural behavior of the utilized model (Fig. B.30). A standardized plastic tensile test (EN ISO 527-2, ASTM D638) was applied in order to receive the strain-stress correlation of the material with a standardized specimen (Fig. B.30(c)). The measured data in Fig. B.30(a) show a non-linear behavior which is typical for rubber-type materials. At low strain rates the curve indicates a nearly linear behavior in the range $0 \leq \varepsilon \leq 0.1$, which can be approximated by a constant Young's modulus. The slope in this particular region can be evaluated by a linear regression (black line in the diagram) which leads to an elastic modulus of the silicone of about $E_{\text {silicone }}=7 \times 10^{5} \mathrm{~Pa}$. Furthermore, the levels of the maximum principal Lagrange strain reached during the measurements of the flexible hemisphere at $\mathrm{Re}=100,000$ are determined. The maximum principal Lagrange strain is calculated in several steps. First, the theoretical components of the Lagrange strain are computed based on the local gradients containing the displacement in $x$-direction $\Phi_{x}$ and in $y$-direction $\Phi_{y}$ following Bonet and Wood (1997):

$$
\begin{gathered}
\varepsilon_{x x}=\frac{\partial \Phi_{x}}{\partial x}+\frac{1}{2}\left[\left(\frac{\partial \Phi_{x}}{\partial x}\right)^{2}+\left(\frac{\partial \Phi_{y}}{\partial x}\right)^{2}\right] \\
\varepsilon_{y y}=\frac{\partial \Phi_{y}}{\partial y}+\frac{1}{2}\left[\left(\frac{\partial \Phi_{x}}{\partial y}\right)^{2}+\left(\frac{\partial \Phi_{y}}{\partial y}\right)^{2}\right] \\
\varepsilon_{x y}=\frac{1}{2}\left(\frac{\partial \Phi_{x}}{\partial y}+\frac{\partial \Phi_{y}}{\partial x}\right)+\frac{1}{2}\left(\frac{\partial \Phi_{x}}{\partial x} \frac{\partial \Phi_{x}}{\partial y}+\frac{\partial \Phi_{y}}{\partial x} \frac{\partial \Phi_{y}}{\partial y}\right)
\end{gathered}
$$

The basis of the resulting Lagrange strain matrix $\mathbb{E}$ is then changed to the basis of the principal strain directions. The shear strain components in this basis are zero. It is equivalent to solve the eigenvalue problem associated to the matrix $\mathbb{E}$, where these eigenvalues are the principal strains. As the applied software is restricted to the $x$ - and $y$-component, both principal strain values are given by:

$$
\varepsilon_{1}, \varepsilon_{2}=\frac{\varepsilon_{x x}+\varepsilon_{y y}}{2} \pm \sqrt{\left(\frac{\varepsilon_{x x}-\varepsilon_{y y}}{2}\right)^{2}+\left(\varepsilon_{x y}\right)^{2}}
$$

with $\varepsilon_{1}=\varepsilon_{\max }$ and $\varepsilon_{2}=\varepsilon_{\min }$.

Note that due to the small displacements of the present cases the measured Lagrange strain components converge to the measured components of the simpler engineering strain, i.e., the local displacement gradient. As no visual difference can be made between both, plots containing the engineering strains are not added.

The strain values are depicted in Fig. B.30(d) indicating an average value of the maximum principal Lagrange strain of $\varepsilon_{1}^{a v g}=0.045$. An overall distribution of the total displacements and corresponding maximum principal Lagrange strain at an arbitrary time step are depicted in Figs. B.30(e) and (f).

Additionally, the levels of the maximum principal Lagrange strain during the static inflation test without wind loads are presented in Fig. B.31. The inflation procedure followed a step-wise 
pressurization of the flexible structure. Figure B.31(c) depicts the deformation in $z$-direction at the apex of the flexible hemisphere, whereas the corresponding maximum principal Lagrange strains are shown in Fig. B.31(d). Note that the reference for the calculation of the strain is the already pre-stressed configuration $\Omega_{\text {cal }}$ at $\Delta p_{\text {cal }}=43 \mathrm{~Pa}$. However, also this initial stretching by the reference gauge pressure does not violate the strain limit of $\varepsilon_{1}<0.1$. Thus, the linearization of the Young's modulus is valid.

\section{Appendix B.2. Damping characteristics}

Additionally, the damping ratio of the silicone was determined to about $b_{\text {material }}=2.5 \%$ with a standardized pendulum test (EN ISO 6721-2) at room temperature depicted in Fig. B.32. The evaluation procedure is as follows. By using the logarithmic decrement

$$
\Lambda=\ln \frac{d\left(t_{i}\right)}{d\left(t_{i+1}\right)}=\frac{1}{n} \ln \frac{d\left(t_{i}\right)}{d\left(t_{i+n}\right)}, \quad n=1,2, \ldots
$$

describing the ratio between the amplitudes of two chosen peak values at different points in time, the relation to the decay coefficient is as follows

$$
\delta=\omega_{d} b=\frac{\Lambda}{T_{d}}
$$

with $\omega_{d}=2 \pi f$ describing the angular frequency of the damped system, $b$ the damping ratio and $T_{d}$ the period between two peaks of the damped oscillation.

$$
b=\frac{\Lambda}{\omega_{d} T_{d}}=\frac{\Lambda}{2 \pi f \frac{1}{f}}=\frac{\Lambda}{2 \pi}
$$

The data obtained by the pendulum measurements are depicted in Figs. B.32(a) - (c). The logarithmic decrement was calculated by setting the first peak into relation to the 10th peak according to equation (B.5). Afterwards, the damping ratio $b$ can be easily calculated by equation (B.7). The values for the pendulum test are listed in Table B.5.

Table B.5: Damping ratio and logarithmic decrement determined by the pendulum test.

\begin{tabular}{|c|c|c|}
\hline Specimen & log. decrement $\Lambda$ & damping ratio $b$ \\
\hline $\mathbf{1}$ & 0.153 & 0.024 \\
\hline $\mathbf{2}$ & 0.156 & 0.025 \\
\hline $\mathbf{3}$ & 0.134 & 0.026 \\
\hline
\end{tabular}

However, the pendulum test does not include other damping effects that are present in the actual setup of the air-inflated hemisphere. In this case, the gauge pressure is also acting as a damping source. To estimate this, the dynamic response test is used to receive a value of the damping of the entire system including the silicone membrane and the gauge pressure. For this purpose, the same approach as above is applied to the data shown in Figs. B.33(b) and (c) . The data were generated based on the flexible hemisphere applied in the wind tunnel setup and pressurized with a gauge pressure of $\Delta p_{\text {cal }}=43 \mathrm{~Pa}$. The structure was excited by a brief impact at the lateral side of the hemisphere. The displacements of the structure were measured at the apex of the hemisphere at a resolution of $1000 \mathrm{fps}$ with the DIC system. This procedure was repeated several times to ensure reproducibility. The damping behavior of the actual system 


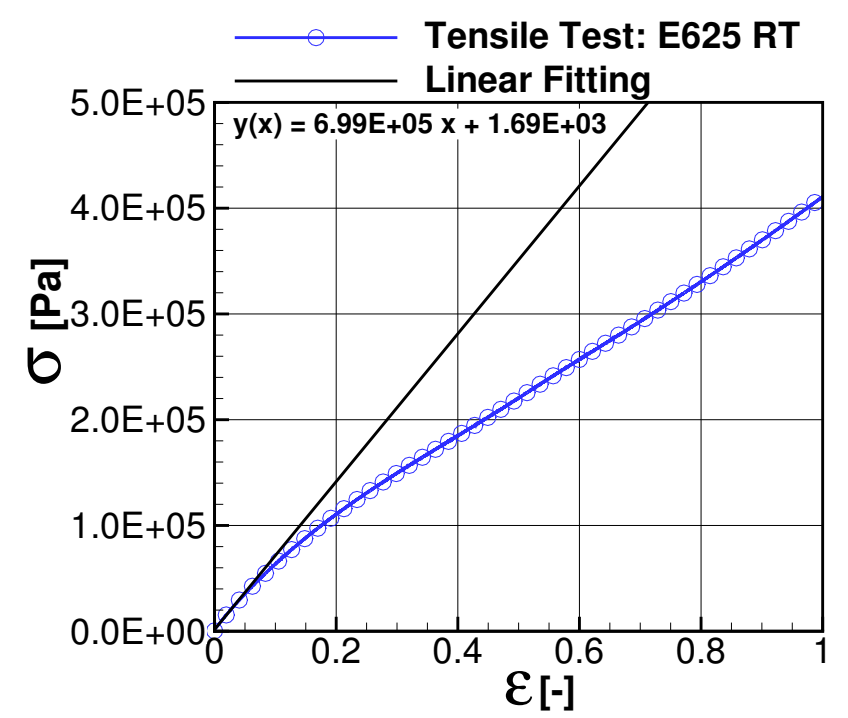

(a) Strain-stress curve of tensile test.

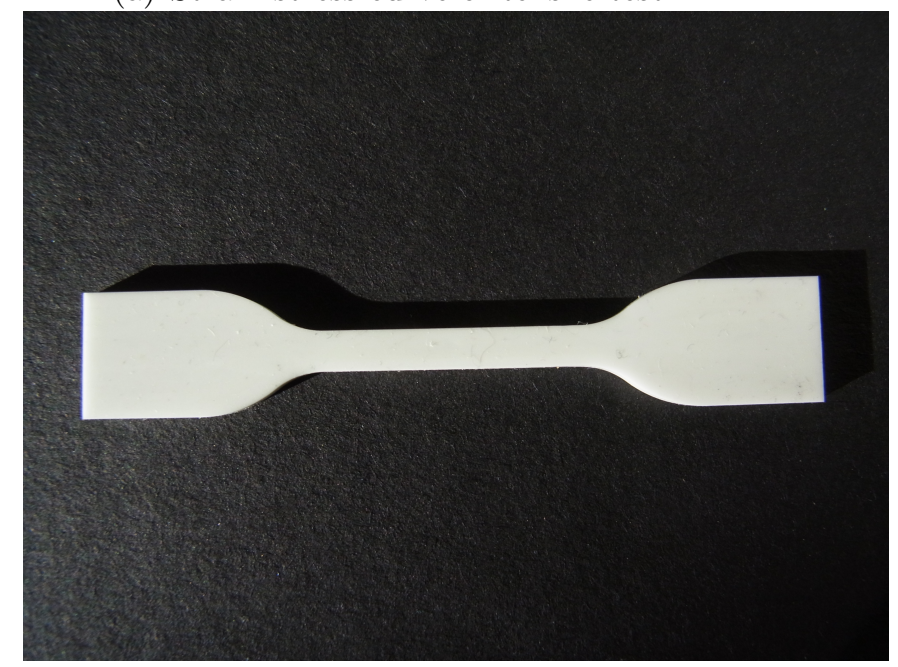

(c) Specimen used for the tensile test.

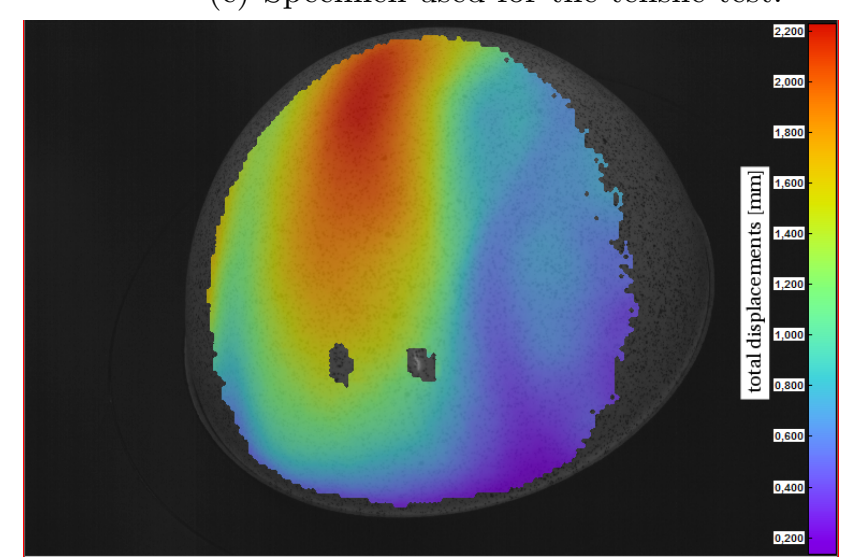

(e) Total displacements in $\mathrm{mm}$.

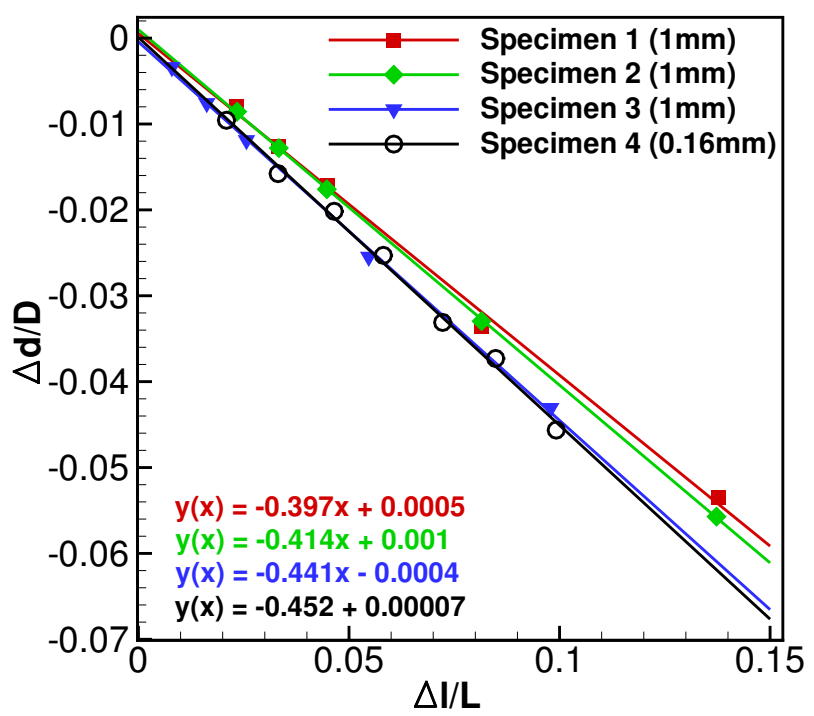

(b) Poisson ratio of the tested specimens.

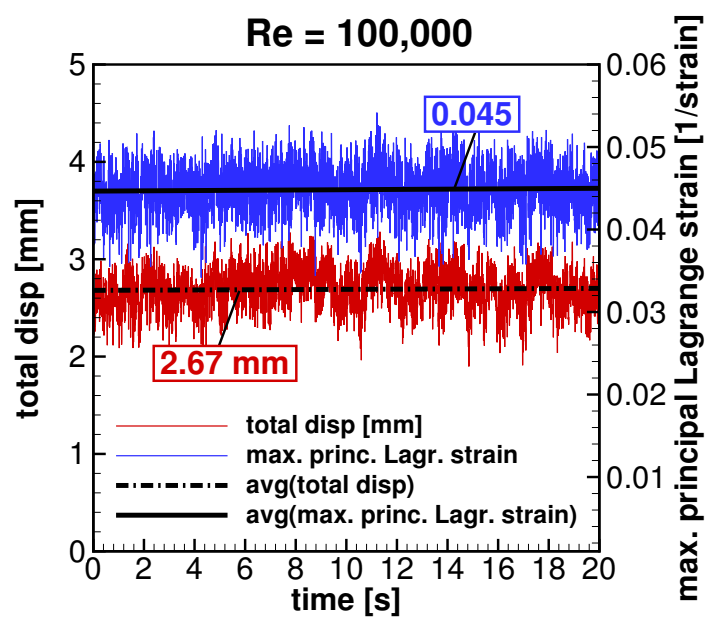

(d) Strain and disp. at apex $\operatorname{Re}=100,000$.

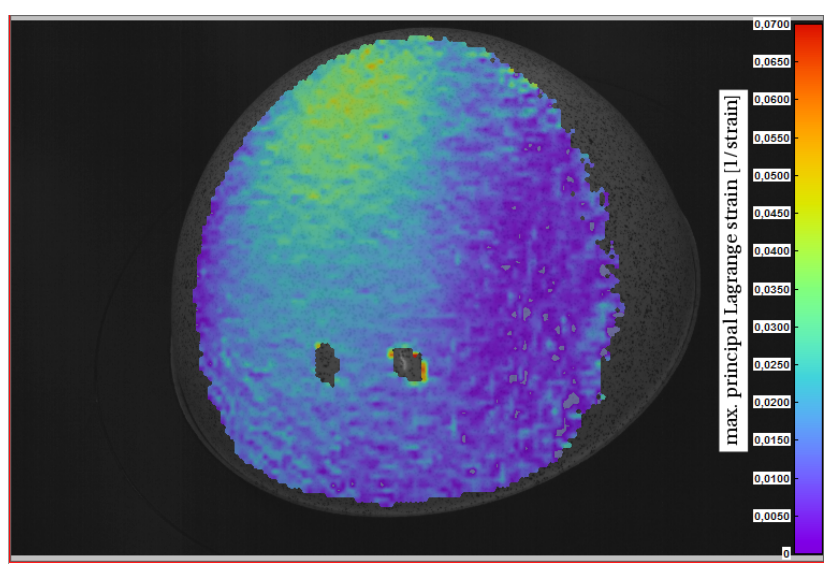

(f) Maximum principal Lagrange strain.

Figure B.30: Evaluation of the elastic material properties of the silicone Elastosil ${ }^{\circledR}$ RT 625 A/B including maximum strain levels during the wind tunnel measurements at the apex with additional arbitrary DIC data (total displacements, maximum principal Lagrange strain) of the correlation area at $\operatorname{Re}=100,000$.

including the gauge pressure differs from the pendulum test. An ideal beam under normal atmospheric conditions oscillates freely with a regular motion of the decay until it comes to 


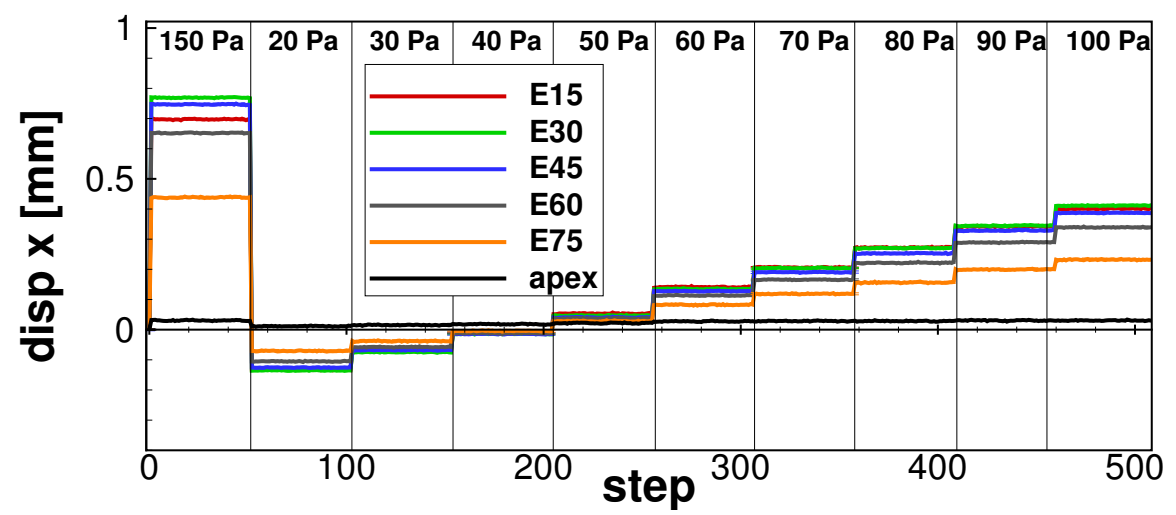

(a) $\Delta x$ displacements.

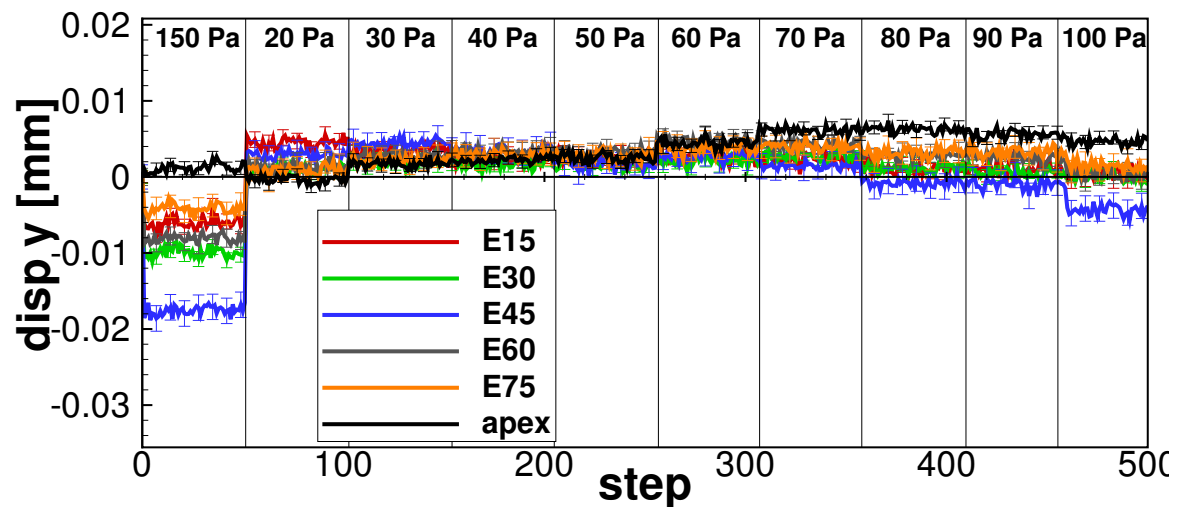

(b) $\Delta y$ displacements.

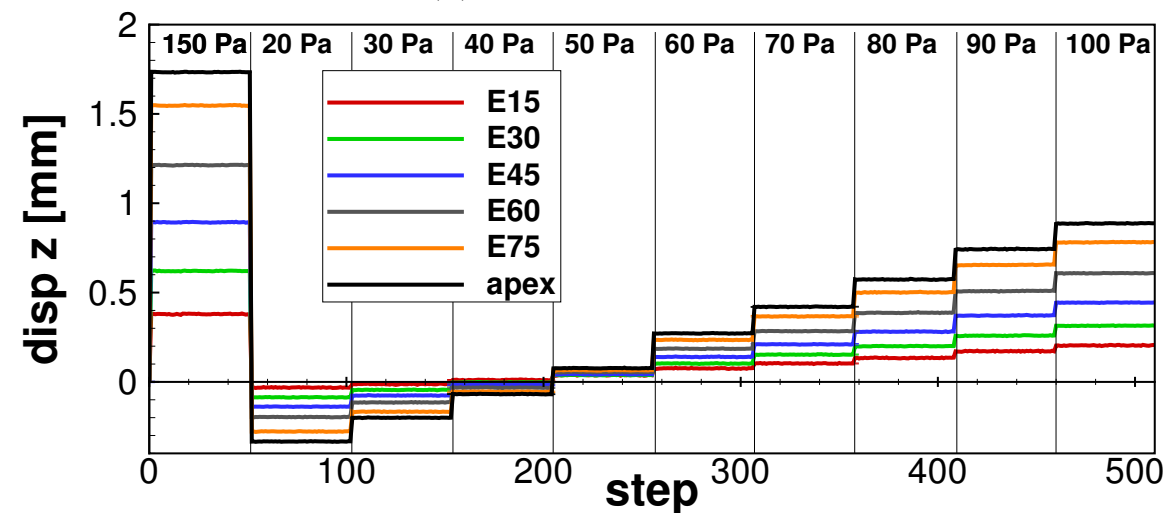

(c) $\Delta z$ displacements.

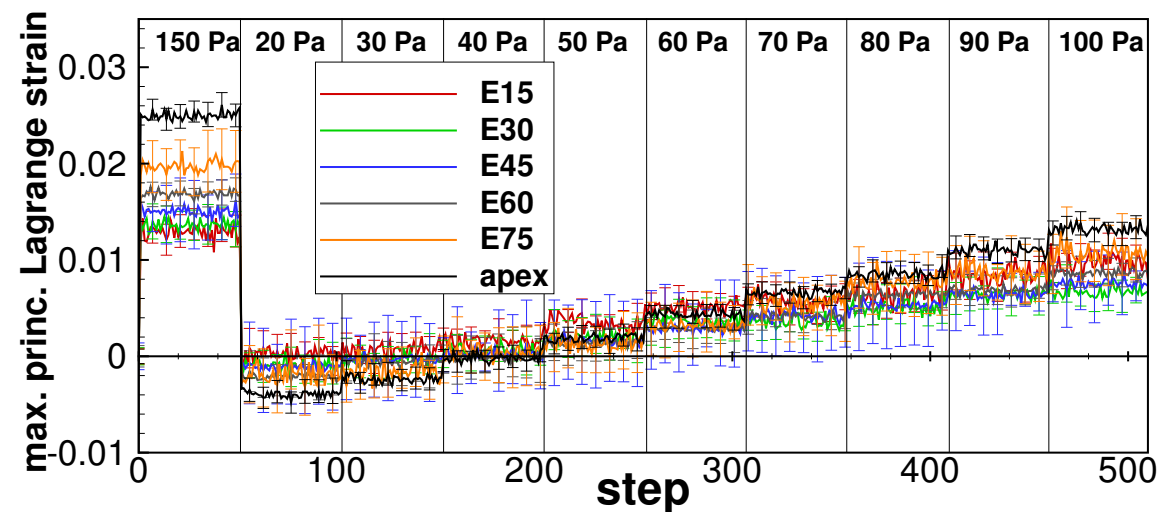

(d) Maximal principal Lagrange strain at each monitoring point.

Figure B.31: Displacement and maximum principal strain response due to a step-wise increasing gauge pressure. 


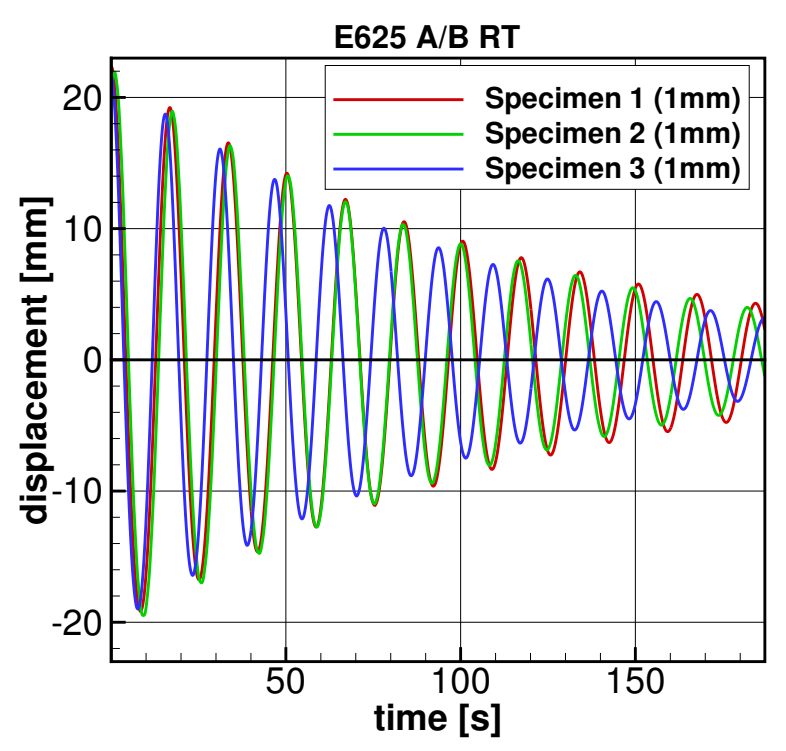

(a) Decay of motion during pendulum test.

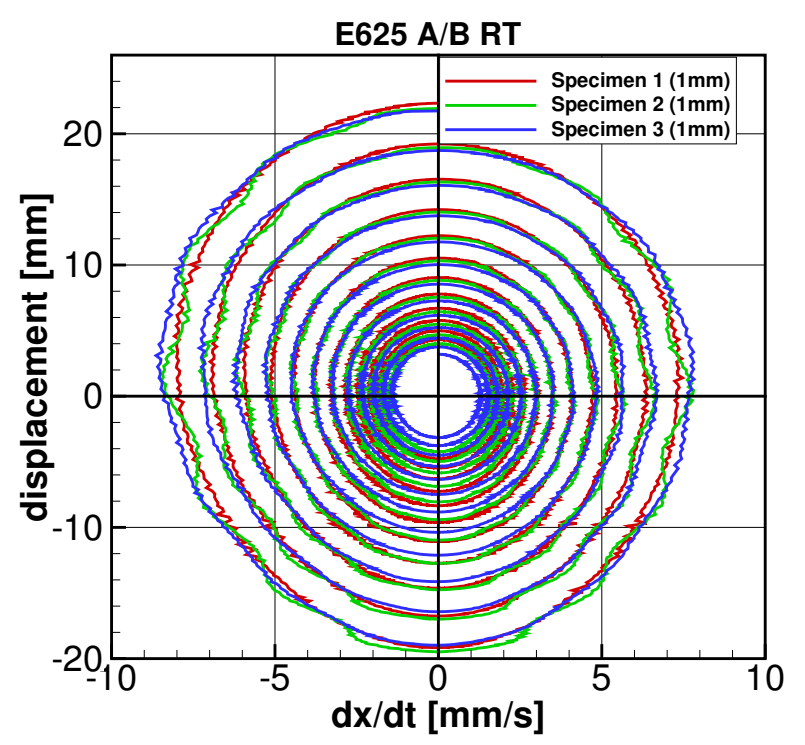

(b) Phase characteristics of each specimen.

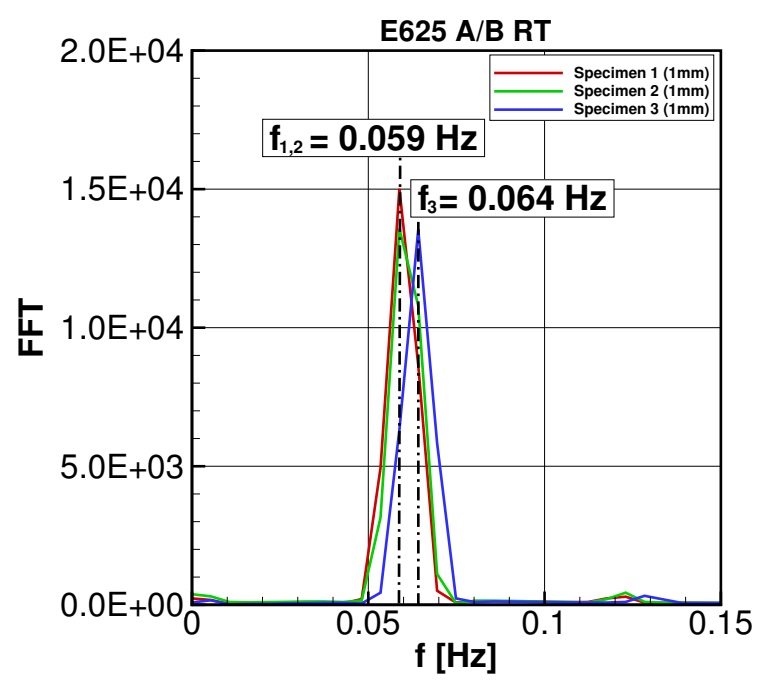

(c) Damped natural frequencies of each specimen.

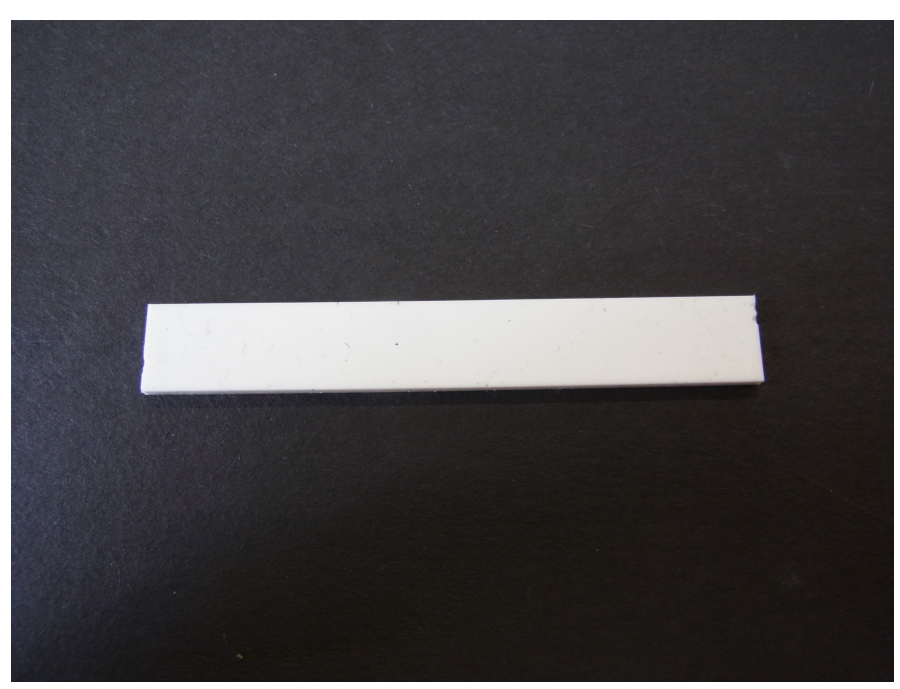

(d) Pendulum test specimen.

Figure B.32: Pendulum test measurements to evaluate the damping ratio $D$ of the applied silicone material.

rest again. The pressurized hemisphere has a slightly different behavior. The force acting on the inner surface of the hemisphere due to the gauge pressure $\Delta p$ is prohibiting a regular decay of motion. Instead, the inward motion of the oscillating structure is inhibited by the gauge pressure and the outward motion is driven additionally by this pressure force resulting in the characteristic decay. Based on these results the damping ratio of the pressurized hemisphere as a complex system was determined and found in the range between $0.046 \leq b_{\text {system }} \leq 0.062$ with an average of about $\bar{b}_{\text {system }}=0.054$. 


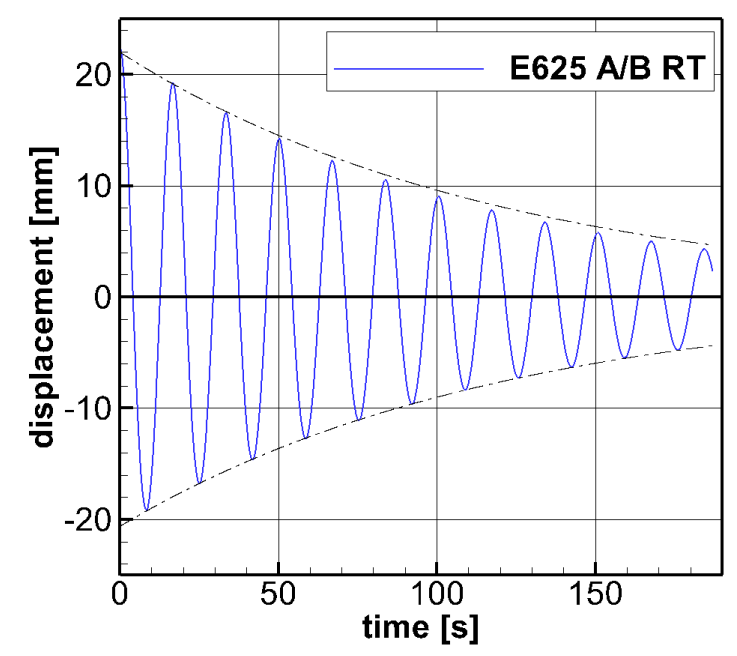

(a) Material damping (pendulum test).

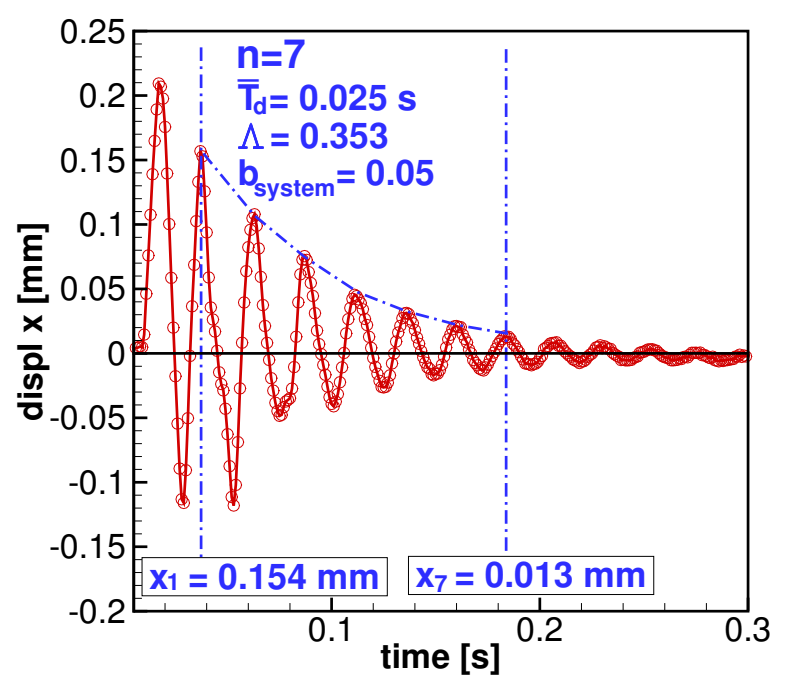

(b) Decay of motion hemisphere (apex) $\Delta x$.

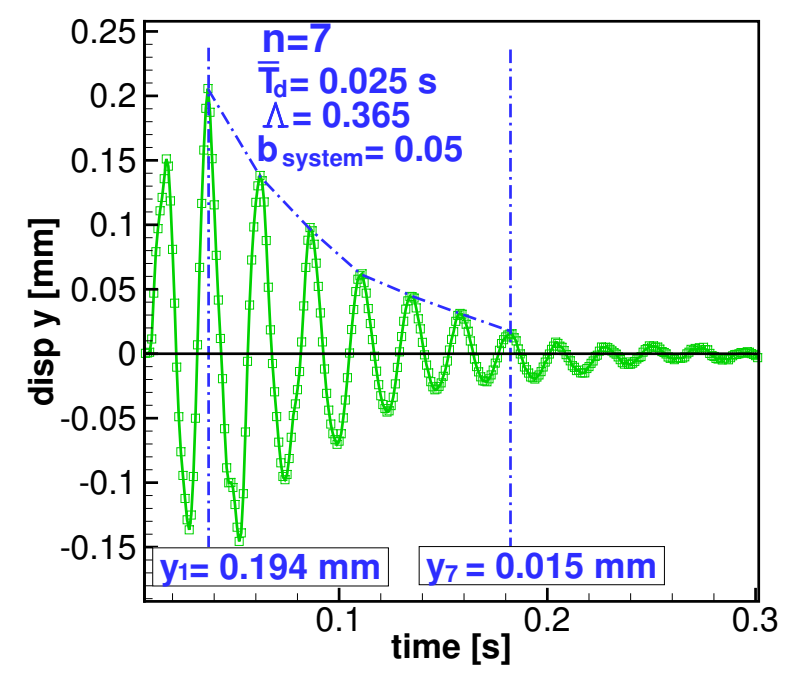

(c) Decay of motion hemisphere (apex) $\Delta y$.

Figure B.33: Characteristics of the material damping (pendulum test) and the overall damping of the system applied in the wind tunnel setup including the gauge pressure. 


\section{Appendix C. Convergence of the statistics}

A demonstration of the convergence history is plotted in Fig. C.34 for the case Re $=100,000$. The graphs in Figs. C.34(a) and (c) depict the convergence of the standard deviations $S x$ and $S z$ when increasing the number of profile lines. These plots consider the evolution of the final graph leading to a convergence at about 3500 profile lines without significant changes. Furthermore, the deltas $S j_{i}-S j_{i+500}(\mathrm{j}=x, z)$ between the standard deviations every 500 profiles are depicted in Figs. C.34(b) and (d). These plots also show a converging characteristics.

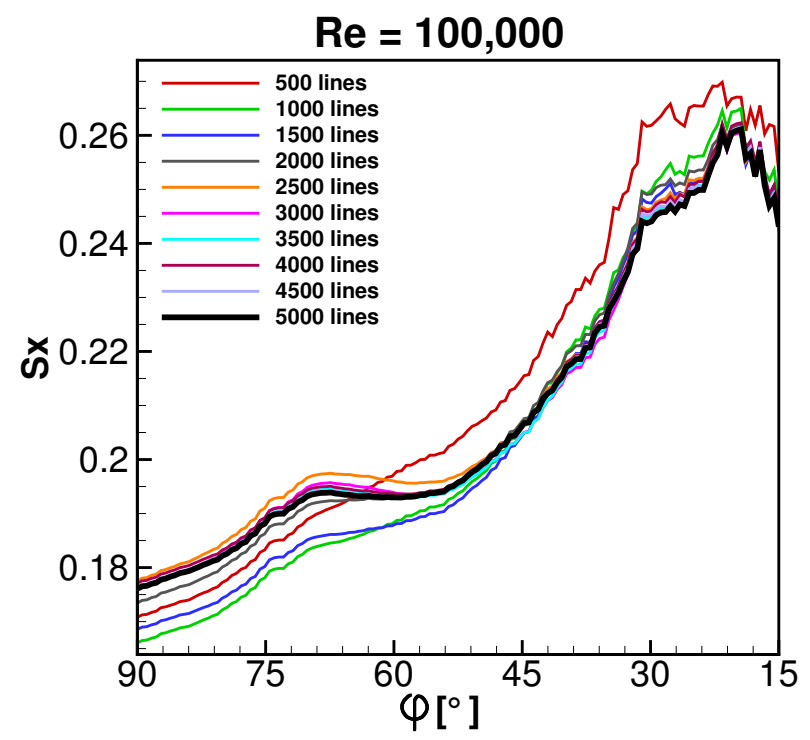

(a) Convergence of $S x$ as graph.

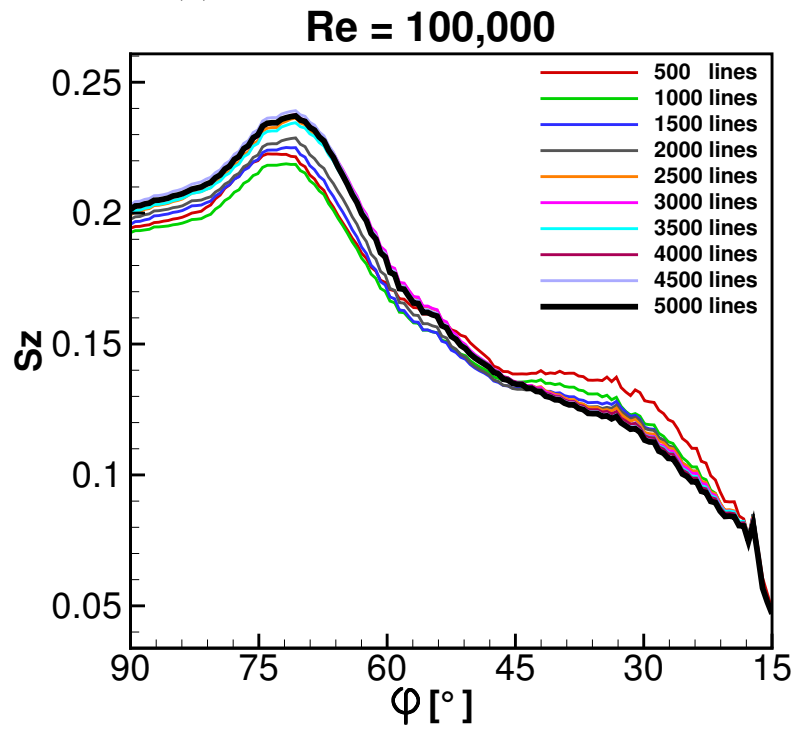

(c) Convergence of $S z$ as graph.

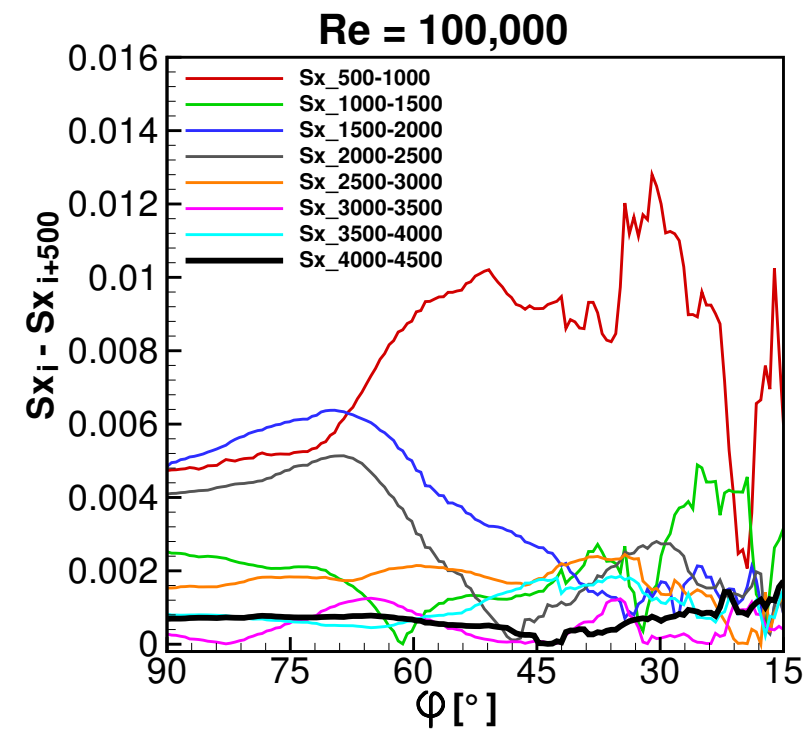

(b) $S x_{i}-S x_{i+500}$

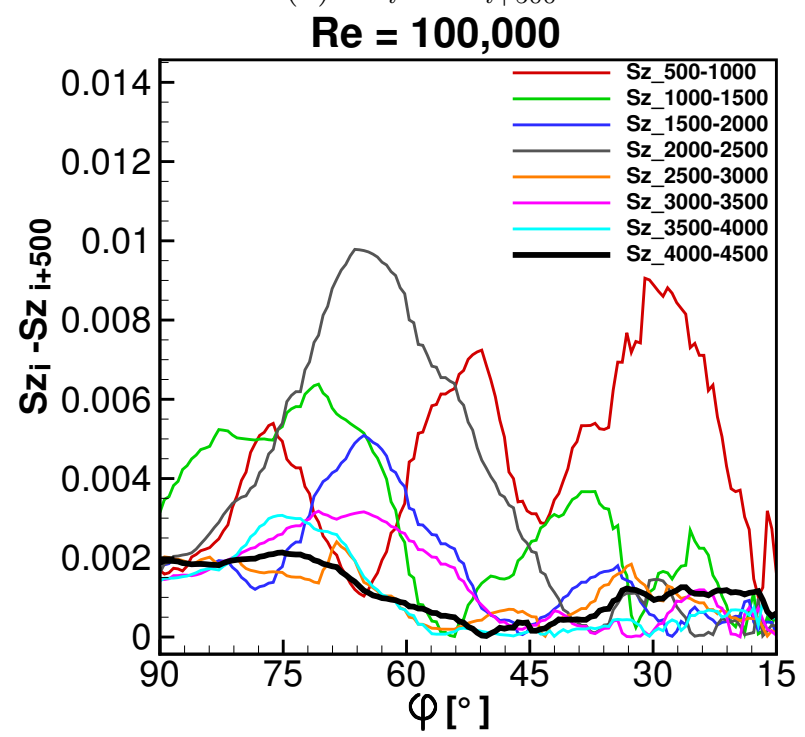

(d) $S z_{i}-S z_{i+500}$

Figure C.34: Convergence of the standard deviation $S x$ and $S z$ of the DIC measurements.

\section{References}

Abarbanel, H. D., Brown, R., Sidorowich, J. J., Tsimring, L. S., 1993. The analysis of observed chaotic data in physical systems. Reviews of modern physics 65 (4), 1331-1392.

Bonet, J., Wood, R. D., 1997. Nonlinear continuum mechanics for finite element analysis.

Cambridge University Press. 
Breuer, M., De Nayer, G., Wood, J., 2016. Complementary Experimental-Numerical Investigation of the Flow past a Rigid and Flexible Hemisphere in Turbulent Flow: Part II: Numerical Simulations. In: 24. Fachtagung "Experimentelle Strömungsmechanik", 6-8 Sept., Cottbus, Germany. Vol. 24. pp. 45.1-8.

Counihan, J., 1969. An improved method of simulating an atmospheric boundary layer in a wind tunnel. Atmospheric Environment (1967) 3 (2), 197-214.

Counihan, J., 1975. Adiabatic atmospheric boundary layers: A review and analysis of data from the period 1880-1972. Atmospheric Environment (1967) 9 (10), 871-905.

Glück, M., Breuer, M., Durst, F., Halfmann, A., Rank, E., 2003. Computation of wind-induced vibrations of flexible shells and membranous structures. Journal of Fluids and Structures 17 (5), 739-765.

Gong, J., Yang, X., Zhang, Z., Zhao, J., 2010. Theoretical analysis and experimental study of an air inflated membrane structure. Journal of Zhejiang University 11 (1), 25-33.

Grenander, U., Pollak, H., Slepian, D., 1959. The distribution of quadratic forms in normal variates: A small sample theory with applications to spectral analysis. Journal of the Society for Industrial and Applied Mathematics 7 (4), 374-401.

Kneen, P., 1990. Prestressed membrane structures the ultimate thin-walled structure. ThinWalled Structures 9 (1), 135 - 149, special Volume on Thin-Walled Structures: Developments in Theory and Practice.

Larese, A., Rossi, R., Ryzhakov, P., 2014a. Embedded approach for the FSI involving inflatable light-weight structures. In: 11th. World Congress on Computational Mechanics (WCCM XI), Barcelona.

Larese, A., Rossi, R., Wüchner, R., Al Sofi, H., Ryzhakov, P., Oñate, E., 2014b. FSI analysis of light-weight structures towards a virtual wind tunnel. In: 11th. World Congress on Computational Mechanics (WCCM XI), Barcelona.

Lavoie, P., Avallone, G., De Gregorio, F., Romano, G. P., Antonia, R. A., Jul 2007. Spatial resolution of PIV for the measurement of turbulence. Experiments in Fluids 43 (1), 39-51.

Lawson, T. V., 1968. Methods of producing velocity profiles in wind tunnels. Atmospheric Environment (1967) 2 (1), 73-76.

Ligaro, S. S., Barsotti, R., 2013. Single-layer spherical geodesic domes interacting with a network of inflatable beams. In: VI International Conference on Textile Composites and Inflatable Structures, Structural Membranes, Munich. Vol. 1. pp. 520-529.

Ligrani, P. M., Moffat, R., Kays, W. M., 1979. The thermal and hydrodynamic behavior of thick, rough-wall, turbulent boundary layers. Tech. rep., DTIC Document.

Manhart, M., 1998. Vortex shedding from a hemisphere in a turbulent boundary layer. Theoretical and Computational Fluid Dynamics 12 (1), 1-28.

Michalski, A., Gawenat, B., Gelenne, P., Haug, E., 2015. Computational wind engineering of large umbrella structures. Journal of Wind Engineering and Industrial Aerodynamics 144, 96-107. 
Michalski, A., Kermel, P., Haug, E., Löhner, R., Wüchner, R., Bletzinger, K.-U., 2011. Validation of the computational fluid-structure interaction simulation at real-scale tests of a flexible $29 \mathrm{~m}$ umbrella in natural wind flow. Journal of Wind Engineering and Industrial Aerodynamics 99 (4), 400-413.

Mishali, M., Eldar, Y. C., 2010. From theory to practice: Sub-nyquist sampling of sparse wideband analog signals. IEEE Journal of Selected Topics in Signal Processing 4 (2), 375-391.

Morison, J., Johnson, J., Schaaf, S., 1950. The force exerted by surface waves on piles. Journal of Petroleum Technology 2 (5), 149-154.

Naudascher, E., Rockwell, D., 1994. Flow-induced Vibrations: An Engineering Guide. AA Balkema, Rotterdam, Holland.

Newman, B., Ganguli, U., Shrivastava, S., 1984. Flow over spherical inflated buildings. Journal of Wind Engineering and Industrial Aerodynamics 17 (3), 305-327.

Nyquist, H., 2002. Certain topics in telegraph transmission theory. Proceedings of the IEEE $90(2), 280-305$.

Orton, A., 1987. The way we build now: Form, scale and technique. Taylor and Francis.

Rank, E., Halfmann, A., Glück, M., Breuer, M., Durst, F., Kaiser, U., Bergmann, D., Wagner, S., 2003. Windbelastung leichter Flächentragwerke: Numerische Simulation und Windkanalversuch. Bauingenieur 78, 501-508.

Roberts, J., Walker, G., 2003. Artificial thickening of wind tunnel boundary layers via an array of cross-flow jets. Experimental Thermal and Fluid Science 27 (5), 583-588.

Rojratsirikul, P., Wang, Z., Gursul, I., 2009. Unsteady fluid-structure interactions of membrane airfoils at low Reynolds numbers. Experiments in Fluids 46 (5), 859-872.

Rojratsirikul, P., Wang, Z., Gursul, I., 2010. Effect of pre-strain and excess length on unsteady fluid-structure interactions of membrane airfoils. Journal of Fluids and Structures 26 (3), 359-376.

Rossi, R., 2013. Advanced methods for the coupled simulation of membrane systems: Towards a virtual wind tunnel. In: VI International Conference on Textile Composites and Inflatable Structures, Structural Membranes, Munich.

Rosti, M. E., Brandt, L., 2017. Numerical simulation of turbulent channel flow over a viscous hyper-elastic wall. Journal of Fluid Mechanics 830, 708-735.

Sargison, J. E., Walker, G. J., Bond, V., Chevalier, G., December 2004. Experimental review of devices to artificially thicken wind tunnel boundary layers. In: Behnia, M., Lin, W., McBain, G. D. (Eds.), Proceedings of the Fifteenth Australasian Fluid Mechanics Conference (CD-ROM). The University of Sydney, Sydney, Australia, AFMC00091.

Shen, L., Zhang, X., Yue, D. K. P., Triantafyllou, M. S., 2003. Turbulent flow over a flexible wall undergoing a streamwise travelling wave motion. Journal of Fluid Mechanics 484, 197221.

Tamai, N., Asaeda, T., Tanaka, N., 1987. Vortex structures around a hemispheric hump. Boundary-Layer Meteorology 39 (3), 301-314. 
Tavakol, M. M., Abouali, O., Yaghoubi, M., 2015. Large eddy simulation of turbulent flow around a wall mounted hemisphere. Applied Mathematical Modelling 39 (13), 3596-3618.

Wood, J. N., Breuer, M., 2017. Studies on the Fluid-Structure Interaction of a Pressurized Membranous Hemisphere in Turbulent Flow. In: 25. Fachtagung "Experimentelle Strömungsmechanik", 5-7 Sept., Karlsruhe, Germany. Vol. 25. pp. 81-88.

Wood, J. N., De Nayer, G., Schmidt, S., Breuer, M., 2016. Experimental investigation and large-eddy simulation of the turbulent flow past a smooth and rigid hemisphere. J. Flow, Turbulence and Combustion 97 (1), 79-119.

Wu, Y., Chen, Z.-q., Sun, X.-y., 2015. Research on the wind-induced aero-elastic response of closed-type saddle-shaped tensioned membrane models. Journal of Zhejiang University 16 (8), 656-668. 